\title{
The Weyl BMS group and Einstein's equations
}

\author{
Laurent Freidel, ${ }^{a}$ Roberto Oliveri, $^{b}$ Daniele Pranzetti ${ }^{a, c}$ and Simone Speziale ${ }^{d}$ \\ ${ }^{a}$ Perimeter Institute for Theoretical Physics, \\ 31 Caroline Street North, Waterloo, Ontario N2L 2Y5, Canada \\ ${ }^{b}$ CEICO, Institute of Physics of the Czech Academy of Sciences, \\ Na Slovance 2, 182 21 Praha 8, Czech Republic \\ ${ }^{c}$ Università degli Studi di Udine, \\ via Palladio 8, I-33100 Udine, Italy \\ ${ }^{d}$ CPT-UMR 7332, \\ CNRS, 13288 Marseille, France \\ E-mail: lfreidel@perimeterinstitute.ca, roliveri@fzu.cz, \\ dpranzetti@perimeterinstitute.ca, simone.speziale@cpt.univ-mrs.fr
}

ABSTRACT: We propose an extension of the BMS group, which we refer to as Weyl BMS or BMSW for short, that includes super-translations, local Weyl rescalings and arbitrary diffeomorphisms of the $2 \mathrm{~d}$ sphere metric. After generalizing the Barnich-Troessaert bracket, we show that the Noether charges of the BMSW group provide a centerless representation of the BMSW Lie algebra at every cross section of null infinity. This result is tantamount to proving that the flux-balance laws for the Noether charges imply the validity of the asymptotic Einstein's equations at null infinity. The extension requires a holographic renormalization procedure, which we construct without any dependence on background fields. The renormalized phase space of null infinity reveals new pairs of conjugate variables. Finally, we show that BMSW group elements label the gravitational vacua.

KeYwords: Models of Quantum Gravity, Space-Time Symmetries, Classical Theories of Gravity

ArXiv EPrinT: 2104.05793

In memory of Ezra Ted Newman, and the exploration of the heavens

that he so passionately pursued 


\section{Contents}

1 Introduction 1

2 Summary of the results 5

2.1 Flux-balance relation 6

2.2 Future null infinity 9

$\begin{array}{ll}2.3 \text { Phase space renormalization } & 11\end{array}$

$\begin{array}{lll}2.4 & \text { BMSW group and Einstein's equations } & 13\end{array}$

3 Geometric interpretation of the expansion coefficients 16

$\begin{array}{lll}4 & \text { The BMSW group } & 17\end{array}$

$\begin{array}{ll}4.1 \text { Residual diffeomorphisms } & 17\end{array}$

$\begin{array}{ll}4.2 \text { Boundary symmetry Lie algebra } & 19\end{array}$

4.3 Extended BMS and generalized BMS as Lie sub-algebroids 21

$\begin{array}{lll}\text { 4.3.1 Generalized bms } & 22\end{array}$

4.3.2 Extended bms 23

4.4 Action on the asymptotic phase space 25

$\begin{array}{ll}4.5 & \text { Covariant functionals }\end{array}$

4.6 On the definition of (angular) momentum 27

$\begin{array}{lll}4.7 & \text { Vacuum structure } & 28\end{array}$

5 Tetrad variables $\quad 29$

5.1 Charges and fluxes 30

5.2 Adapted tetrad 31

5.3 Residual gauge transformations 32

6 Noether charges: energy, Weyl and momentum 32

7 Noetherian fluxes: energy, Weyl and momentum 34

$\begin{array}{lll}7.1 & \text { Energy Noetherian flux } & 36\end{array}$

$\begin{array}{lll}7.2 & \text { Weyl Noetherian flux } & 36\end{array}$

$\begin{array}{lll}7.3 & \text { Momentum Noetherian flux } & 37\end{array}$

8 Einstein's equations from flux-balance laws $\quad 38$

8.1 Einstein's equations in Bondi gauge 38

$\begin{array}{lll}8.2 & \text { Flux-balance laws } & 39\end{array}$

8.2.1 Energy flux-balance $\quad 40$

8.2.2 Weyl flux-balance 41

8.2.3 Momentum flux-balance 43 
9 Charge and flux renormalization

$\begin{array}{lll}9.1 & \text { Renormalized charges } & 46\end{array}$

9.2 Relation to Barnich-Troessaert charges 46

9.3 Renormalized Noetherian fluxes 48

$\begin{array}{ll}10 \text { Conclusions } & 49\end{array}$

A Asymptotic expansions $\quad 51$

B Derivation the symplectic flux $\quad \mathbf{5 2}$

$\begin{array}{lll}\text { B.1 Asymptotic expansion of the temporal flux } & 52\end{array}$

B.2 Asymptotic expansion of the radial flux 54

$\begin{array}{ll}\text { C Variations of fields } & 55\end{array}$

$\begin{array}{ll}\text { D Weyl scalars in Bondi gauge } & 56\end{array}$

\section{Introduction}

Since the seminal work of Emmy Noether [1], revealing the deep connection between symmetries and charge conservation, the investigation of the symmetries in Nature has driven the most successful and dramatic breakthroughs in all fields of physics. Despite this long history, the study of symmetries does not cease to reveal surprising features of physical systems. One of the most notable examples is surely the central role of symmetries in gravitational systems.

The study of the gravitational group of symmetry at null infinity, the BMS group, dates back to the sixties [2-5] (see [6] for a review). However, about fifty years later, a surprising relationship between super-translation symmetry, the soft graviton theorem of Weinberg [7], and the displacement memory effect [8,9] was discovered [10] and elaborated in terms of a so-called infrared triangle [11] (see [12, 13] for reviews). Shortly after, the existence of a subleading soft graviton theorem was shown in [14] and it was related to the spin memory effect by [15]. This subleading soft graviton theorem was interpreted as a Ward identity for an extension of the original BMS group [16, 17], called the generalized BMS group, featuring super-Lorentz transformations. This group was studied canonically in [18], where it was shown that the vacua transitions generated by the generalized BMS group are related to the refraction memory effect. A proposal for a possible relation between vacua transition and spin memory effect was given in [19]. Overall, this seems to suggest a picture of an infrared square rather than a triangle [18]. More recently, a connection between this generalized symmetry group and another extension of BMS called extended BMS [20, 21] was revealed through the shadow transform [22]. The new framework of "celestial holography" has originated from these correspondences. It represents an approach 
aimed at defining the quantum gravity $S$-matrix by combining CFT techniques and the BMS symmetries of null infinity [12, 22-25].

Simultaneously, BMS-like symmetries have been investigated at finite distances for nonextreme black hole horizons [26-28], as well as more general null boundaries [29-32]. This renewed interest in understanding the symmetries of gravity requires developing a general framework that provides clear guidelines to relate the transformation properties of (quasilocal) physical observables to the dynamical content of the gravitational field equations.

With this in mind, the notion of corner symmetry algebra for local gravitational subsystems [33-35] has been proposed and provides an algebraic definition of gravitational subsystems. In fact, a new approach to quantum gravity has been advocated in [3643], where states of quantum geometry are built as representation states of the corner symmetry algebra.

Since corner symmetries label states of the system without reference to time, one has to wonder: how is the dynamics encoded? The proposal first sketched in [37] is to recast the quantum dynamics of the gravitational field in terms of flux-balance laws for the corresponding quantum charges. It then becomes crucial to understand how the corner symmetry algebra extended by super-translations relates to the flux-balance laws associated with the presence of (null) boundaries. The key idea developed here and formulated more generally in [44] is to identify the flux-balance laws with a canonical representation of the symmetry algebra. This relates the local-holographic description of the bulk gravitational dynamics and the boundary symmetry algebra. In the present paper we focus on developing these connections for physical observables on the celestial sphere, since asymptotic null infinity provides a perfect arena to test the viability and the potential of this new approach.

At the core of this program is the idea that each local gravitational sub-system can be understood as an open Hamiltonian system [45], where the passage of gravitational (and matter) radiation across the interface between subregions determines the evolution in time of the charges associated to the corner symmetry algebra. In such a case, there are two fundamental problems to address: first, one needs to choose a split of the Hamiltonian action of diffeomorphisms between charge and flux; second, we have to find a notion of bracket that defines the action of symmetry transformations and the time evolution dynamics of the phase space variables, like the Poisson bracket for a closed Hamiltonian system.

Our construction's capstone, developed in [44], is the introduction of the Noetherian split, which allows us to define charges and fluxes in an unambiguous way from a given Lagrangian. This split can be viewed as a formalization of the Wald-Zoupas prescription [46]. This construction builds upon the correspondence between boundary Lagrangian and corner symplectic potential [38], the concept of anomaly introduced in [29] and the important work [47], who derived the relationship between anomaly and cocycles. The Noetherian split is also instrumental for the derivation, based on first principles [44], of a new canonical bracket of the symmetry charges that generalizes the one introduced by Barnich and Troessaert in $[20,21,48]$. The new bracket resolves the ambiguity related to the handling of the non-integrable contributions and the presence of 2-cocycles [21, 47, 49-55]. In particular, we show following $[44,47]$ that the bracket provides a faithful and centerless representation of the algebra of vector fields generating the symmetry transformations. This is an essential 
and necessary step towards quantization: to have a well-defined quantization prescription, one needs to have a direct equivalence between path integral and canonical quantization, which means that the choice of Lagrangian should determine the charge algebra. And we also need to have a symmetry algebra with no field-dependent cocycles, i.e., we need a symmetry algebra not a symmetry algebroid. These semiclassical properties are achieved by our construction.

The flux-balance laws for mass and (angular) momentum aspects play a crucial role in general relativity's physical interpretation. These are usually derived using Einstein's equations (EEs) [56]. Covariant phase space methods [46, 57-61] then elevate these laws to relations among canonical generators of the asymptotic symmetries. Here we show that covariant phase space methods are much more powerful if adequately used: the flux-balance laws can be derived from the symmetries of the phase space structure and properties of the symplectic 2-form. In other words, working off-shell of the EEs, we show how the charge bracket defined on a general corner is related to Einstein's tensor's projection on the given 2-sphere.

The connection between symmetries and EEs means that the bigger the symmetry group the more equations of motion can be accessed. If one uses at null infinity the original BMS group, then only the evolution equations for the energy can be derived. To obtain the momentum evolution equations, it is required instead to use the generalized BMS group $[16,18,62]$. To have access to the rest of the asymptotic equations, one needs to further extend the asymptotic symmetry group to include conformal transformations of the metric on the 2-sphere. This leads us to the introduction of the Weyl BMS group or, shortly, the BMSW group, whose infinitesimal generators are shown to satisfy a Lie algebra, which corresponds to the maximally extended sub-algebra of the full diffeomorphism algebra [63] of null infinity $\mathcal{I}$ associated to a sphere embedded in $\mathcal{I}$. The BMSW group merges together the two different BMS extensions $[18,48]$ proposed in the literature, and we show explicitly how these previous extensions can be recovered from it. The larger BMSW group gives us access to more equations of motions. In particular, the renormalized charge bracket for every generator in the BMSW group allows us to recover up to eight of the ten asymptotic $\mathrm{EE}$, and not just the three flux-balance equations for energy and momentum at $\mathcal{I}$.

Moreover, the explicit inclusion of conformal transformations in the symmetry group of null infinity allows us to introduce a new Weyl charge, disentangling the super-rotation and super-boost components of the super-Lorentz transformations canonically studied in [18]. In this way, the momentum charge becomes a Hamiltonian charge with vanishing symplectic flux, providing a preferred notion of angular momentum (see e.g. [56, 64-68] for a recent debate on this ambiguity). An important reflection of this feature of the BMSW group is the fact that the orbits of Minkowski spacetime, under the finite action of the group generators, yield non-equivalent vacua of asymptotically flat spacetimes whose degeneracy is labelled also by the sphere diffeomorphisms, in addition to the super-translation and the conformal transformation fields as shown in [69].

In the language of $[70,71]$, this conformal extension means that the universal structure on $\mathcal{I}$ is reduced from a null vector and degenerate metric (original BMS) to a (thermal) Carroll structure (BMSW) [28, 31, 72, 73]; see also [74, 75] for an intrinsic and conformally invariant geometrical description of null infinity. 
As for previously considered extensions of the BMS group [16, 18, 20], the BMSW extension is also confronted with radially divergent charges at face value. While the derivation of the Einstein's equations is insensitive to these divergences, it is however an important consistency requirement that all charges associated with asymptotic symmetries should be finite. By exploiting the results of [38], which relate the introduction of a boundary Lagrangian to a shift of the symplectic potential by a corner term in an unambiguous manner, we perform a phase space renormalization for boundary conditions admitting the BMSW symmetry group. We explicitly show that all Noether charges and fluxes become finite. This way, the renormalization procedure extends the results of Compere et al. [18, 51] from the generalized BMS to the BMSW group and it reveals an extra pair of conjugate variables parametrizing the phase space of null infinity represented by the 2 -sphere scale factor and the Noetherian energy charge aspect. At the same time, quasi-local and asymptotic charges are now on same footing thanks to renormalization, and insights from the study of the phase space of null hypersurfaces [29, 76-81] can be imported to study extensions of the symplectic structure of $\mathcal{I}[62,82]$.

The outline of the paper is as follows. We start with a comprehensive summary of this paper and its companion paper [44] in section 2. This section is self-consistent, and gives a detailed account of the results obtained. Section 3 provides a geometric interpretation of the asymptotic expansion of the components of the Bondi metric. Section 4 contains the derivation $a b$ initio of the BMSW group and its relation with the original, extended and generalized BMS groups. In section 5, we introduce the Einstein-Cartan Lagrangian, which we use to compute the Noether charges and fluxes associated to the bmsw generators in section 6 and 7, respectively. Section 8 provides the derivation of the asymptotic EEs from the flux-balance relations, and section 9 shows the renormalization procedure for charges and fluxes. We also provide the construction of the boundary Lagrangian that yields the Noetherian construction of the Barnich-Troessaert charges. We conclude with a discussion and future endeavours in section 10. Four appendices collect technical results about asymptotic expansions in appendix A, the derivation of the flux formulae in appendix B, variation of the fields in appendix C, and about Weyl scalars in appendix D.

Notation \& nomenclature: we use units $8 \pi G=c=1$. We use Greek letters for spacetime indices, uppercase Latin letters $\{A, B, C, \ldots\}$ to label coordinates over the twodimensional sphere, uppercase Latin letters $\{I, J, \ldots\}$ for internal Lorentz indices, and lowercase $\{i, j, \ldots\}$ for the dyad on the sphere. The notation $T_{\langle A B\rangle}$ means the symmetric trace-free components of a given tensor $T$. Given a vector field $V=V^{\mu} \partial_{\mu}$, we use the notation $V[\cdot]=V^{\mu} \partial_{\mu}(\cdot)$. We denote $\dot{F}=\partial_{u} F$ and $F^{\prime}=\partial_{r} F$. Equations evaluated on-shell of the equations of motion are characterized by the symbol $\hat{=}$. The symbol $\stackrel{\mathcal{I}}{=}$ means that the right-hand side is evaluated at future null infinity $\mathcal{I}$. A bar over a quantity denotes its leading asymptotic value. We denote by $o\left(r^{x}\right)$ all the terms of order $n<x$ and by $\mathcal{O}\left(r^{x}\right)$ a term of order $n \leq x$, with $x, n \in \mathbb{Z}$.

We call the charge associated to the $\operatorname{Diff}(S)$ component of the boundary symmetry group "momentum charge" rather than "angular momentum charge", as customary in the previous literature. This different nomenclature reflects the (local) holographic perspective 
advocated in [37-39, 83], where the corner symmetry charge associated to diffeomorphisms tangent to the corner 2-sphere, being that at finite or infinite distance, has a clear interpretation as a momentum charge. Furthermore, from the point of view of fluid/gravity correspondence [84, 85], this charge has the interpretation of the fluid momenta.

\section{Summary of the results}

In this section, we describe some results of [44] which are needed in the following, and summarize the main results of this paper, namely

1. the extension of the BMS group including Weyl conformal rescaling;

2. the derivation of the asymptotic Einstein's equations at null infinity from flux-balance laws;

3. the definition of a procedure for phase space renormalization, yielding finite expressions for the canonical charges and fluxes;

4. the Noetherian derivation of Barnich-Troessaert BMS charges by a shifted Lagrangian;

5. the labelling of the vacua degeneracy spanned by the BMSW group by four arbitrary functions on the sphere, corresponding to super-translations, sphere diffeomorphisms and conformal transformations.

Let us start by introducing some main elements of the covariant phase space formalism. We denote by $d$ the spacetime differential and by $\delta$ the field space differential. As shown in [38], using the Anderson homotopy operators [86], it is possible to associate a unique symplectic potential $\theta=\theta_{L}$ to a given Lagrangian $L$. This potential is such that $\delta L=$ $\mathrm{d} \theta-E$, where $E$ labels the field equations, and it is used to define the pre-symplectic 2 -form $\omega=\delta \theta$. To be more precise, $\theta$ and $\omega$ are the integrands of the symplectic potentials and 2 -form, but with slight abuse of language we will refer to them in the same way. The actual pre-symplectic 2 -form is the integral on a 3 d hypersurface $\Sigma$,

$$
\Omega:=\int_{\Sigma} \delta \theta
$$

In this paper, we assume that $\Sigma$ is an hypersurface with boundary $S:=\partial \Sigma$, which we call the corner. We do not have to restrict $\Sigma$ to be space-like or null.

Given a vector field $\xi$ in spacetime, we denote $\iota_{\xi}$ the vector field contraction on spacetime forms and $I_{\xi}$ the field space contraction on field space forms. We also denote $£_{\xi}$ the Lie derivative and $\delta_{\xi}$ the corresponding field variation. Both Lie derivatives and field variations are related to the interior products by the Cartan's magic formula

$$
\mathcal{L}_{\xi}=\mathrm{d} \iota_{\xi}+\iota_{\xi} \mathrm{d}, \quad \delta_{\xi}=\delta I_{\xi}+I_{\xi} \delta
$$


For the gravitational field space, we have $\delta_{\xi} g_{\mu \nu}=\mathcal{L}_{\xi} g_{\mu \nu}$. In the following, we will be interested in field-dependent diffeomorphisms, ${ }^{1}$ hence $\delta \xi \neq 0$. In this case, it is necessary to consider also the operator $I_{\delta \xi}$ which denotes the field contraction along a form-valued vector. Field-dependent diffeomorphisms appear inevitably when working in a fixed gauge, as is customary done in the study of asymptotic symmetries. Indeed, suppose we impose a set of gauge-fixing conditions $F^{i}\left(g_{\mu \nu}\right)=0$. Obviously, a field-independent vector field will not preserve the gauge, but a subset of field-dependent diffeomorphisms such that $\delta_{\xi} F^{i}=D_{\xi}{ }^{i}(F)$, where $D_{\xi}$ is a linear differential operator, will. This is what happens, as we will discuss below, when we chose the (partial) Bondi gauge $g_{r r}=g_{r A}=0$.

A key ingredient in the construction of [44] is the anomaly operator $\Delta_{\xi}$, first introduced in [29] and also studied in [47]. As shown in more details in [44], anomalies necessarily appear when one introduces boundary Lagrangians that depend on the extrinsic geometry, such as the normal or its derivative. Given a form $\omega$ both in spacetime and field space, its anomaly is given by the difference between the field space action and the spacetime Lie derivative

$$
\Delta_{\xi} \omega, \quad \text { with } \quad \Delta_{\xi}:=\left(\delta_{\xi}-£_{\xi}-I_{\delta \xi}\right) .
$$

In this paper, we concentrate on the Einstein-Cartan formulation, which is a gravity formulation defined by a covariant Lagrangian, and thus we have

$$
\Delta_{\xi} L=0
$$

This covariance property is in general broken by the phase space renormalization procedure, where in general a non-covariant boundary Lagrangian needs to be introduced. We will come back to this important aspect shortly.

\subsection{Flux-balance relation}

On-shell of the field equations of motion we have $E \hat{=} 0$, which implies the vanishing of the constraints $C_{\xi}$ defined by

$$
\mathrm{d} C_{\xi}=I_{\xi} E
$$

explicitly

$$
C_{\xi}=\xi^{\mu} G_{\mu}{ }^{\nu} \epsilon_{\nu}, \quad \epsilon_{\mu}=\iota_{\partial_{\mu}} \epsilon,
$$

where $G_{\mu \nu}$ is the Einstein tensor and $\epsilon$ is the volume form. In situations where (2.4) is satisfied, we can define the Noether charge aspect

$$
\mathrm{d} q_{\xi} \hat{=} I_{\xi} \theta-\iota_{\xi} L
$$

whose integral is the Noether charge,

$$
Q_{\xi}:=\int_{S} q_{\xi}
$$

\footnotetext{
${ }^{1}$ See, e.g., $[52,53]$ for an application in lower dimensional gravity of a field-dependent redefinition of the generator parameters in order to make the charges integrable.
} 
We also introduce the Noetherian flux

$$
\mathcal{F}_{\xi}:=\int_{S}\left(\iota_{\xi} \theta+q_{\delta \xi}\right)
$$

so that the fundamental canonical relation ${ }^{2}$

$$
-I_{\xi} \Omega \hat{=} \delta Q_{\xi}-\mathcal{F}_{\xi}
$$

is satisfied.

The main result of [44] (which build upon the results of [47]) is to use the covariant phase space formalism to show how the choice of a Lagrangian defines uniquely a Noetherian split for charges (2.8) and fluxes (2.9) which allows one to define flux-balance laws in terms of a bracket available for open Hamiltonian systems, even in the presence of anomalies. While the general derivation in [44, 47] accounts for the presence of anomalies in both the Lagrangian and symplectic potential, we are initially interested in an application of this bracket in the case where these vanish, like in the Einstein-Hilbert or Einstein-Cartan formulations. It is important to realize though that, even in the simpler case where a covariant Lagrangian is available, the anomaly of the Noether charge does not vanish, and one has in general

$$
\Delta_{\xi} Q_{\chi}=Q_{\delta_{\chi} \xi}-Q_{\llbracket \xi, \chi \rrbracket},
$$

where the modified bracket $\llbracket \cdot, \cdot \rrbracket$ is defined by

$$
\llbracket \xi, \chi \rrbracket:=[\xi, \chi]_{\text {Lie }}+\delta_{\chi} \xi-\delta_{\xi} \chi .
$$

This modification of the Lie bracket is necessary in order to take into account the field dependence of the transformation generators, such that the commutator of two field space variations is again given by the a symmetry transformation

$$
\left[\delta_{\xi}, \delta_{\chi}\right]=-\delta_{\llbracket \xi, \chi \rrbracket} .
$$

The key relation (2.11) can be used to show that the bracket

$$
\left\{Q_{\xi}, Q_{\chi}\right\}_{L}:=\delta_{\xi} Q_{\chi}-I_{\chi} \mathcal{F}_{\xi}+\int_{S} \iota_{\xi} \iota_{\chi} L
$$

satisfies the off-shell flux-balance relation

$$
\left\{Q_{\xi}, Q_{\chi}\right\}_{L}+Q_{\llbracket \xi, \chi \rrbracket}=-\int_{S} \iota_{\xi} C_{\chi} .
$$

The bracket (2.14) is a generalization of the bracket first considered by Barnich and Troessaert $[20,21,48]$, as it contains an extra term depending on the Lagrangian. The expression (2.14) is valid for non-anomalous Lagrangians and can be generalised to include Lagrangian anomalies. The generalized Barnich-Troessaert bracket (2.14) has been explicitly shown in [44] to satisfy the Jacobi identity in full generality, including the case when anomalies are present. Moreover, it satisfies the following two essential properties.

\footnotetext{
${ }^{2}$ The contraction $\left.I_{\xi} \Omega=\delta_{\xi}\right\lrcorner \Omega$ is also denoted $\Omega\left(\delta_{\xi}, \delta\right)$ in the literature, and sometimes $-\not H_{\xi}$.
} 
First, it is independent of the choice of boundary Lagrangian and corner symplectic potential $(\ell, \vartheta)$. Namely, if we consider a change of Lagrangian $L^{\prime}=L+\mathrm{d} \ell$ and symplectic potential $\theta^{\prime}-\theta=\delta \ell-\mathrm{d} \vartheta$, the new Noether charge and flux are related to the old ones by the following shifts

$$
Q_{\xi}^{\prime}-Q_{\xi}=\int_{S}\left(\iota_{\xi} \ell-I_{\xi} \vartheta\right), \quad \mathcal{F}_{\xi}^{\prime}-\mathcal{F}_{\xi}=\int_{S}\left(\delta \iota_{\xi} \ell-\delta_{\xi} \vartheta\right)
$$

What is remarkable in these formulae is the fact that, even if the boundary Lagrangian and corner symplectic potential break the covariance property of the original pair $(L, \theta)$ - like, in general, it is the case for holographic phase space renormalization (see section 2.3) all the anomaly contributions finally drop out of the shift, as shown in [44]. The shifts of charge and flux (2.16) preserves the fundamental relation (2.10) and they leave the l.h.s. of (2.15) invariant, namely

$$
\left\{Q_{\xi}^{\prime}, Q_{\chi}^{\prime}\right\}_{L^{\prime}}+Q_{\llbracket \xi, \chi \rrbracket}^{\prime}=\left\{Q_{\xi}, Q_{\chi}\right\}+Q_{\llbracket \xi, \chi \rrbracket} .
$$

It is important to keep in mind though that the shifted bracket is now modified by the boundary Lagrangian anomaly; explicitly, the shifted bracket now reads

$$
\left\{Q_{\xi}^{\prime}, Q_{\chi}^{\prime}\right\}_{L^{\prime}}=\delta_{\xi} Q_{\chi}^{\prime}-I_{\chi} \mathcal{F}_{\xi}^{\prime}+K_{(\xi, \chi)}^{L^{\prime}}
$$

where

$$
K_{(\xi, \chi)}^{L^{\prime}}=\int_{S}\left(\iota_{\chi} \Delta_{\xi} \ell-\iota_{\xi} \Delta_{\chi} \ell\right)+\int_{S} \iota_{\xi} \iota_{\chi} L^{\prime} .
$$

This expression of the cocycle component of the bracket in terms of the boundary anomaly was first derived by Speranza and Chandrasekaran in [47] (see also [87] for the inclusion of anomalies in the study of covariant phase space with boundaries in gauge theories). This means that the flux-balance relation (2.15) is also preserved under change of boundary Lagrangian. This invariance property guarantees that it can be used also in the case where the expressions for charges and fluxes are formally divergent. In fact, as we will anticipate in the next section, the phase space renormalization procedure amounts to a shift by a boundary Lagrangian so that the shifted symplectic potential $\theta^{\prime}$, and the corresponding shifted charges and fluxes (2.16), become finite. In this way, one can use the bracket (2.14) at finite distance and see that all the potentially divergent terms in the flux-balance law cancel each other so that one can then take the limit to null infinity. This strategy is indeed available in the Einstein-Cartan formulation with zero cosmological constant and it will be worked out in section 8 .

The second main property of the bracket (2.14) is the fact that it provides a representation of the commutator (2.13) when on-shell of the constraints. In particular, the flux-balance relation (2.15), when applied to null infinity, explicitly connects the symmetry algebra on the celestial sphere to the asymptotic Einstein's equations at $\mathcal{I}$. In other words, Einstein's equations at null infinity can be expressed as the demand that the Noether charges corresponding to the BMSW transformations, defined in section 4, form a representation of the BMSW algebra under the bracket (2.14) on every cross section of $\mathcal{I}$. One of the results of this paper is indeed to explicitly show that demanding

$$
\left\{Q_{\xi}, Q_{\chi}\right\}=-Q_{\llbracket \xi, \chi \rrbracket}
$$


implies the Einstein equation projected along the pair $(\xi, \chi)$. Finally, the relation $(2.15)$ can equivalently be written as

$$
\delta_{\xi} Q_{\chi}+Q_{\llbracket \xi, \chi \rrbracket} \hat{=} I_{\chi} \mathcal{F}_{\xi}-\int_{S} \iota_{\xi} \iota_{\chi} L,
$$

which expresses how the charges evolve under the infinitesimal translation by $\xi$, making it manifest why it is a flux balance relation.

\subsection{Future null infinity}

In order to present our main results concerning the renormalized phase space at $\mathcal{I}$, it is convenient to introduce first the Bondi formalism for the parametrization of future null infinity and a comparison between the boundary conditions we will impose and the original BMS boundary conditions. We use Bondi-Sachs coordinates $x^{\mu}=\left(u, r, \sigma^{A}\right)$, where $u$ labels outgoing null and twist-free geodesic congruences, $r$ is a parameter along these geodesics, which measures the sphere's radius, and $\sigma^{A}$ are coordinates on the 2-sphere. The metric in these coordinates can be conveniently parametrized as follows $[2,3,88]^{3}$

$$
\mathrm{d} s^{2}=-2 e^{2 \beta} \mathrm{d} u(\mathrm{~d} r+F \mathrm{~d} u)+r^{2} q_{A B}\left(\mathrm{~d} \sigma^{A}-U^{A} \mathrm{~d} u\right)\left(\mathrm{d} \sigma^{B}-U^{B} \mathrm{~d} u\right) .
$$

The metric $q_{A B}$ and its inverse $q^{A B}$ can be used to raise and lower the indices of tensors on the 2 -sphere. We denote $D_{A}$ its Levi-Civita covariant derivative, and $R_{A B C D}=q_{A[C} q_{D] B} R$ its Riemann tensor and Ricci scalar.

The Bondi gauge conditions are

$$
g_{r r}=0, \quad g_{r A}=0, \quad \partial_{r} \sqrt{q}=0,
$$

where $q=\operatorname{det} q_{A B}$. This gauge-fixing allows one to study the limit to future null infinity taking $r \rightarrow \infty$. The original BMS boundary conditions, or fall-off conditions, are [88, 89]

$$
g_{u r}=-1+\mathcal{O}\left(r^{-2}\right), \quad g_{u A}=\mathcal{O}(1), \quad g_{u u}=-1+\mathcal{O}\left(r^{-1}\right), \quad q_{A B}=\stackrel{\circ}{q}_{A B}+\mathcal{O}\left(r^{-1}\right),
$$

where $\stackrel{\circ}{q}_{A B}$ is the metric of the round 2-sphere. We wish to consider more relaxed boundary conditions, with the goal of finding a larger extension of the BMS group. To that end, we keep the first two conditions in (2.24), but allow for an arbitrary leading order metric $\bar{q}_{A B}$ in the last. The asymptotic form of the Einstein's equations then requires to relax also the condition on $g_{u u}$ for consistency. Accordingly, our proposal is

$$
g_{u r}=-1+\mathcal{O}\left(r^{-2}\right), \quad g_{u A}=\mathcal{O}(1), \quad g_{u u}=\mathcal{O}(1), \quad q_{A B}=\mathcal{O}(1) .
$$

From these asymptotic conditions, the metric coefficients have the following fall-off behavior,

$$
\begin{aligned}
& F=\bar{F}-\frac{M}{r}+o\left(r^{-1}\right), \\
& \beta=\frac{\bar{\beta}}{r^{2}}+o\left(r^{-2}\right),
\end{aligned}
$$

\footnotetext{
${ }^{3}$ With $F=V /(2 r)$ in terms of Sachs' original parametrization.
} 


$$
\begin{aligned}
U^{A} & =\frac{\bar{U}^{A}}{r^{2}}-\frac{2}{3 r^{3}} \bar{q}^{A B}\left(\bar{P}_{B}+C_{B C} \bar{U}^{C}+\partial_{B} \bar{\beta}\right)+o\left(r^{-3}\right), \\
q_{A B} & =\bar{q}_{A B}+\frac{1}{r} C_{A B}+\frac{1}{r^{2}}\left(D_{A B}+\frac{1}{4} \bar{q}_{A B} C_{C D} C^{C D}\right)+\frac{1}{r^{3}} E_{A B}+o\left(r^{-3}\right) .
\end{aligned}
$$

All functions here depend a priori on the 2-sphere coordinates and on retarded time $u$. $M$ is the Bondi mass aspect, and the asymptotic expansion of $U^{A}$ is chosen to insure that the momentum aspect $\bar{P}_{A}$ is the BMS Noether charge associated with super-Lorentz transformations; more on this below. The $2 \mathrm{~d}$ tensors $\left(C_{A B}, D_{A B}, E_{A B}\right)$ are symmetric and traceless as a consequence of the determinant gauge condition. The tensor $C_{A B}$ is (twice) the asymptotic shear of the null geodesic congruence. The tensor $D_{A B}$ is known to vanish if we demand smooth fall off, namely absence of logarithm terms (see, e.g., [6]). We will follow this assumption in this paper, and thus set from now on $D_{A B}=0$. The tensor $E_{A B}$ appears as the dominant term in the expansion of one of the components of the Weyl tensor, see appendix D.

Notice that the asymptotic expansion of the metric coefficients listed above is consistent to inverse cubic order. To see this one assigns a notion of conformal weight which is such that $[r]=-1$ and $\left[\mathrm{d} s^{2}\right]=-2$. This implies that $\left[q_{A B}\right]=0,\left[U^{A}\right]=0$ and $\left[C_{A B}\right]=1$ while $[F]=2,[\beta]=1$. This means that to achieve a cubic order expansion we only need to expand $F$ to order $r^{-1}$ since $[M]=3$ and similarly we only need to expand $\beta$ to order $r^{-2}$.

Indices in the asymptotic expansion are raised and lowered using the leading order metric $\bar{q}_{A B}$. We denote $\bar{D}_{A}$ and $\bar{R}_{A B C D}$ the associated covariant derivative and Riemann tensor. With this expansion, the asymptotic Einstein's equations give the following relations for the leading order terms (see section (8) below for details)

$$
\begin{aligned}
\partial_{u} \bar{q}_{A B} & =0, & \mathrm{E}_{\bar{F}} & :=\bar{R}-4 \bar{F}=0, \\
\mathrm{E}_{\bar{\beta}} & :=\bar{\beta}+\frac{1}{32} C_{A B} C^{A B}=0, & \mathrm{E}_{\bar{U}^{A}} & :=\bar{U}^{A}+\frac{1}{2} \bar{D}_{B} C^{A B}=0 .
\end{aligned}
$$

The first equation can be understood as a boundary condition, rather than a dynamical equation. The remaining three equations restrict the number of constraint-free data that one has to assign, and have therefore a completely dynamical meaning. ${ }^{4}$ As shown in section 8 , the time-derivative of $(2.27 \mathrm{~b})$ can be understood as flux-balance laws, associated to conformal rescaling transformations. The rest of the asymptotic equations are the conservation equations $\mathrm{E}_{M}=0=\mathrm{E}_{\bar{P}_{A}}$ for the mass and momenta aspects, with

$$
\begin{aligned}
\mathrm{E}_{M}:= & \dot{M}-\frac{1}{4} \bar{D}_{A} \bar{D}_{B} N^{A B}-\frac{1}{2} \bar{\Delta} \bar{F}+\frac{1}{8} N_{A B} N^{A B}, \\
\mathrm{E}_{\bar{P}_{A}}:= & \dot{\bar{P}}_{A}-\bar{D}_{A} M-\frac{1}{8} \bar{D}_{A}\left(C^{B C} N_{C B}\right)-C_{A B} \partial^{B} \bar{F} \\
& -\frac{1}{4} \bar{D}_{C}\left(\bar{D}_{A} \bar{D}_{B} C^{B C}-\bar{D}^{C} \bar{D}^{B} C_{A B}\right) \\
& -\frac{1}{4} \bar{D}_{B}\left(N^{B C} C_{A C}-C^{B C} N_{A C}\right)+\frac{1}{4} N^{B C} \bar{D}_{A} C_{B C} .
\end{aligned}
$$

\footnotetext{
${ }^{4}$ We also notice that the first two imply that the leading order of $g_{u u}$ and $q_{A B}$ is $u$-independent.
} 
These can also be understood as flux-balance laws associated to super-translation and sphere diffeomorphism, respectively.

There are two other declinations of the BMS boundary conditions in the literature. In $[20,48,90]$, the authors allow for a divergent term in $g_{u u}$ of order $r$, while restricting the leading order metric to be conformally related to the round metric $\stackrel{\circ}{A B}_{A B}$. In this setup, the asymptotic Einstein's equations allow for a $u$-dependence in the conformal factor. We will refer to these boundary conditions as the extended BMS boundary conditions and the corresponding symmetry group as the extended BMS group. Another set of boundary conditions proposed in $[16,18]$ is similar to the one described here. Except that it fixes the determinant of the boundary metric $\sqrt{\bar{q}}$ while allowing for an arbitrary variation of its conformal class $\sqrt{\bar{q}} \bar{q}^{A B}$. We will refer to these boundary conditions as the generalized BMS boundary condition and the symmetry group as the generalized BMS group.

Our proposal is more general than $[16,18]$, in that we allow also the determinant of the leading metric to vary. This introduces additional divergences in the symplectic potential, but we will show that it is possible to renormalize them as well, thus extending the results of [18] in the case of the generalized BMS group. However, it does not go as far as introducing a linear divergence in $g_{u u}$ and thus a time dependence of the leading order metric. One reason for this is that $g_{u u}=\mathcal{O}(1)$ appears to be sufficient to describe the physics of compact binaries [91, 92]. ${ }^{5}$ The consequences of these different boundary conditions on the asymptotic symmetry group are discussed in section 4.

\subsection{Phase space renormalization}

If a Lagrangian has a symplectic potential that diverges in an asymptotic limit, it can be tempting to strengthen the fall-off conditions in order to remove such divergences. However, doing so arbitrarily restricts the phase space definition and can set to zero quantities of physical interest. One may ask whether a more general procedure of renormalization exists, that allows one to identify from first principles what are the weakest fall-off conditions acceptable. This is the idea behind the holographic renormalization procedure.

Holographic renormalization techniques for conserved charges have been developed in the context of AdS/CFT in [51,93-95]. These techniques have been revisited in the context of flat space asymptotic by [96] for QED in all dimensions and most notably by [18] for the case of $4 \mathrm{~d}$ gravity. These methods rely on adding to the gravitational action a set of covariant boundary counter-terms, and have been applied to the case of $\Lambda$-BMS in 4 dimensions in [51]. The main new ingredient is the focus on the renormalization of the symplectic potential and not simply on the renormalization of the Lagrangian. Here we follow an approach closer to [18], where the phase space renormalization is defined by the addition of a boundary Lagrangian and related corner symplectic potential [38] such that the shifted pair $\left(L^{\prime}, \theta^{\prime}\right)$ is finite. Our approach relies on the choice of a particular foliation of the boundary induced by the choice of Bondi coordinates, but it does not use background fields.

\footnotetext{
${ }^{5}$ The Einstein's equations can be perturbatively solved in harmonic gauge under the assumption of no incoming radiation [91]. Transforming to Bondi or Newman-Unti coordinates one obtains $g_{u u}=\mathcal{O}(1)[92]$.
} 
Let us summarize the results of the procedure worked out in detail in section 9. Suppose that one starts from a covariant Lagrangian $L$ with covariant symplectic potential $\theta$ that possesses some divergences. The goal is to define a finite renormalized Lagrangian $L_{R}$ with symplectic potential $\theta_{R}$ by the subtraction of a boundary divergent Lagrangian $\ell_{\text {div }}$ with corner symplectic potential term $\vartheta_{\text {div }}$. The subtraction terms may have nonvanishing anomalies $\Delta_{\xi} \ell_{\text {div }}$ and $\Delta_{\xi} \vartheta_{\text {div }}$ - let us stress again that the source of these possible anomalies is the field dependence of the transformation parameters and eventually the coordinates dependence, and not due to the introduction of background structures; moreover, the anomalies do not enter the expressions (2.16) for the shifted charges and fluxes. The renormalized quantities are then

$$
L_{R}:=L+\mathrm{d} \ell_{\mathrm{div}}, \quad \theta_{R}:=\theta-\mathrm{d} \vartheta_{\mathrm{div}}+\delta \ell_{\mathrm{div}} .
$$

We can thus define, from the general expressions in (2.16), the renormalized Noether charge

$$
Q_{\xi}^{R}=Q_{\xi}+\int_{S}\left(\iota_{\xi} \ell_{\mathrm{div}}-I_{\xi} \vartheta_{\mathrm{div}}\right)
$$

and the renormalized Noetherian flux

$$
\mathcal{F}_{\xi}^{R}=\mathcal{F}_{\xi}+\int_{S}\left(\delta \iota_{\xi} \ell_{\mathrm{div}}-\delta_{\xi} \vartheta_{\mathrm{div}}\right)
$$

In section 9 , we show that the divergent term of the symplectic potential at $\mathcal{I}$ can be written as

$$
\theta_{\text {div }}=\mathrm{d} \vartheta_{\text {div }}
$$

with $\ell_{\text {div }}=0$ on-shell of $\partial_{u} \bar{q}_{A B}=0$ and

$$
\vartheta_{\text {div }}=\left(\frac{r^{2}}{2} \delta \sqrt{\bar{q}}-\frac{r}{4} \sqrt{\bar{q}} C^{A B} \delta \bar{q}_{A B}\right) \mathrm{d}^{2} \sigma+r \bar{\vartheta} \wedge \mathrm{d} u,
$$

where $\bar{\vartheta}$ is a one form on $S$ such that

$$
\mathrm{d} \bar{\vartheta}=\frac{1}{2} \delta(\sqrt{\bar{q}} \bar{R}) .
$$

Its explicit expression is given in (9.5).

Furthermore, our phase space renormalization procedure yields a finite expression for the symplectic 2 -form at null infinity ${ }^{6}$

$$
\begin{aligned}
& \Omega_{\mathcal{I}}^{R}=-\int_{\mathcal{I}} \delta \theta_{R}^{r} \epsilon_{r}=\int_{\mathcal{I}} \frac{1}{4} \delta N_{A B} \curlywedge \delta\left(\sqrt{\bar{q}} C^{A B}\right)-\frac{1}{2} \delta\left(\bar{F} C_{A B}+D_{\langle A} \bar{U}_{B\rangle}\right) \curlywedge \delta\left(\sqrt{\bar{q}} \bar{q}^{A B}\right) \\
&\left.+\delta\left(M-\frac{1}{2} D_{A} \bar{U}^{A}\right) \curlywedge \delta \sqrt{\bar{q}}\right] \mathrm{d} u \mathrm{~d}^{2} \sigma,
\end{aligned}
$$

where we use the curly wedge symbol to denote a wedge in field space. If we restrict to the original BMS boundary conditions, only the first pair of conjugate variables in (2.36)

\footnotetext{
${ }^{6}$ In the Bondi coordinates we will use below, we pick an orientation with $\epsilon=e^{2 \beta} r^{2} \sqrt{q} \mathrm{~d} u \mathrm{~d} r \mathrm{~d}^{2} \sigma$, which means that $\epsilon_{u}=e^{2 \beta} r^{2} \sqrt{q} \mathrm{~d} r \mathrm{~d}^{2} \sigma$ while $\epsilon_{r}=-e^{2 \beta} r^{2} \sqrt{q} \mathrm{~d} u \mathrm{~d}^{2} \sigma$ contains a minus sign.
} 
remains and we recover the original radiative phase space of $[71,97]$. The BMSW extension proposed here allows us to reveal two extra pairs so that the phase space at $\mathcal{I}$ on which the BMSW group acts is parametrized by the set of conjugate variables

$$
N_{A B} \leftrightarrow \sqrt{\bar{q}} C^{A B}, \quad\left(\bar{F} C_{A B}+D_{\langle A} \bar{U}_{B\rangle}\right) \leftrightarrow \sqrt{\bar{q}} \bar{q}^{A B}, \quad\left(M-\frac{1}{2} D_{A} \bar{U}^{A}\right) \leftrightarrow \sqrt{\bar{q}} .
$$

Note that, in each pair, one of the variable is always a density. The second pair, which contains the complex structure on the sphere, as $\sqrt{\bar{q}} \bar{q}^{A B}$ is conformally invariant, ${ }^{7}$ was already revealed in [18]. The symplectic potential of [18] can be obtained up to a corner term ${ }^{8}$ from ours by imposing $\delta \sqrt{\bar{q}}=0$. The third pair contains the sphere conformal scale $\sqrt{\bar{q}}$, which is conjugate to the renormalized Noetherian energy charge aspect (see (2.41) below).

\subsection{BMSW group and Einstein's equations}

The BMSW group, which is studied in more details in section 4, is given by the semi-direct product $\left(\operatorname{Diff}(S) \ltimes \mathbb{R}_{W}^{S}\right) \ltimes \mathbb{R}_{T}^{S}$ and it is generated by the vector fields $\left(\xi_{T}, \xi_{W}, \xi_{Y}\right)$ given by (see (6.2) for the complete expression)

$$
\begin{aligned}
\xi_{T} & :=T \partial_{u}+o(1) \\
\xi_{W} & :=W\left(u \partial_{u}-r \partial_{r}\right)+o(1), \\
\xi_{Y} & :=Y^{A} \partial_{A} .
\end{aligned}
$$

where the $o(1)$ refers to terms that vanish when $r \rightarrow \infty$. $T$ and $W$ are time independent functions on $S$, while $Y=Y^{A} \partial_{A}$ is a time independent vector field on $S$. We will refer to $\xi_{T}$ as the super-translation, $\xi_{W}$ as the Weyl super-boost, and $\xi_{Y}$ as the $\operatorname{Diff}(S)$ vector fields. A general BMSW vector field is then given by $\xi_{(T, W, Y)}:=\xi_{T}+\xi_{W}+\xi_{Y}$.

The bmsw Lie algebra structure is defined by

$$
\llbracket \xi_{\left(T_{1}, W_{1}, Y_{1}\right)}, \xi_{\left(T_{2}, W_{2}, Y_{2}\right)} \rrbracket=\xi_{\left(T_{12}, W_{12}, Y_{12}\right)},
$$

where

$$
\begin{aligned}
Y_{12} & =\left[Y_{1}, Y_{2}\right]_{\text {Lie }}, \\
W_{12} & =Y_{1}\left[W_{2}\right]-Y_{2}\left[W_{1}\right], \\
T_{12} & =Y_{1}\left[T_{2}\right]-W_{1} T_{2}-\left(Y_{2}\left[T_{1}\right]-W_{2} T_{1}\right),
\end{aligned}
$$

and $[\cdot, \cdot]_{\text {Lie }}$ denotes the sphere Lie bracket.

The renormalization procedure, complemented by the use of the asymptotic Einstein's equations for $\bar{F}$ in (2.27a) and for $\bar{U}^{A}$ in (2.27b) (necessary to renormalize $Q_{Y}$ ), yields the following finite expressions for the energy (or super-translations), Weyl and momentum (or $\operatorname{diff}(S))$ Noether charges:

$$
Q_{T}^{R}=\int_{S} \sqrt{\bar{q}} T\left(M-\frac{1}{2} \bar{D}_{A} \bar{U}^{A}\right)
$$

\footnotetext{
${ }^{7}$ If one denote $\epsilon_{A B}$ the Levi-Civita symbol, the sphere complex structure is $J_{A}^{B}:=\sqrt{\bar{q}} \epsilon_{A C} \bar{q}^{C B}$, and we can check that it satisfies $J^{2}=-1$ and $D_{A} J_{B}{ }^{C}=0$.

${ }^{8}$ The relationship between the generalized bms symplectic structure of Compere et al. [18] and ours is $\Omega_{\mathrm{bmsw}}=\Omega_{\mathrm{bms}}-\frac{1}{4} \int_{\partial \mathcal{I}} \delta C^{A B} \curlywedge \delta C_{A B}$ after imposing $\delta \sqrt{\bar{q}}=0$.
} 


$$
\begin{aligned}
Q_{W}^{R} & =\int_{S} \sqrt{\bar{q}} W\left[4 \bar{\beta}+u\left(M-\frac{1}{2} \bar{D}_{A} \bar{U}^{A}\right)\right], \\
Q_{Y}^{R} & =\int_{S} \sqrt{\bar{q}} Y^{A}\left(\bar{P}_{A}+2 \bar{D}_{A} \bar{\beta}\right)
\end{aligned}
$$

where $\left(T, W, Y^{A}\right)$ are arbitrary functions on the 2-sphere. The full renormalized Noether charge then reads

$$
Q_{(T, W, Y)}^{R}=\int_{S} \sqrt{\bar{q}}\left[(T+u W)\left(M-\frac{1}{2} \bar{D}_{A} \bar{U}^{A}\right)+\left(2 W-\bar{D}_{A} Y^{A}\right) 2 \bar{\beta}+Y^{A} \bar{P}_{A}\right] .
$$

The charges (2.41), (2.42), (2.43), together with the corresponding finite expressions for the Noether fluxes $\mathcal{F}_{T}^{R}, \mathcal{F}_{W}^{R}, \mathcal{F}_{Y}^{R}$, given in section 9, can be used to have a well defined notion of charge bracket at null infinity so that the validity of the asymptotic Einstein's equations is equivalent to having a centerless representation of the residual diffeomorphism algebra at each cross-section of $\mathcal{I}$, namely

$$
\left\{Q_{\xi}^{R}, Q_{\chi}^{R}\right\}=-Q_{\llbracket \xi, \chi \rrbracket}^{R} .
$$

This result extends the one of [51], where the generalized BMS surface charge algebra of $[16,18]$ was shown to close only at the corners $u \rightarrow \pm \infty$ of $\mathcal{I}$. In particular, it shows that the BMSW charge algebra is realized as the symmetry algebra of asymptotically flat spacetimes at all times.

One important subtlety is that the bmsw charge associated to the sphere diffeomorphisms $\operatorname{diff}(S)$ is not the same as the bms super-Lorentz charge appearing in [18]. For instance, the Noetherian flux $\mathcal{F}_{Y}^{R}$ for bmsw $\operatorname{diff}(S)$ vanishes, while the bms flux $\mathcal{F}_{Y}^{R \text {-bms }}$ does not. This can be seen explicitly from the fact that a bms vector field can be written as

$$
\xi_{T}^{\mathrm{bms}}=\xi_{T}, \quad \xi_{Y}^{\mathrm{bms}}=\xi_{Y}+\xi_{W=\frac{1}{2} \bar{D}_{A} Y^{A}}
$$

By linearity this means that a bms super-Lorentz transformation is the sum of a bmsw sphere diffeomorphism transformation plus a Weyl super-boost. In particular, it implies that the bms super-translation and super-Lorentz charges are

$$
Q_{T}^{R-\mathrm{bms}}=Q_{T}^{R}, \quad Q_{Y}^{R-\mathrm{bms}}=Q_{Y}^{R}+Q_{W=\frac{1}{2} \bar{D}_{A} Y^{A}}^{R}
$$

Given this relation, it follows that the BMS charges also provide a centerless representation of the canonical algebra as in (2.45).

This conclusion seems to be in contrast with the statements of $[17,18,21]$ that the bms charges constructed by Barnich-Troessaert possess a non-trivial field-dependent 2-cocycle. It is also in tension with the statement of [51] that a centerless representation exists only at the corners of $\mathcal{I}$. The Barnich-Troessaert charges are given by

$$
Q_{(T, Y)}^{\mathrm{BT}}=\int_{S} \sqrt{\bar{q}}\left(2 \tau M+Y^{A} \bar{P}_{A}\right)
$$

where $\tau=T+\frac{u}{2} \bar{D}_{A} Y^{A}$. They were first derived in [21] using the Barnich-Brandt formalism [98] and later in [17] from a split following the prescription laid out by Wald-Zoupas [46]. 
To resolve the tension, we show that these charges are in fact Noether charges, obtained through the prescription (2.16) with boundary Lagrangian

$$
\ell=\sqrt{\bar{q}}\left(M-\frac{1}{8} C^{A B} N_{A B}\right) \mathrm{d} u \wedge \mathrm{d}^{2} \sigma .
$$

Furthermore, we prove that the non-central 2-cocycle appearing in [51] when computing the Barnich-Troessaert bracket of the charge (2.48) is nothing but the extra contribution (2.19) in the bracket (2.18) due to the non-covariance of the boundary term (2.49).

Therefore, we can understand the Barnich-Troessaert split between integrable and nonintegrable terms [21] as a Noetherian split for the boundary Lagrangian (2.49), and the appearance of a 2-cocycle in the charge bracket at a general cross section of $\mathcal{I}$ is simply a reflection of the fact that the original Barnich-Troessaert bracket does not take into account the contribution (2.19), which originates from the fact that the boundary Lagrangian (2.49) has an anomaly. In other words, if we wish to work with the Barnich-Troessaert charge bracket, the only way to avoid the appearance of a 2-cocycle is to apply the Noetherian split with a covariant boundary Lagrangian.

In the case of the Einstein-Cartan (or Einstein-Hilbert) Lagrangian, the only charge yielding a centerless representation of the bmsw Lie algebra is the Noether charge (2.44). The analog charge for the generalized BMS case is the Noether charge given by (9.13).

The option we propose to resolve the appearance of cocycles when working with a non-covariant Lagrangian is instead to include the anomalies in the definition of the charge bracket as in (2.18), in order to insure that the bracket does not involve field-dependent cocycles. This proposal allows us to introduce anomalies in a consistent manner both at the level of the charges and the bracket.

Finally, the vacuum sector can be constructed from the orbits of the BMSW group by acting with the group elements

$$
g_{(T, W, Y)}=e^{\delta_{T}} e^{\delta_{W}} e^{\delta_{Y}}
$$

on the phase space at null infinity. The infinitesimal transformations $\delta_{T}, \delta_{W}, \delta_{Y}$ are the ones generated respectively by the vector fields (2.38) and given explicitly in appendix C. The vacua $|T, W, Y\rangle=\hat{g}_{(T, W, Y)}|0\rangle$ are thus labelled by the two sphere diffeomorphisms arbitrary functions $Y^{A}$, in addition to the super-translations $T$, and conformal transformations $W$. We expect each factor entering the vacua definition to be related to a memory effect: the super-translation $T$ accounts for the displacement memory effect [8, 9]; the diffeomorphism accounts for a change of asymptotic conformal structure which could lead to a memory effect generalizing the spin memory effect [15]. We expect the Weyl rescaling factor $W$ to be related to the refraction memory effect [18], as this determines the vacuum value of the News tensor (see section 4.7). These open questions will be investigated elsewhere.

We conclude our summary with table 1, which shows the interplay among geometric data (the group generators), phase-space data (charges, fluxes and bracket), and dynamics (the Einstein's tensor). The table summarizes the remarkable property that the renormalized charge bracket (2.45) is equivalent to the asymptotic EEs by showing the detailed relation among the pair of bmsw generators, its associated renormalized charge bracket, and what components of the Einstein's tensor display the corresponding flux-balance laws. 


\begin{tabular}{|c|c|c|}
\hline BMSW generators $(\xi, \chi)$ & $\left\{Q_{\xi}^{R}, Q_{\chi}^{R}\right\}+Q_{\llbracket \xi, \chi \rrbracket}^{R}=0$ & Einstein's equations \\
\hline$\left(\partial_{u}, \xi_{T}\right)$ & $2 \mathrm{E}_{M}-\frac{1}{4} \bar{\Delta} \mathrm{E}_{\bar{F}}=0$ & $\xi_{T}^{\mu} G_{\mu}{ }^{r}=0$ \\
\hline$\left(\xi_{T}, \partial_{u}\right)$ & $2 \mathrm{E}_{M}+\bar{D}^{A} \dot{\mathrm{E}}_{\bar{U}_{A}}+\frac{1}{4} \bar{\Delta} \mathrm{E}_{\bar{F}}=0$ & $\xi_{T}^{u} G_{u}{ }^{r}-\xi_{T}^{r} G_{u}{ }^{u}=0$ \\
\hline$\left(\partial_{u}, \xi_{W}\right)$ & $\bar{D}^{A} \mathrm{E}_{\bar{U}_{A}}+u\left(2 \mathrm{E}_{M}-\frac{1}{4} \Delta \mathrm{E}_{\bar{F}}\right)=0$ & $\xi_{W}^{\mu} G_{\mu}{ }^{r}=0$ \\
\hline$\left(\xi_{W}, \partial_{u}\right)$ & $-\bar{D}^{A} \mathrm{E}_{\bar{U}_{A}}+u\left(2 \mathrm{E}_{M}+\bar{D}^{A} \dot{\mathrm{E}}_{\bar{U}_{A}}+\frac{1}{4} \Delta \mathrm{E}_{\bar{F}}\right)=0$ & $\xi_{W}^{u} G_{u}{ }^{r}-\xi_{W}^{r} G_{u}{ }^{u}=0$ \\
\hline$\left(\partial_{u}, \xi_{Y}\right)$ & $\mathrm{E}_{\bar{P}_{A}}+2 \bar{D}_{A} \dot{\mathrm{E}}_{\bar{\beta}}-2 \bar{F} \mathrm{E}_{\bar{U}_{A}}-\frac{1}{2} \bar{U}_{A} \mathrm{E}_{\bar{F}}=0$ & $\xi_{Y}^{\mu} G_{\mu}{ }^{r}=0$ \\
\hline$\left(\xi_{Y}, \partial_{u}\right)$ & $0=0$ & $0=0$ \\
\hline
\end{tabular}

Table 1. Synoptic overview of the flux-balance laws associated to the pair $(\xi, \chi)$ and their relation to Einstein's equations. We recall that by the property (2.17) the charge algebra is left unchanged by the renormalization procedure.

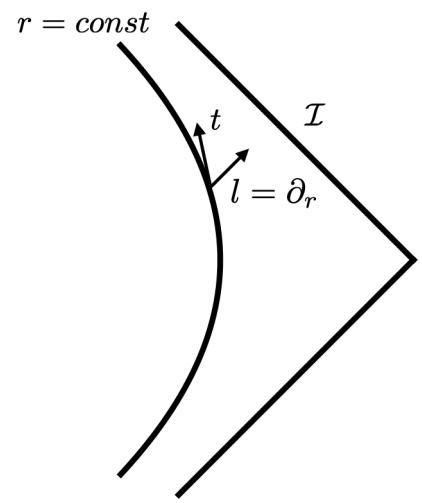

Figure 1. The figure shows an idealized $r=$ const hypersurface, the null vector $l$, which can be used to study its radial evolution, and the time-like vector $t$ tangent to the hypersurface.

\section{Geometric interpretation of the expansion coefficients}

Thanks to the coordinate gauge-fixing, all functions appearing in (2.26) acquire a precise geometric meaning, which is useful to recall here. This will allow us to further motivate our choice of momentum aspect $\bar{P}_{A}$, which unlike the other quantities, is not universal in the literature. To that end, let us denote

$$
l:=\partial_{r}, \quad l^{2}=0, \quad t:=\partial_{u}+U^{A} \partial_{A}, \quad t^{2}=-2 e^{2 \beta} F, \quad l_{\mu} t^{\mu}=-e^{2 \beta} .
$$

$l$ is the null vector tangent to the null geodesics which cut scri along the constant $u$ spheres. $t$ is the unique time-like vector which is transverse to $l$ and tangent to the constant $r$ hypersurfaces (see figure 1). Note that, upon compactification, it becomes a null generator at $\mathcal{I}$.

The first relevant geometric quantity is the extrinsic tensor of the null hypersurfaces normal to $l$. It is given by

$$
S_{A B}:=\frac{1}{2 r} \mathcal{L}_{l} g_{A B}=\frac{1}{2}\left(2+r \partial_{r}\right) q_{A B}=\bar{q}_{A B}+\frac{C_{A B}}{2 r}+o\left(r^{-2}\right) .
$$

From this expression we see that the null geodetic congruences have asymptotic shear $C_{A B}$ and asymptotic expansion 2, which is just the reflection of $r$ being the area radius. The 
second geometric quantity is the extrinsic curvature of the sphere $S$ within the time-like hypersurface of constant $r$. It is given by

$$
\begin{aligned}
K_{A B} & :=\frac{1}{2 r} \mathcal{L}_{t} g_{A B}=r\left(\frac{1}{2} \partial_{u} q_{A B}+D_{(A} U_{B)}\right) \\
& =\frac{1}{2} N_{A B}+\frac{1}{r}\left(\bar{D}_{(A} \bar{U}_{B)}+\frac{\bar{q}_{A B}}{8} \partial_{u} C^{2}\right)+o\left(r^{-1}\right),
\end{aligned}
$$

where $N_{A B}:=\partial_{u} C_{A B}$ is the Bondi news tensor and $C^{2}:=C_{A B} C^{A B}$. From this expression we see that the news tensor is the asymptotic extrinsic curvature. As a side remark, we note that the trace $q^{A B} K_{A B}=\mathcal{O}\left(r^{-1}\right)$ is sub-dominant.

The third quantity is the angular momentum aspect $\bar{P}_{A}$, which we refer to as momentum aspect as explained in the introduction, and which enters the expansion (2.26c). The relevant geometric quantity is the parallel transport of $t$ along $l$,

$$
\eta_{A}:=r e^{-2 \beta} \nabla_{l} t_{A}=r\left(\partial_{A} \beta+\frac{r^{2}}{2} e^{-2 \beta} q_{A B} \partial_{r} U^{B}\right)=-\bar{U}_{A}+\frac{1}{r}\left(\bar{P}_{A}+2 \partial_{A} \bar{\beta}\right)+o\left(r^{-1}\right) .
$$

This quantity is precisely the spin- 1 momentum of the geometric decomposition of the symplectic potential $[29,78,81] .^{9}$

Finally, from the point of view of the gravity-fluid duality [99], it is also useful to consider the velocity

$$
V^{A}=r^{2} e^{-2 \beta} U^{A}=\bar{U}^{A}-\frac{2}{3} \frac{\bar{q}^{A B}}{r}\left(\bar{P}_{B}+C_{B C} \bar{U}^{C}+\partial_{B} \bar{\beta}\right)+o\left(r^{-1}\right) .
$$

The reader will notice that the four quantities above have been suitably rescaled by appropriate factors of $r$, with respect to their natural geometric definitions. This was done so that their leading orders were finite.

\section{The BMSW group}

In this section we define the BMSW group, its Lie algebra structure, its boundary and bulk realizations and its relationship with the original BMS group, the generalized BMS group and the extended BMS groupoid.

\subsection{Residual diffeomorphisms}

The residual diffeomorphisms which define the boundary symmetry generators are defined by two sets of requirements, which are to be analyzed separately. These are:

(i) The preservation of the Bondi gauge-fixing (2.23):

$$
£_{\xi} g_{r r}=0, \quad £_{\xi} g_{r A}=0, \quad \partial_{r}\left(g^{A B} £_{\xi} g_{A B}\right)=0 .
$$

\footnotetext{
${ }^{9}$ If we introduce a second null vector $n$ transverse to $l$ and such that the space-like spaces orthogonal to the pair $(l, n)$ are tangent to the sphere, we have $\eta_{A}:=-r n^{\mu} \nabla_{A} l_{\mu}$. The quantity $n^{\mu} \nabla_{A} l_{\mu}$ contains the two transverse components of the spin- 1 momentum, and it is also related to the non-integrability of the $(l, n)$ planes and to the rotational 1-form of the isolated horizon framework; see discussion in [29, 81].
} 
These conditions are solved respectively restricting the components of $\xi$ to be of the form

$$
\xi^{u}=\tau, \quad \xi^{A}=Y^{A}-I^{A B} \partial_{B} \tau, \quad \xi^{r}=-r W+\frac{r}{2}\left(D_{A}\left(I^{A B} \partial_{B} \tau\right)+U^{A} \partial_{A} \tau\right),
$$

where $D_{A}$ is the covariant derivative associated with $q_{A B}$ and

$$
I^{A B}:=\int_{r}^{\infty} \frac{\mathrm{d} r^{\prime}}{r^{\prime 2}} e^{2 \beta} q^{A B}
$$

The parameters $\tau, Y^{A}$ and $W$ are, at this stage, arbitrary functions of $\left(u, \sigma^{A}\right)$ but not of $r$, and the Weyl factor $W$ is such that

$$
\delta_{\xi} \sqrt{q}=\left(D_{A} Y^{A}-2 W\right) \sqrt{q},
$$

where $D_{A} Y^{A}$ measures the variation of the sphere area element under a diffeomorphism, while $W$ labels a Weyl rescaling. This transformation rule, which distinguishes the rescaling due to tangent diffeomorphisms from the rescaling due to Weyl transformation, justifies with hindsight the parametrization (4.2) of $\xi^{r}$, which is a slight generalization of the procedure [3] (see [18, 20]). It is important to note that $D_{A} Y^{A}=\bar{D}_{A} Y^{A}$ is independent of $r$ due to the determinant condition in the Bondi gauge. This means that $\partial_{r}\left(\delta_{\xi} \sqrt{q}\right)=0$ which is equivalent to the last condition of (4.1). ${ }^{10}$

(ii) The preservation of the boundary conditions (2.25):

$$
\begin{array}{ll}
£_{\xi} g_{u r}=\mathcal{O}\left(r^{-2}\right), & £_{\xi} g_{u A}=\mathcal{O}(1) \\
£_{\xi} g_{u u}=\mathcal{O}(1), & £_{\xi} g_{A B}=\mathcal{O}\left(r^{2}\right) .
\end{array}
$$

These impose

$$
\partial_{u} Y^{A}=0, \quad \partial_{u} W=0,
$$

and restrict $\tau$ to be a linear function of $u$ given by

$$
\tau=T+u W, \quad \partial_{u} T=0, \quad \dot{\tau}=W, \quad \ddot{\tau}=0 .
$$

The BMSW vector fields preserving $(i)-(i i)$ depend on two arbitrary functions $(T, W)$ on the 2-sphere, equivalently on a linear function of time $\tau=T+u W$, and on a vector $Y=Y^{A} \partial_{A}$ on $S$. They will be denoted $\xi_{(\tau, Y)}$ or $\xi_{(T, W, Y)}$. The gauge parameter $T\left(\sigma^{A}\right)$ is associated to the asymptotic super-translations, $Y^{A}\left(\sigma^{A}\right)$ represent asymptotic diffeomorphisms of the celestial sphere $S$, while $W\left(\sigma^{A}\right)$ corresponds to Weyl rescaling of the celestial sphere.

${ }^{10}$ This follows from

$$
\delta_{\xi} \ln \sqrt{q}=\frac{1}{2} g^{A B} \delta_{\xi} g_{A B}=\frac{1}{2} g^{A B} \mathcal{L}_{\xi} g_{A B}=2 \frac{\xi^{r}}{r}+D_{A} \xi^{A}-U^{A} \partial_{A} \xi^{u} .
$$


All the asymptotic symmetry groups studied so far in flat space holography (see [100] for an overview), such as the original BMS group [5, 88, 89], the extended BMS group introduced by Barnich-Troessaert [48, 90,101] and the generalized BMS group put forward by Campiglia and Laddha [16] and studied canonically by Compere et al. [18], appear as a restriction of the BMSW group. For instance, the generalized BMS group $\operatorname{Diff}(S) \ltimes \mathbb{R}^{S}$, where $\mathbb{R}^{S}$ denotes the space of functions on $S$, is obtained from BMSW by restricting the Weyl factor to be identified with the divergence of the vector field

$$
W^{\mathrm{bms}}=\frac{1}{2} \bar{D}_{A} Y^{A}
$$

The original BMS group $\operatorname{Conf}(S) \ltimes \mathbb{R}^{S}$ is obtained by fixing also the boundary metric to be the round 2-sphere metric

$$
\bar{q}_{A B}=\stackrel{\circ}{q}_{A B}
$$

and by demanding, in addition, that the vector field belongs to the conformal group of $S$

$$
\stackrel{\circ}{D}_{\langle A} Y_{B\rangle}=0,
$$

where $\stackrel{\circ}{D}$ is the covariant derivative of the spherical metric, so that (4.9) is preserved. The extended BMS group $\left(\operatorname{Conf}(S) \ltimes \mathbb{R}^{S}\right) \times \mathbb{R}^{S}$, which will be discussed in more details below, is obtained by imposing that sphere diffeomorphisms are conformal (4.10), but relaxing the Weyl condition (4.8) and introducing a field-dependent redefinition of the super-translation parameter $T$ in order to obtain an algebra. We come back to all these relations with more details in section 4.3 below.

\subsection{Boundary symmetry Lie algebra}

A remarkable consequence of the BMSW extension is that the residual diffeomorphisms generate a subalgebra, and not a subalgebroid, of the full diffeomorphism algebra of $\mathcal{I}$. This is not obvious from the start, since the BMSW vectors are manifestly field dependent. Therefore the commutator of the field transformations they generate is given by the modified Lie bracket (2.12), which in general yields an algebroid. To prove this point, we look at the leading order of the BMSW vector fields on $\mathcal{I}$. It is given by

$$
\bar{\xi}_{(\tau, Y)}:=\tau \partial_{u}+Y^{A} \partial_{A}-\dot{\tau} r \partial_{r},
$$

or equivalently

$$
\bar{\xi}_{(T, W, Y)}:=T \partial_{u}+Y^{A} \partial_{A}+W\left(u \partial_{u}-r \partial_{r}\right) .
$$

These vector fields are elements of the automorphism group of a line bundle $P \rightarrow \mathcal{I}$ over $\mathcal{I}$ that we call the scale bundle. The translation along the fiber of $P$ is given by the conformal rescaling operation and the asymptotic vector field is an element of its automorphism group, $\bar{\xi}_{(\tau, Y)}=\bar{\xi}_{(T, W, Y)} \in \operatorname{Aut}(P)$. At the infinitesimal level the conformal rescaling is implemented on $\mathcal{I}$ by the operator $r \partial_{r}$.

The two expressions (4.11), (4.12) give two different interpretations of the same vector field: in the first expression we see that $\tau$ labels linear time reparametrization while $\dot{\tau}$ 
labels a Weyl rescaling. In the second expression we see that $T$ labels super-translation, while $W$ labels a super-boost transformation which preserves the normal metric $2 \mathrm{~d} u \mathrm{~d} r$. Demanding that the boundary symmetry algebra is a Lie algebra, and not an algebroid, means that we have to impose that $\bar{\xi}_{(\tau, Y)}$ is field independent, i.e., $\delta \tau=\delta Y=0$. These conditions mean that

$$
\llbracket \bar{\xi}_{\left(\tau_{1}, Y_{1}\right)}, \bar{\xi}_{\left(\tau_{2}, Y_{2}\right)} \rrbracket=\left[\bar{\xi}_{\left(\tau_{1}, Y_{1}\right)}, \bar{\xi}_{\left(\tau_{2}, Y_{2}\right)}\right]_{\text {Lie }} .
$$

The vector fields $\bar{\xi}_{(\tau, Y)}$ are the generators of the boundary symmetry group. Their Lie commutators give

$$
\left[\bar{\xi}_{\left(\tau_{1}, Y_{1}\right)}, \bar{\xi}_{\left(\tau_{2}, Y_{2}\right)}\right]_{\text {Lie }}=\bar{\xi}_{\left(\tau_{12}, Y_{12}\right)}
$$

where

$$
\tau_{12}:=\tau_{1} \dot{\tau}_{2}-\tau_{2} \dot{\tau}_{1}+Y_{1}\left[\tau_{2}\right]-Y_{2}\left[\tau_{1}\right], \quad Y_{12}:=\left[Y_{1}, Y_{2}\right]_{\text {Lie }}
$$

We thus see that, in the $(\tau, Y)$ basis, the infinitesimal generators of the BMSW group at leading order in the Taylor expansion form a Lie algebra given by the semi-direct sum

$$
\text { bmsw := } \operatorname{diff}(S) \oplus \mathbb{R}_{u}^{S},
$$

with $\mathbb{R}_{u}^{S}$ denoting functions on the sphere $S$ which are linear in time with a bracket given by the Witt bracket. ${ }^{11}$ Quite remarkably, ${ }^{12}$ the bmsw symmetry algebra was shown to be the symmetry algebra preserving the so-called intrinsic structure ${ }^{13}$ of any finite null surface in [31]. This intrinsic structure can be understood as a thermal Carroll structure. This group also appears as the extended corner symmetry group of a 2 -sphere embedded in on a $3 \mathrm{~d}$ hypersurface [63]. The fact that the same symmetry group appears naturally at infinity, although surprising, is not a coincidence. We expect that asymptotic structure and asymptotic symmetry mirror similar structure at finite surfaces.

We can make this algebra even more explicit if one uses the explicit parametrization $\tau=T+u W$. Then we can rewrite (4.16) as a double semi-direct sum

$$
\text { bmsw }=\left(\operatorname{diff}(S) \oplus \mathbb{R}_{W}^{S}\right) \oplus \mathbb{R}_{T}^{S},
$$

where the first factor is the Weyl group parametrized by $W$, while the second factor is the super-translation group parametrized by $T$ (with $\delta T=\delta W=0$ ). The brackets are

$$
W_{12}=Y_{1}\left[W_{2}\right]-Y_{2}\left[W_{1}\right], \quad T_{12}=Y_{1}\left[T_{2}\right]-W_{1} T_{2}-\left(Y_{2}\left[T_{1}\right]-W_{2} T_{1}\right) .
$$

We see that $W$ transforms as a scalar under $\operatorname{diff}(S)$ while $T$ transforms as a weight-1 section of the scale bundle. A section $\Phi$ of the scale bundle is said to be of scale weight

\footnotetext{
${ }^{11} G^{S}$ refers to the set of maps from $S$ to the group $G . \mathbb{R}_{u}$ can also be identified with the subalgebra $\tau \partial_{u}$ of diffeomorphisms of $\mathbb{R}$ which are linear in $u$.

${ }^{12}$ We thank A. Speranza for bringing this result to our attention.

${ }^{13} \mathrm{An}$ intrinsic structure on a null surface $\mathcal{N}$ is an equivalence class of pairs $(n, \kappa)$ where $n=n^{a} \partial_{a}$ is a null generator of $\mathcal{N}$ and $\kappa$ is the null surface gravity. The equivalence relation is under rescaling of the null generator given by

$$
n \rightarrow e^{\sigma} n, \quad \kappa \rightarrow e^{\sigma}\left(\kappa+\mathcal{L}_{n} \sigma\right) .
$$
}

These transformations insure that the operator $\mathcal{L}_{n}+\kappa$ transforms tensorially. 
$s$ if it transforms under Weyl rescaling as $\Phi \rightarrow e^{-s W} \Phi$. This structure allows one to associate a notion of scale weight to fields on $\mathcal{I}$, something that has been considered in the literature $[48,90,102]$ and that will be explored elsewhere.

We can now formalize the construction (4.2) of the bulk vector field solution of the Bondi gauge condition as the construction of a map $\rho: \operatorname{Aut}(P) \rightarrow \Gamma(T M)$ given by (4.2). The BMSW vector fields $\xi_{(\tau, Y)}$ are the bulk extension of $\bar{\xi}_{(\tau, Y)}$ and they are given at leading orders by the asymptotic expansion

$$
\xi_{(\tau, Y)}=\rho\left(\bar{\xi}_{(\tau, Y)}\right)=\bar{\xi}_{(\tau, Y)}+\frac{1}{r} \xi_{\tau}^{1}+\frac{1}{r^{2}} \xi_{\tau}^{2}+o\left(r^{-2}\right)
$$

where

$$
\begin{aligned}
\xi_{\tau}^{1} & =-\partial^{A} \tau \partial_{A}+\frac{1}{2} \bar{\Delta} \tau r \partial_{r} \\
\xi_{\tau}^{2} & =\frac{1}{2} C^{A B} \partial_{B} \tau \partial_{A}-\frac{1}{2}\left(\bar{D}_{A} C^{A B} \partial_{B} \tau+\frac{1}{2} C^{A B} \bar{D}_{A} \partial_{B} \tau\right) r \partial_{r}
\end{aligned}
$$

The map $\rho$ gives an intertwiner between the standard Lie bracket and the field-depended modified Lie bracket (2.12). More precisely, thanks to the map $\rho$, the bulk vectors (4.19) form a faithful representation of the Lie algebra (4.16) for the modified Lie bracket (2.12). This follows from the property

$$
\llbracket \xi_{\left(\tau_{1}, Y_{1}\right)}, \xi_{\left(\tau_{2}, Y_{2}\right)} \rrbracket=\llbracket \rho\left(\bar{\xi}_{\left(\tau_{1}, Y_{1}\right)}\right), \rho\left(\bar{\xi}_{\left(\tau_{2}, Y_{2}\right)}\right) \rrbracket=\rho\left(\left[\bar{\xi}_{\left(\tau_{1}, Y_{1}\right)}, \bar{\xi}_{\left(\tau_{2}, Y_{2}\right)}\right]_{\text {Lie }}\right),
$$

which yields

$$
\llbracket \xi_{\left(\tau_{1}, Y_{1}\right)}, \xi_{\left(\tau_{2}, Y_{2}\right)} \rrbracket=\xi_{\left(\tau_{12}, Y_{12}\right)},
$$

with $\tau_{12}, Y_{12}$ given by (4.15). It follows that the bulk vectors in the $(\tau, Y)$ basis (4.19) also satisfy the Lie algebra (4.16).

A remark is in order. The construction of the algebra uses explicitly the preferred foliation of $\mathcal{I}$ introduced by the use of Bondi coordinates. For this reason, the right-hand side of (4.16) makes reference to the cross-sections $S$ defined at constant $u$. However, we expect that it is possible to use an intrinsic approach on $\mathcal{I}$ like in [31, 71], and define this algebra based on more general universal structure than the one associated to the BMS algebra. In this intrinsic approach, the only notion that is required is the fact that $\mathcal{I}$ is a fibration, without the need of choosing a specific foliation, and that this fibration is equipped with a thermal connection (see footnote 13). This will be investigated elsewhere.

\subsection{Extended BMS and generalized BMS as Lie sub-algebroids}

We now want to describe more precisely the relation between the BMSW group $(\operatorname{Diff}(S) \ltimes$ $\left.\mathbb{R}_{W}^{S}\right) \ltimes \mathbb{R}_{T}^{S}$ and the previous BMS groups. There are three such groups: the generalized BMS group Diff $(S) \ltimes \mathbb{R}_{T}^{S}[16,18]$, the extended BMS group $\left(\operatorname{Conf}(S) \ltimes \mathbb{R}_{T}^{S}\right) \times \mathbb{R}_{W}^{S}[48,90]$ and the original BMS group $\operatorname{Conf}(S) \ltimes \mathbb{R}_{T}^{S}[88,89]$. There is a key difference between BMSW and all the BMS groups: the BMS groups depend on a background structure held fixed, while the BMSW group does not involve any background structure besides the choice of foliation. 


\begin{tabular}{|c|c|c|c|}
\cline { 2 - 4 } \multicolumn{1}{c|}{} & $\xi_{T}$ & $\xi_{W}$ & $\xi_{Y}$ \\
\hline bmsw & super-translations & Weyl super-boost & Diff(S) sphere diffeomorphims \\
\hline generalized bms & super-translations & $\emptyset$ & super-Lorentz: rotations \& boosts \\
\hline extended bms & super-translations & super-Weyl & Conformal transformations \\
\hline
\end{tabular}

Table 2. Comparison of the extended, generalized BMS and BMSW generators. The generalized BMS generators are called super-Lorentz transformations, following [18, 100]. They are obtained from BMSW by fixing the Weyl rescaling in terms of the sphere diffeomorphism to preserve the metric scale. One usually calls super-rotations the area-preserving diffeomorphisms while the Lorentz super-boosts refer to transformations that are divergence-full, $D_{A} Y^{A} \neq 0$. In bmsw we have a finer group structure and the Weyl super-boosts are left free. Note that the Weyl super-boosts are, unlike Lorentz super-boosts, genuine boosts normal to the sphere. The Weyl super-boosts decouple in the extended BMS group.

On the one hand, all the BMS groups descend from BMSW, which can then be seen as a consistent merging of the previous extensions. On the other hand, there is a subtle point: to obtain the (original, extended or generalized) BMS algebras, one needs to consider field-dependent vector fields. This creates a potential issue that these sub-algebras are only sub-algebroids and not simply algebras. Having a Lie algebroid structure is not admissible if one wants to promote the classical symmetry algebra to a symmetry algebra of quantum gravity. One therefore needs to project back this algebroid onto an algebra. This is what we investigate and we show how to recover a Lie algebra structure in all three cases. The consequence of this reparametrization is that the representation of the sphere diffeomorphisms is fundamentally different for the BMS groups because it involves a mixing of the BMSW $\operatorname{diff}(S)$ and Weyl charges.

\subsubsection{Generalized bms}

The generalized bms algebra, or gbms for short, was first proposed by Campiglia and Laddha [16] as an asymptotic symmetry group to take into account the subleading soft theorem and it was extensively studied by Compere et al. in [18]. To obtain gbms from bmsw one has to redefine the Weyl parameter to be field dependent,

$$
W^{\text {gbms }}=\frac{1}{2} \bar{D}_{A} Y^{A} .
$$

This parametrization corresponds to a choice where the scale factor is fixed by the symmetry transformations

$$
\delta_{\xi \mathrm{gbms}} \sqrt{\bar{q}}=0 .
$$

With this choice, let us consider the asymptotic vector fields

$$
\bar{\xi}_{(T, Y)}^{\text {gbms }}:=\bar{\xi}_{\left(T, \frac{1}{2} \bar{D}_{A} Y^{A}, Y\right)},
$$

which become field dependent

$$
\delta \bar{\xi}_{(T, Y)}^{\mathrm{gbms}}=\frac{1}{2} Y[\delta \ln \sqrt{\bar{q}}]\left(u \partial_{u}-r \partial_{r}\right) .
$$




\begin{tabular}{|c|c|c|c|c|}
\cline { 2 - 5 } \multicolumn{1}{c|}{} & background structure & restriction & parametrisation & group \\
\hline bmsw & $\emptyset$ & $\emptyset$ & $(T, W, Y)$ & $\operatorname{Diff}(S) \ltimes_{0}\left(\mathbb{R}_{W}^{S} \ltimes \mathbb{R}_{T}^{S}\right)$ \\
\hline generalized bms & scale structure & $\delta \sqrt{q}=0$ & $\left(T, \frac{1}{2} D_{A} Y^{A}, Y\right)$ & $\operatorname{Diff}(S) \ltimes_{1 / 2} \mathbb{R}_{T}^{S}$ \\
\hline extended bms & conformal structure & $\delta\left[q_{A B}\right]=0$ & $\left(e^{\varphi} t, \frac{1}{2}\left(D_{A} Y^{A}-w\right), Y\right)$ & $\left(\operatorname{Conf}(S) \ltimes_{1 / 2} \mathbb{R}_{T}^{S}\right) \times \mathbb{R}_{W}^{S}$ \\
\hline
\end{tabular}

Table 3. Comparison of the extended, generalized BMS and BMSW group structure and parametrisation. The notation $\ltimes_{\alpha}$ denotes the action of diffeomorphims on densities of weight $\alpha$. $\mathbb{R}_{T}^{S}$ refers to super-translations and $\mathbb{R}_{W}$ to Weyl super-boost.

As a consequence, while the vectors $\xi_{(\tau, Y)}$ form a Lie algebra, the vectors $\bar{\xi}_{(T, Y)}^{\text {gbms }}$ form a Lie algebroid, unless we take the restriction that $\delta \ln \sqrt{\bar{q}}=0$, which is what is implemented for the generalized bms algebra [18]. This restriction insures that $\bar{\xi}^{\mathrm{gbms}}$ is field independent and therefore that the algebroid is an algebra.

The generalized bms Lie algebra bracket is

$$
\llbracket \bar{\xi}_{\left(T_{1}, Y_{1}\right)}^{\text {gbms }}, \bar{\xi}_{\left(T_{2}, Y_{2}\right)}^{\text {gbms }} \rrbracket=\bar{\xi}_{\left(T_{12}, Y_{12}\right)}^{\text {gbms }},
$$

with $\left(T_{12}, Y_{12}\right)$ given by

$$
\begin{aligned}
& Y_{12}^{A}=\left[Y_{1}, Y_{2}\right]_{\text {Lie }}^{A}, \\
& T_{12}=Y_{1}\left[T_{2}\right]-Y_{2}\left[T_{1}\right]+\frac{1}{2} T_{1} \bar{D}_{A} Y_{2}^{A}-\frac{1}{2} T_{2} \bar{D}_{A} Y_{1}^{A} .
\end{aligned}
$$

This algebra is such that $\operatorname{diff}(S)$ does not act on $W$, while its action on $T$ is the action on densities of weight $1 / 2$. In other words

$$
\text { gbms }=\left(\operatorname{diff}(S) \oplus_{1 / 2} \mathbb{R}_{T}^{S}\right),
$$

where $\oplus_{\alpha}$ denotes the action of diffeomorphism on densities of weight $\alpha$.

The fact that this is an algebra and not an algebroid stems from the fact that the scale factor $\sqrt{\bar{q}}$ is taken to be a background structure which is not part of the phase space and that the algebra action is consistent with the condition $\delta \sqrt{\bar{q}}=0=\delta_{\xi \text { gbms }} \sqrt{\bar{q}}$.

\subsubsection{Extended bms}

The extended bms algebra, denoted ebms and proposed by Barnich and Troessaert [48, 90], does not allow for general diffeomorphism but it allows for a Weyl rescaling of the metric. It is obtained from the bmsw algebra by introducing a background conformal structure ${ }^{14}$ associated with the round sphere metric $\stackrel{q}{q}$, and by imposing that ${ }^{15}$

$$
\bar{q}_{A B}=e^{2 \varphi} \stackrel{\circ}{q}_{A B}
$$

\footnotetext{
${ }^{14}$ That is an equivalence class $\left[q_{A B}\right]$ of metrics modulo rescaling $q_{A B} \sim e^{2 \varphi} q_{A B}$. By the fundamental theorem of Riemann surfaces a conformal structure is equivalent to a complex structure.

${ }^{15}$ Sometimes it is also considered the case where one chooses a north pole $n$ and a south pole $s$ on $S$ and introduces a flat space metric $q_{A B}^{\text {Flat }}$ on $S^{*}=S \backslash\{n, s\}$. This is possible since $S^{*}$ has the topology of the cylinder. In this case one can also consider imposing $\bar{q}_{A B}=e^{2 \varphi} q_{A B}^{\text {Flat }}$ on $S^{*}$. Such metrics are however singular on $S$.
} 
Preserving this background-dependent conditions requires modifying the definition of the Weyl factor. One imposes that

$$
W^{\mathrm{ebms}}=\frac{1}{2}\left(\bar{D}_{A} Y^{A}-w\right)
$$

and demands that it is $w$ which is now field independent $\delta w=0$. This choice of parametrization corresponds to transformations that preserve the sphere complex structure

$$
\delta_{\xi^{\mathrm{ebms}}} \sqrt{\bar{q}}=w \sqrt{\bar{q}}, \quad \delta_{\xi^{\mathrm{ebms}}} \varphi=\frac{1}{2} w .
$$

This creates a issue: the boundary vector field is then field dependent and we are at risk of creating an algebroid not an algebra, which would be not suitable for quantization. There is a way around it, that is to redefine also the super-translation generator as

$$
T^{\mathrm{ebms}}:=e^{\varphi} t,
$$

where $t$ is a field independent parameter, satisfying $\delta t=0$. The boundary vector field $\bar{\xi}_{(t, w, Y)}^{\text {ebms }}=\bar{\xi}_{\left(T^{\text {ebms }}, W^{\text {ebms }}, Y\right)}$ is still field dependent

$$
\delta \bar{\xi}_{(t, w, Y)}^{\mathrm{ebms}}=(\delta \varphi) T^{\mathrm{ebms}} \partial_{u}+Y[\delta \varphi]\left(u \partial_{u}-r \partial_{r}\right),
$$

nevertheless one can show that the extended bms algebroid is in fact a Lie algebra. The extended bms Lie algebra bracket is

$$
\llbracket \bar{\xi}_{\left(t_{1}, w_{1}, Y_{1}\right)}^{\mathrm{ebms}}, \bar{\xi}_{\left(t_{2}, w_{2}, Y_{2}\right)}^{\mathrm{ebms}} \rrbracket=\bar{\xi}_{\left(t_{12}, w_{12} Y_{12}\right)}^{\mathrm{ebbms}},
$$

with $[48,103]\left(t_{12}, w_{12}, Y_{12}\right)$ given by

$$
\begin{aligned}
Y_{12}^{A} & =\left[Y_{1}, Y_{2}\right]_{\text {Lie }}^{A}, \quad w_{12}=0, \\
t_{12} & =Y_{1}\left[t_{2}\right]-\frac{1}{2} t_{2} \stackrel{\circ}{D}_{A} Y_{1}^{A}-\left(Y_{2}\left[t_{1}\right]-\frac{1}{2} t_{1} \stackrel{\circ}{D}_{A} Y_{2}^{A}\right) .
\end{aligned}
$$

This means that the algebra is

$$
\text { ebms }=\left(\operatorname{Conf}_{\dot{q}}(S) \oplus_{1 / 2} \mathbb{R}_{T}^{S}\right) \oplus \mathbb{R}_{W}^{S} .
$$

The main feature of this algebra is the fact that the Weyl rescalings decouple from the conformal transformations and the super-translations. The Weyl parameter acts as a superselection parameter. Finally, the super-translations are acted upon by the conformal generators as density of weight $1 / 2$, in agreement with the generalized bms algebra.

Finally, the bms algebra

$$
\mathrm{bms}=\left(\operatorname{Conf}_{\stackrel{q}{\imath}}(S) \oplus_{1 / 2} \mathbb{R}_{T}^{S}\right)
$$

can be obtained either from the generalized bms algebra by restricting the diffeomorphisms to be conformal transformations or from the extended bms algebra by restricting the Weyl transformations to be trivial. 


\subsection{Action on the asymptotic phase space}

The asymptotic covariant phase space we define is parametrized by functionals of the metric $\Phi^{i}\left(g_{\mu \nu}\right)=\left(\bar{F}, M, \bar{\beta}, \bar{P}_{A}, \bar{U}_{A}, \bar{q}_{A B}, C_{A B}\right)$. We are interested in the behaviour of these functionals under the transformation $\delta_{(\tau, Y)} \Phi^{i}=\int \frac{\delta \Phi^{i}}{\delta g_{\mu \nu}} \mathcal{L}_{\xi_{(\tau, Y)}} g_{\mu \nu}$ generated by the BMSW group.

This can be found expanding the action of $\xi_{(\tau, Y)}$ on the metric in inverse powers of $r$,

$$
£_{\xi_{(\tau, Y)}} g_{\mu \nu}=£_{\bar{\xi}_{(\tau, Y)}} g_{\mu \nu}+\mathcal{L}_{\frac{1}{r} \xi_{1}+\frac{1}{r^{2}} \xi_{2}+o\left(r^{-2}\right)} g_{\mu \nu} .
$$

Accordingly, we can decompose the transformation of the functionals $\Phi^{i}$ as

$$
\delta_{(\tau, Y)} \Phi^{i}=\delta_{\bar{\xi}_{(\tau, Y)}} \Phi^{i}+\Delta_{\xi} \Phi^{i},
$$

where the homogeneous term $\delta_{\bar{\xi}_{(\tau, Y)}}$ is determined by $\mathcal{I}$-component $\bar{\xi}_{(\tau, Y)}$ of the Bondi vector fields (4.19), while the anomaly term $\Delta_{\xi}$ is determined by their bulk extension. ${ }^{16}$ An explicit calculation of the homogeneous part and the anomaly contribution yields ${ }^{17}$

$$
\begin{aligned}
\delta_{(\tau, Y)} \bar{q}_{A B}= & {\left[\tau \partial_{u}+\mathcal{L}_{Y}-2 \dot{\tau}\right] \bar{q}_{A B} } \\
\delta_{(\tau, Y)} C_{A B}= & {\left[\tau \partial_{u}+\mathcal{L}_{Y}-\dot{\tau}\right] C_{A B}-2 \bar{D}_{\langle A} \partial_{B\rangle} \tau } \\
\delta_{(\tau, Y)} N_{A B}= & {\left[\tau \partial_{u}+\mathcal{L}_{Y}\right] N_{A B}-2 \bar{D}_{\langle A} \partial_{B\rangle} \dot{\tau} } \\
\delta_{(\tau, Y)} M= & {\left[\tau \partial_{u}+\mathcal{L}_{Y}+3 \dot{\tau}\right] M+\left(\frac{1}{2} \bar{D}_{A} \dot{C}^{A B}+\partial^{B} \bar{F}\right) \partial_{B} \tau } \\
& +\frac{1}{4} N^{A B} \bar{D}_{A} \partial_{B} \tau+\frac{1}{4} C^{A B} \bar{D}_{A} \partial_{B} \dot{\tau} \\
\delta_{(\tau, Y)} \bar{P}_{A}= & {\left[\tau \partial_{u}+\mathcal{L}_{Y}+2 \dot{\tau}\right] \bar{P}_{A} } \\
& +3 M \partial_{A} \tau-\frac{1}{8} N_{B C} C^{B C} \partial_{A} \tau+\frac{1}{2}\left(C_{A}^{C} N_{B C}\right) \partial^{B} \tau \\
& +\frac{3}{4}\left(\bar{D}_{A} \bar{D}_{C} C_{B}{ }^{C}-\bar{D}_{B} \bar{D}_{C} C_{A}{ }^{C}\right) \partial^{B} \tau+\frac{1}{4} \partial_{A}\left(C^{B C} \bar{D}_{B} \bar{D}_{C} \tau\right) \\
& +\frac{1}{2} \bar{D}_{\langle A} \bar{D}_{B\rangle} \tau \bar{D}_{C} C^{B C}+C_{A B}\left(\bar{F} \partial^{B} \tau+\frac{1}{4} \partial^{B} \Delta \tau\right) .
\end{aligned}
$$

These expressions agree with the ones given in $[18,21]$ if we set $W=\frac{1}{2} \bar{D}_{A} Y^{A}$ and take into account the relation between the Barnich-Troessaert momentum $N_{A}$ and the canonical momentum $\bar{P}_{A}$ given by $N_{A}=\bar{P}_{A}+\partial_{A} \bar{\beta}$. We can also deduce that

$$
\begin{aligned}
\delta_{(\tau, Y)} \bar{F} & =\left[\tau \partial_{u}+\mathcal{L}_{Y}+2 \dot{\tau}\right] \bar{F}+\frac{1}{2} \bar{\Delta} \dot{\tau}, \\
\delta_{(\tau, Y)} \bar{\beta} & =\left[\tau \partial_{u}+\mathcal{L}_{Y}+2 \dot{\tau}\right] \bar{\beta}+\frac{1}{8} C^{A B} \bar{D}_{A} \partial_{B} \tau,
\end{aligned}
$$

\footnotetext{
${ }^{16}$ The term 'anomaly' refers to the fact that quantities like $C_{A B}$ or $M$ do not transform as sections of the scale bundle. The additional inhomogeneous terms come from the fact that they are components of a spacetime tensor. These are examples of the type of anomaly transformations defined in (2.3).

${ }^{17}$ We have included the time derivative term $\partial_{u}$ in the transformation for $\bar{q}_{A B}$ to highlight the overall structure, although our boundary conditions spelled in section 2.2 are such that $\partial_{u} \bar{q}_{A B}=0$.
} 
where the first equality follows from the transformation of $g_{u u}$ and the second equality follows from the transformation of $g_{u r}$. Finally the action of the transformations on $g_{u A}$ gives

$$
\delta_{(\tau, Y)} \bar{U}_{A}=\left(\tau \partial_{u}+\mathcal{L}_{Y}+\dot{\tau}\right) \bar{U}_{A}+\frac{1}{2}\left(4 \bar{F} \partial_{A} \tau+\partial_{A} \Delta \tau\right)+\frac{1}{2}\left(C_{A}^{B} \partial_{B} \dot{\tau}-N_{A}^{B} \partial_{B} \tau\right) .
$$

Recalling the relation $\bar{R}(\bar{q})=4 \bar{F}$, we see that the transformation $\delta_{(\tau, 0)} \bar{F}=2 \dot{\tau} \bar{F}+\frac{1}{2} \bar{\Delta} \dot{\tau}$ matches the infinitesimal conformal transformation of the $2 \mathrm{~d}$ Ricci scalar under the metric rescaling $\bar{q}_{A B} \rightarrow e^{-2 \dot{\tau}} \bar{q}_{A B}$. Similarly the variations (4.42b) and (4.43) can also be obtained through the on-shell identifications $(2.27 \mathrm{~b})$.

\subsection{Covariant functionals}

The transformation rules reported in the previous section have the general structure

$$
\delta_{(\tau, Y)} \mathcal{O}=\left[\tau \partial_{u}+\mathcal{L}_{Y}-s \dot{\tau}\right] \mathcal{O}+L_{\mathcal{O}}^{A} \partial_{A} \tau+\tilde{L}_{\mathcal{O}}^{A} \partial_{A} \dot{\tau}+Q_{\mathcal{O}}^{A B} \bar{D}_{A} \partial_{B} \tau+\tilde{Q}_{\mathcal{O}}^{A B} \bar{D}_{A} \partial_{B} \dot{\tau}
$$

The first term is the homogeneous transformation that involves the scale weight $s$ of the functional $\mathcal{O}$. All scale weights of the different functionals can be found by assigning scale weight $s\left(\mathrm{~d} s^{2}\right)=-2$, while $s(r)=s(\mathrm{~d} r)=-1$ and $s(u)=s(\mathrm{~d} u)=+1$ in the metric expansion, hence the scale weight of $\partial_{u}$ is -1 . Functionals that transform homogeneously are section of the scale bundle $P$. The inhomogeneous terms are of two types, $\left(L_{\mathcal{O}}^{A}, \tilde{L}_{\mathcal{O}}^{A}\right)$ which we call linear anomalies and terms $\left(Q_{\mathcal{O}}^{A B}, \tilde{Q}_{\mathcal{O}}^{A B}\right)$ which are the quadratic anomalies.

Requiring the absence of quadratic anomalies turns out to single out important quantities. A prime example of this is the time derivative of the news tensor, which transforms as $\delta_{(\tau, Y)} \dot{N}_{A B}=\left[\tau \partial_{u}+\mathcal{L}_{Y}+\dot{\tau}\right] \dot{N}_{A B}$, i.e., as a section of weight -1 of the scale bundle. A second example is the covariant mass aspect combination

$$
\mathcal{M}:=M+\frac{1}{8} N^{A B} C_{A B},
$$

whose transformation is

$$
\delta_{(\tau, Y)} \mathcal{M}=\left[\tau \partial_{u}+\mathcal{L}_{Y}+3 \dot{\tau}\right] \mathcal{M}+M^{A} \partial_{A} \tau, \quad M^{A}:=\frac{1}{2} \bar{D}_{B} N^{A B}+\partial^{A} \bar{F} .
$$

The quantity $M^{A}$ appearing here is itself free of quadratic anomalies. In fact, an explicit calculation gives

$$
\delta_{(\tau, Y)} M^{A}=\left[\tau \partial_{u}+\mathcal{L}_{Y}+4 \dot{\tau}\right] M^{A}+\frac{1}{2} \dot{N}^{A B} \partial_{B} \tau,
$$

where we used that $\left[\partial_{A}, \bar{\Delta}\right] \dot{\tau}=-\frac{1}{2} R \partial_{A} \dot{\tau}$. This shows that if the vacuum structure $M^{A}=$ $0=\dot{N}_{A B}$ is satisfied, then the covariant mass aspect $\mathcal{M}$ transforms homogeneously. As a consequence, the manifold of flat vacua can be defined by the conditions

$$
\mathcal{M}=0, \quad \dot{N}_{A B}=0, \quad M^{A}=0 .
$$

These conditions define an orbit of the BMSW group. 
Demanding no quadratic anomaly in the transformation rule for the momentum, ${ }^{18}$ we are led to the covariant momentum aspect

$$
\mathcal{P}_{A}:=\bar{P}_{A}-2 \partial_{A} \bar{\beta}-\frac{1}{2} C_{A B} \bar{U}^{B} .
$$

As shown in [56], this momentum aspect is the one with the simplest transformation under super-translations. It is also the unique one with no quadratic anomaly, therefore it possesses the simplest transformation under the BMSW group.

There is a further, independent reason to single out these covariant quantities: they are the leading order contributions to the Weyl scalars. As shown explicitly in appendix D, the covariant momentum aspect is the leading order of the Weyl scalar $\psi_{1}$, the covariant mass $\mathcal{M}$ of (the real part of) $\psi_{2}$, the vector shift $M_{A}$ of $\psi_{3}$, and $\dot{N}_{A B}$ of $\psi_{4}$.

\subsection{On the definition of (angular) momentum}

Our analysis has led us to consider two different momentum charge aspects, $\bar{P}_{A}$ and $\mathcal{P}_{A}$. They appear in the expansion of $U^{A}(2.26 \mathrm{c})$ as

$$
\begin{aligned}
U^{A} & =\frac{1}{r^{2}} \bar{U}^{A}-\frac{2}{3 r^{3}} \bar{q}^{A B}\left(\bar{P}_{B}+C_{B C} \bar{U}^{C}+\partial_{B} \bar{\beta}\right), \\
& =\frac{1}{r^{2}} \bar{U}^{A}-\frac{2}{3 r^{3}} \bar{q}^{A B}\left(\mathcal{P}_{B}+\frac{3}{2} C_{B C} \bar{U}^{C}+3 \partial_{B} \bar{\beta}\right) .
\end{aligned}
$$

The momentum aspect $\bar{P}_{A}$ is the canonical charge generating sphere diffeomorphisms for all the bms algebra extensions. It is important to appreciate that these charges are defined at the finite $u=0$ sphere (see (6.8) below for the $u=c s t$ shift of this momentum which acquires an extra term linear in $u$ ). The fact that $\bar{P}_{A}$ is the canonical charge aspect of finite cuts is consensual across different approaches in the literature, and its expression coincides in Ashtekar-Streubel [71], Wald-Zoupas [46], Barnich-Troessaert [21], FlanaganNichols [17]. For this reason we refer to it as the canonical momentum. On the other hand, the covariant momentum aspect $\mathcal{P}_{A}$ appears in Hawking-Perry-Strominger [105] and Compere-Fiorucci-Ruzziconi [51] as charges attached to the limiting spheres at $u= \pm \infty$. In addition to the property that this momentum possesses no quadratic anomaly, there is also the fact that it vanishes at timelike infinity, i.e. when $u \rightarrow+\infty$. More generally, it vanishes for vacuum spacetime.

Even though the covariant and canonical momentum charges are unique in the literature, the parametrization (4.50) of the metric coefficient $U^{A}$ in terms of (angular) momentum, ${ }^{19}$ usually denoted $N^{A}$, is far from universal and distinct from the definition of physical charges. While the latter are clearly more relevant and matter more, to help the reader to navigate the different conventions we summarize here different parametrization used in the literature. To rationalize the discussion, we consider as in [56] a two-parameter family

$$
P_{A}^{(a, b)}:=\bar{P}_{A}-\frac{a}{2} C_{A B} \bar{U}^{B}-2 b \partial_{A} \bar{\beta} .
$$

\footnotetext{
${ }^{18}$ The explicit formula for the transformation will appear in a forthcoming work [104].

${ }^{19}$ We explained in the Introduction why we prefer the name momentum as opposed to angular momentum.
} 
The covariant momentum is $\mathcal{P}_{A}=P_{A}^{(1,1)}$ and the canonical momentum is $\bar{P}_{A}=P_{A}^{(0,0)}$. Most of the literature oscillates between these two parameterization of the metric coefficient: the canonical class, which uses $N_{A}=\bar{P}_{A}$, includes Ashtekar-Streubel [71], WaldZoupas [46], while the covariant class, which uses $N_{A}=\mathcal{P}_{A}$, includes Yau et al. [67], Flanagan-Nichols [17], Strominger, Hawking-Perry-Strominger [105]. There are a few authors that consider a different parametrization, that include Barnich-Troessaert [48], Compere-Fiorucci-Ruzziconi [18] and Mädler-Winicour [6] for $(a, b)=(0,-1 / 2)$.

Let us note that this discussion was for the BMS momenta, which generate diffeomorphisms combined with a rescaling that preserves the metric determinant. The BMSW canonical momentum, which generates a pure $\operatorname{Diff}(S)$, is shifted with respect to the BMS momenta and given by $P_{A}^{(0,-1)}$, see (2.43).

\subsection{Vacuum structure}

As we have seen, the non-radiative sector of the theory is characterized by the conditions $\dot{N}_{A B}^{\mathrm{vac}}=0$, and $M_{A}^{\mathrm{vac}}=0$. These are solved ${ }^{20}$ by $N_{A B}^{\mathrm{vac}}=T_{A B}(\bar{q})$ where $T_{A B}(\bar{q})$ is the Liouville energy-momentum tensor $[18,69]$ associated with the metric and defined by

$$
\bar{D}^{A} T_{A B}(\bar{q})=-\frac{1}{2} \partial_{B} \bar{R}
$$

It vanishes for the round metric $\stackrel{9}{\text {. }}$

We can also understand the vacuum sector as an orbit of the BMSW group (see [90, 106] for an orbit analysis of the original BMS group). To do so we introduce the BMSW group elements $^{21}$

$$
g_{(T, W, Y)}=e^{\delta_{T}} e^{\delta_{W}} e^{\delta_{Y}} .
$$

Their action on the gravity phase space is given by exponentiation ${ }^{22}$ of the infinitesimal action (4.41a), (4.41b), (4.41d), (4.42a) as follows:

$$
\begin{aligned}
g_{(T, W, Y)} \cdot \bar{q}_{A B}= & e^{\mathcal{L}_{Y}}\left[e^{-2 W} \bar{q}_{A B}\right], \\
g_{(T, W, Y)} \cdot N_{A B}(u)= & e^{\mathcal{L}_{Y}}\left[N_{A B}\left(e^{W}(u+T)\right)-2\left(\bar{D}_{\langle A} \partial_{B\rangle} W+\partial_{\langle A} W \partial_{B\rangle} W\right)\right], \\
g_{(T, W, Y)} \cdot C_{A B}(u)= & e^{\mathcal{L}_{Y}}\left[e^{-W} C_{A B}\left(e^{W}(u+T)\right)-2\left(\bar{D}_{\langle A} \partial_{B\rangle} T+2 \partial_{\langle A} W \partial_{B\rangle} T\right)\right. \\
& \left.-2(u+T)\left(\bar{D}_{\langle A} \partial_{B\rangle} W+\partial_{\langle A} W \partial_{B\rangle} W\right)\right], \\
g_{(T, W, Y)} \cdot \bar{R}(\bar{q})= & e^{\mathcal{L}_{Y}}\left[e^{2 W}(\bar{R}(\bar{q})+2 \bar{\Delta} W)\right] .
\end{aligned}
$$

The vacua $|T, W, Y\rangle=\hat{g}_{(T, W, Y)}|0\rangle$ are labelled by a BMSW group element. In particular, starting from Minkowski spacetime, we see that the orbit of the BMSW group element $g_{(T, W, 0)}$ generates a non-zero value for the news tensor of the vacua given by

$$
N_{A B}^{\mathrm{vac}}=T_{A B}\left(e^{-2 W} \stackrel{\circ}{q}\right)=-2\left(\bar{D}_{\langle A} \partial_{B\rangle} W+\partial_{\langle A} W \partial_{B\rangle} W\right) .
$$

\footnotetext{
${ }^{20}$ Once one imposes the asymptotic Einstein equation $\bar{R}(\bar{q})=4 \bar{F}$.

${ }^{21}$ We do not use the $\tau$ parametrization which is more involved as $g_{\tau}=e^{\delta_{\tau}}=g_{T\left(e^{W}-1\right) / W, W}$.

${ }^{22}$ For instance
}

$$
g_{(T, W, Y)} \cdot q_{A B}=e^{\mathcal{L}_{Y}}\left[e^{\delta_{T}} e^{\delta_{W}} q_{A B}\right]=e^{\mathcal{L}_{Y}} e^{-2 W}\left[e^{\delta_{T}} q_{A B}\right]=e^{\mathcal{L}_{Y}} e^{-2 W} e^{T \partial_{u}} q_{A B} .
$$


Alternatively, it is well known that a generic metric $\bar{q}$ on the sphere can be obtained by the action of $g_{(T, W, Y)}$ on the round metric $\stackrel{\circ}{q}$. The kernel of this action is $\mathrm{SU}(2) \ltimes \mathbb{R}^{S}$, so we can also label the vacuum by a triple $\left(\bar{q}_{A B}, T, g\right)$, where $T$ is a super translation parameter and $g$ is an $\mathrm{SU}(2)$ group element.

The construction of the BMSW group presented so far applies to any formulation of gravity. We now want to show that the BMSW can be given a canonical formulation in terms of charges, defined using covariant phase space methods, and a suitable renormalization procedure along the lines explained in section 2. The next section will show that one can bypass the problem of integrability of the Hamiltonians, and work uniquely using the Noether charges. This is enough to recover the full BMSW algebra via a centerless bracket at any cross-section of scri, and furthermore to prove that the EEs themselves can be understood as the requirement that this algebra is represented in the phase space. To take these next steps, the choice of a specific Lagrangian and its fundamental variables is a priori necessary. In the following, we will specialize to the use of tetrad variables and the Einstein-Cartan formulation, for reasons that we now explain.

\section{Tetrad variables}

General relativity can be formulated using either the metric or the tetrad as fundamental variables. Both formulations are free of Lagrangian and symplectic anomalies, and the formalism developed in [44] and summarized in section 2 can be equally applied to both. In this paper, we choose to work in tetrad variables. This option is less common in the literature, but there are compelling reasons to do so, and indeed we would like to advocate that the study of boundary symmetries of gravity is better done in tetrad variables. In addition to the general advantages of working with tetrads, like the technical simplifications of working with forms, and better treatment of matter coupling and first-order version of the action principle, there are specific advantages in terms of covariant phase space methods. These include again simpler expressions to manipulate, but also removing the need of subtraction terms at spatial infinity [107], and a convenient framework for isolated and dynamical horizons, e.g. $[108,109]$. More relevant to the study of future null infinity is the recent result that tetrad variables give access to non-vanishing dual BMS charges [110-112].

When using tetrad variables, there is an important aspect to take into account: the additional gauge freedom of internal Lorentz transformations. This leads to internal Lorentz charges that are absent in the metric formalism, and a priori different covariant phase spaces. The differences show up for instance in the formulas for the quasi-local charges, which been investigated in $[81,113]$ and [38-40]; see also [107, 114-117] for previous related work. It is known that equivalence of the charges can be restored for isometries using the Kosmann derivative [114-116] (see discussion in [81]), but for asymptotic symmetries is it not always the case $[111,112]$ : at null infinity the standard charges are the same but not the dual ones, thus offering a set-up to recover known BMS results, while at the same time accessing the dual sector. The exact equivalence can be obtained for all charges including arbitrary diffeomorphisms if one works with a dressed symplectic potential [81, 113] (see also $[87,118])$. As explained in $[38,39]$, this choice of potential can be uniquely singled out adding a suitable boundary Lagrangian (see also [119, 120]). 
Recall from the Introduction that our approach is to focus on Noether charges, and be able to reproduce the algebra of all diffeomorphisms preserving the boundary conditions. In this perspective, the internal Lorentz charges are a key component of the corner symmetry group at finite distance, and are also in general non-vanishing at null infinity. While this might seem surprising at first, a moment of reflection shows that this charge contribution is indeed necessary if we are to fix an internal gauge for the frame field which is not preserved by the action of Bondi diffeomorphisms. In fact, we are going to show that the nonvanishing contribution of the internal Lorentz symmetry to the Noether charges is crucial in order to correctly recover the asymptotic Einstein's equations at null infinity.

\subsection{Charges and fluxes}

Let us briefly recall the explicit formulas of the Einstein-Cartan-Holst formulation and its symplectic structure that we will need below. The Lagrangian is

$$
L=\frac{1}{2} \Sigma_{I J} \wedge F^{I J}(\omega),
$$

with $\Sigma_{I J}=P_{I J K L} e^{K} \wedge e^{L}, P_{I J K L}=\frac{1}{2} \epsilon_{I J K L}+\frac{1}{\gamma} \eta_{I[K} \eta_{L] J}$ where $\gamma$ is the Barbero-Immirzi or simply Immirzi parameter, and the curvature is $F_{I J}(\omega)=\mathrm{d} \omega_{I J}+\frac{1}{2}[\omega, \omega]_{I J}$. The symplectic potential is given by

$$
\theta=\frac{1}{2} \Sigma_{I J} \wedge \delta \omega^{I J}
$$

The action of diffeomorphisms on the phase space variables is given by

$$
\delta_{\xi} \Sigma_{I J}=£_{\xi} \Sigma_{I J}=\mathrm{d}\left(\iota_{\xi} \Sigma_{I J}\right)+\iota_{\xi} \mathrm{d} \Sigma_{I J}, \quad \delta_{\xi} \omega^{I J}=£_{\xi} \omega^{I J}=\mathrm{d}_{\omega}\left(\iota_{\xi} \omega^{I J}\right)+\iota_{\xi} F^{I J} .
$$

The associated Noether current and charge aspect are

$$
I_{\xi} \theta-\iota_{\xi} L \hat{=} \mathrm{d} q_{\xi}, \quad q_{\xi}=\frac{1}{2} \Sigma_{I J} \iota_{\xi} \omega^{I J} .
$$

We used the vacuum Einstein's equations $P_{I J K L}\left(e^{J} \wedge F^{K L}\right) \hat{=} 0$ and $\mathrm{d}_{\omega} \Sigma^{I J} \hat{=} 0$. Since there is no anomaly, the Noetherian flux is given by

$$
\begin{aligned}
\mathcal{F}_{\xi} & =\iota_{\xi} \theta+q_{\delta \xi} \\
& =\frac{1}{2} \iota_{\xi}\left(\Sigma_{I J} \wedge \delta \omega^{I J}\right)+\frac{1}{2} \Sigma_{I J} \wedge \iota_{\delta \xi} \omega^{I J} \\
& =\frac{1}{2} \iota_{\xi} \Sigma_{I J} \wedge \delta \omega^{I J}+\frac{1}{2} \delta\left(\Sigma_{I J} \iota_{\xi} \omega^{I J}\right)-\frac{1}{2} \delta \Sigma_{I J} \iota_{\xi} \omega^{I J} .
\end{aligned}
$$

The action of gauge transformations on the phase space is given by

$$
\delta_{\lambda} \Sigma_{I J}=[\Sigma, \lambda]_{I J}, \quad \delta_{\lambda} \omega^{I J}=\mathrm{d}_{\omega} \lambda^{I J} .
$$

The associated Noether current and charge aspect are given on-shell by

$$
I_{\lambda} \theta \hat{=} \mathrm{d} q_{\lambda}, \quad q_{\lambda}=\frac{1}{2} \Sigma_{I J} \lambda^{I J},
$$

while the Noetherian flux is simply

$$
\mathcal{F}_{\lambda}=q_{\delta \lambda} .
$$




\section{$5.2 \quad$ Adapted tetrad}

To treat the case of null infinity, it is best to work with a tetrad in doubly-null form, with internal metric $\eta_{01}=-1=-\eta_{23}$ and complex dyad on the sphere. ${ }^{23}$ To avoid the risk of confusion, we distinguish the coframe and frame with a hat, $e^{I}=e_{\mu}^{I} \mathrm{~d} x^{\mu}$ and $\hat{e}_{I}=\hat{e}_{I}^{\mu} \partial_{\mu}$. We follow [111] and choose the following tetrad adapted to the foliation defined by the Bondi coordinates,

$$
\begin{array}{lll}
e^{0}=e^{2 \beta} \mathrm{d} u, & e^{1}=\mathrm{d} r+F \mathrm{~d} u, & e^{i}=r E_{A}^{i}\left(\mathrm{~d} \sigma^{A}-U^{A} \mathrm{~d} u\right), \\
\hat{e}_{0}=e^{-2 \beta}\left(\partial_{u}-F \partial_{r}+U^{A} \partial_{A}\right), & \hat{e}_{1}=\partial_{r}, & \hat{e}_{i}=\frac{1}{r} E_{i}^{A} \partial_{A} .
\end{array}
$$

Here $i=2,3$ are the internal indices of the dyad $E_{i}^{A}$ on the sphere, with inverse $E_{i}^{A}$ and related to the $2 \mathrm{~d}$ metric by $q_{A B}=E_{A}^{i} E_{B}^{j} \eta_{i j}$. The dyad is complex with the doubly-null choice, and real if we take $\eta_{i j}=\delta_{i j}$. This choice of tetrad is characterized by the fact that $\hat{e}_{i}$ are tangent to the sphere and by the fact that $\hat{e}_{1}=\partial_{r}$. This eliminates the null rotations around $\hat{e}_{0}$ and $\hat{e}_{1}$ and the boost transformations $\left(e^{0}, e^{1}\right) \rightarrow\left(\lambda e^{0}, \lambda^{-1} e^{1}\right)$. The only internal gauge freedom left is the tangential frame rotation $\delta_{\theta} \hat{e}^{i}=i \theta \epsilon^{i j} \eta_{j k} \hat{e}^{k}$. It can be fixed picking a specific frame or equivalently a $2 \mathrm{~d}$ spinor on the sphere $z_{A}$ and demanding that $E_{A}^{2}=z_{A}$ and $E_{A}^{3}=\bar{z}_{A}$. This tetrad follows the original Newman-Unti adapted tetrad [5] in taking the first null vector to be the tangent to the null geodesics, but with the difference that the second null vector is not parallel transported along $l$, but chosen so that the dyad is tangent to the sphere, or in other words adapted to the $2+2$ foliation defined by $(u, r)$. See [121] for a comparison of the various choices in the literature and their respective gauge fixings.

In terms of the frames, the vectors $l$ and $t$ read

$$
l=\hat{e}_{1}, \quad t=e^{2 \beta} \hat{e}_{0}+F \hat{e}_{1},
$$

and the torsionless spin connection $\omega_{\mu}^{I J}=e_{\nu}^{I} \nabla_{\mu} e^{\nu J}$ can be nicely written in terms of the geometric quantities of section 3 (see also [111]),

$$
\begin{aligned}
& \omega_{10}=F^{\prime} \mathrm{d} u+\frac{1}{r} \eta_{A}\left(\mathrm{~d} \sigma^{A}-U^{A} \mathrm{~d} u\right)+2 \beta^{\prime}(\mathrm{d} r+F \mathrm{~d} u) \\
& \omega_{1 i}=E_{i}^{A}\left[\frac{e^{2 \beta}}{r^{2}}\left(\eta_{A}-2 r \partial_{A} \beta\right) \mathrm{d} u-S_{A B}\left(d \sigma^{B}-U^{A} \mathrm{~d} u\right)\right] \\
& \omega_{0 i}=-E_{i}^{A}\left[\frac{1}{r} \partial_{A} F \mathrm{~d} u-e^{-2 \beta}\left(F S_{A B}-K_{A B}\right)\left(\mathrm{d} \sigma^{B}-U^{B} \mathrm{~d} u\right)+\frac{1}{r^{2}} \eta_{A}(\mathrm{~d} r+F \mathrm{~d} u)\right], \\
& \omega_{i j}=\left(E_{[i}^{A} \dot{E}_{j] A}+E_{[i}^{A} E_{j] B} D_{A} U^{B}\right) \mathrm{d} u+\left(E_{[i}^{A} E_{j] A}^{\prime}\right) \mathrm{d} r+w_{i j A} \mathrm{~d} \sigma^{A}
\end{aligned}
$$

In the last expression, $w_{i j A}=E_{[i}^{B} D_{A} E_{j] B}$ is the sphere's spin connection associated to the dyad $E_{A}^{i}$, and satisfies $\partial_{[A} E_{B]}^{i}+w_{j[A}^{i} E_{B]}^{j}=0$.

\footnotetext{
${ }^{23}$ This has the advantage of making results from the Newman-Penrose formalism accessible, but all formulas presented in this paper immediately extend to a real dyad and $\eta_{22}=\eta_{33}=1$.
} 
For the expansion of the tetrad at future null infinity we use the expressions (2.26), which we complement with the corresponding one for the dyad,

$$
E_{A}^{i}=\bar{E}_{A}^{i}+\frac{1}{2 r} C_{A}^{B} \bar{E}_{B}^{i}+\frac{1}{16 r^{2}} \bar{E}_{A}^{i} C_{B C} C^{B C}+o\left(r^{-2}\right) .
$$

Here $\bar{q}_{A B}=\bar{E}_{A}^{i} \bar{E}_{B}^{j} \eta_{i j}$, and we used the identity (B.13) to simplify the $r^{-2}$ term.

\subsection{Residual gauge transformations}

The general transformation of the tetrad is a linear combination of diffeomorphisms and internal Lorentz transformations,

$$
\delta_{(\xi, \lambda)} e_{\mu}^{I}=£_{\xi} e_{\mu}^{I}-\lambda^{I}{ }_{J} e_{\mu}^{J} .
$$

We have already determined the BMSW vectors fields which preserve the coordinate gauge and metric boundary conditions. When working with tetrads we also need to study which internal gauge transformations preserve the adapted form (5.9), in particular the gaugefixing conditions

$$
e_{A}^{0}=0, \quad e_{r}^{0}=0, \quad e_{A}^{1}=0 .
$$

Requiring that (5.13) preserves these conditions fixes uniquely five gauge parameters in terms of the BMSW vectors $\xi$,

$$
\begin{aligned}
\lambda_{\xi}^{0 i} & =\frac{e^{2 \beta}}{r} E^{i A} \partial_{A} \xi^{u} \\
\lambda_{\xi}^{1 i} & =\frac{1}{r} E^{i A}\left(\partial_{A} \xi^{r}+F \partial_{A} \xi^{u}\right), \\
\lambda_{\xi}^{01} & =\partial_{r} \xi^{r}=\partial_{r}\left(\frac{r}{2} D_{A}\left(I^{A B} \partial_{B} \tau\right)+\frac{r}{2} U^{A} \partial_{A} \tau-r \dot{\tau}\right) \\
& =-\dot{\tau}+\frac{1}{4 r^{2}} \bar{D}_{A}\left(C^{A B} \partial_{B} \tau\right)-\frac{1}{2 r^{2}} \bar{U}^{A} \partial_{A} \tau+o\left(r^{-3}\right),
\end{aligned}
$$

while $\lambda_{\xi}^{i j}$ is kept free. The last term will be the most important to us, it shows that the Weyl boost diffeomorphism needs to be accompanied by a internal boost transformation in the plane normal to the sphere. The strength of this internal boost being given by the boost factor $\partial_{r} \xi^{r}$. The action of the vector fields (4.19) on the frame fields is then

$$
\delta_{\xi} e_{\mu}^{I}=£_{\xi} e_{\mu}^{I}-\lambda_{\xi}^{I} e_{\mu}^{J} .
$$

The explicit asymptotic expansion of the gauge parameters $\lambda_{\xi}^{01}$ corresponding to $\xi_{T}, \xi_{W}, \xi_{Y}$ is given in appendix A. This expansion is relevant for the construction of Noether charges and fluxes.

\section{Noether charges: energy, Weyl and momentum}

As a consequence of (5.16), we see that it is not possible to study the asymptotic diffeomorphism charges without simultaneously including the effect of the internal charges. ${ }^{24}$ From

\footnotetext{
${ }^{24}$ This point was already made in [122], however there it had no consequences because they were restricting to the original BMS.
} 
the definition of Noether charge (2.8), and using the charge aspects associated to the diffeomorphism action (5.16), which combines (5.4) with an internal Lorentz symmetry (5.7), one gets

$$
Q_{\xi}=r^{2} \int_{S} \sqrt{q}\left(\iota_{\xi} \omega^{01}+\lambda_{\xi}^{01}\right) .
$$

In our present analysis, we are not going to include the dual super-translation charges discovered in $[110,111]$, meaning that we set the Immirzi parameter $1 / \gamma \rightarrow 0$. As revealed in [39], this limit kills the internal Lorentz rotations, but it still gives us access to the internal Lorentz boosts, namely to the $\lambda_{\xi}^{01}$ component of the Noether charge (5.7).

We introduce a basis with elements associated to a single parameter and we simply denote $\xi_{T}:=\xi_{(T, 0,0)}, \xi_{W}:=\xi_{(0, W, 0)}$ and $\xi_{Y}:=\xi_{(0,0, Y)}$. The expansion (4.19) for those vectors gives

$$
\begin{aligned}
\xi_{T} & =T \partial_{u}-\frac{1}{r} \partial^{A} T \partial_{A}+\frac{1}{2}\left(\bar{\Delta} T-\frac{1}{2 r} \bar{D}_{A}\left(C^{A B} \partial_{B} T\right)+\frac{1}{r} \bar{U}^{A} \partial_{A} T\right) \partial_{r}+o\left(r^{-1}\right), \\
\xi_{W} & =u \xi_{T=W}-W r \partial_{r} \\
\xi_{Y} & =Y^{A} \partial_{A} .
\end{aligned}
$$

Note that in this basis the Weyl transformation $\xi_{W}$ is a radial dilation with parameter $W$ followed by $u$ times a super-translation with parameter $T=W$.

We specialize the vector field $\xi$ to be, respectively, the super-translation generator $\xi_{T}$ (6.2a), the Weyl super-boost transformation $\xi_{W}(6.2 \mathrm{~b})$, and the sphere diffeomorphisms $\xi_{Y}$ (6.2c). This yields the following leading order terms ${ }^{25}$

$$
\begin{aligned}
Q_{T} & =\int_{S} \sqrt{\bar{q}} T\left(M-\frac{1}{2} \bar{D}_{A} \bar{U}^{A}\right) \\
Q_{W} & =\int_{S} \sqrt{\bar{q}} W\left[-r^{2}+4 \bar{\beta}+u\left(M-\frac{1}{2} \bar{D}_{A} \bar{U}^{A}\right)\right], \\
Q_{Y} & =\int_{S} \sqrt{\bar{q}} Y^{A}\left(-r \bar{U}_{A}+\bar{P}_{A}+2 \bar{D}_{A} \bar{\beta}\right) .
\end{aligned}
$$

One important subtlety is that the bmsw $\operatorname{Diff}(S)$ or sphere diffeomorphism charge is not the same as the gbms super-Lorentz charge appearing in [18]. This follows from the relation (4.25) which states that a gbms vector field is a metric dependent bmsw vector field. Explicitly, this means that we can write the gbms vector fields as

$$
\xi_{T}^{\mathrm{gbms}}=\xi_{T}, \quad \xi_{Y}^{\mathrm{gbms}}=\xi_{Y}+\xi_{W=\frac{1}{2} \bar{D}_{A} Y^{A}}
$$

The main point here is that a gbms super-Lorentz transformation is the sum of a bmsw sphere diffeomorphism transformation plus a Weyl super-boost. In particular, this implies that the gbms super-translation and super-Lorentz charges are given by

$$
Q_{T}^{\mathrm{gbms}}=Q_{T}, \quad Q_{Y}^{\mathrm{gbms}}=Q_{Y}+Q_{W=\frac{1}{2} \bar{D}_{A} Y^{A}}
$$

\footnotetext{
${ }^{25}$ All the charge expressions are given modulo $o(1)$.
} 
This means that the finer structure of the BMSW group allows us to cleanly disentangle the effect of a sphere diffeomorphism from the effect of a Weyl boost. These two effects are colluded in the original and generalized BMS groups. This observation is essential when discussing the momentum Noetherian flux in the next section. In particular, the $\operatorname{Diff}(S)$ or sphere diffeomorphism flux is trivial for the BMSW group, as expected for a corner transformation that do not translate the sphere [38], while it is not for the generalized BMS group, simply because a gbms super-Lorentz includes a Weyl boost. If one writes the gbms super-Lorentz charge explicitly one gets the leading asymptotics

$$
Q_{Y}^{\text {gbms }}=\int_{S} \sqrt{\bar{q}} Y^{A}\left[-r \bar{U}_{A}+\bar{P}_{A}-\frac{u}{2} \bar{D}_{A}\left(M-\frac{1}{2} \bar{D}_{B} \bar{U}^{B}\right)\right] .
$$

This shows as promised that $\bar{P}_{A}$ is the finite and $u$-independent component of the BMS momentum aspect. This justifies a posteriori our parametrization.

\section{Noetherian fluxes: energy, Weyl and momentum}

In this section, we provide the asymptotic expressions of the Noetherian fluxes associated to the BMSW vector fields, leaving all details to appendix B. From the definition of Noetherian flux (2.9) and recalling that the Einstein-Cartan formulation is anomaly-free, one gets the expression

$$
\mathcal{F}_{\xi}:=\int_{S}\left(\iota_{\xi} \theta+q_{\delta \xi}+q_{\delta \lambda_{\xi}}\right)
$$

where the first contribution is the symplectic potential flux associated to diffeomorphisms (5.5), supplemented by the contribution due to the field-dependence of the vector field $\xi$ and the internal Lorentz symmetry (5.8). It is instructive to split the Noetherian flux in its three contributions and analyze their structure and properties. The symplectic potential flux is

$$
\mathcal{F}_{\xi}^{\theta}:=\int_{S} \iota_{\xi} \theta=\int_{S} \sqrt{q} e^{2 \beta} r^{2}\left(\xi^{r} \theta^{u}-\xi^{u} \theta^{r}\right)
$$

where $\theta=\theta^{\mu} \epsilon_{\mu}$, with $\epsilon_{\mu}=\iota_{\partial_{\mu}} \epsilon$ and $\epsilon=e^{2 \beta} r^{2} \sqrt{q} \mathrm{~d} u \mathrm{~d} r \mathrm{~d} \sigma^{2}$ is the 4-volume form - see appendix B for details. The fact that it depends only on the components $\left(\xi^{u}, \xi^{r}\right)$ means that it vanishes if $\xi$ is tangential to the sphere, as it is to be expected since the tangential diffeomorphisms have generators trivially integrable.

Let us analyse separately the expressions of the temporal and radial components. ${ }^{26}$ For the temporal flux, we have

$$
\begin{aligned}
-\sqrt{q} e^{2 \beta} r^{2} \theta^{r} & =r \sqrt{q} E_{i}^{A}\left(e^{2 \beta} \delta \omega_{A 0}{ }^{i}-F \delta \omega_{A 1}{ }^{i}\right)+r^{2} \sqrt{q}\left(\delta \omega_{u 10}+U^{A} \delta \omega_{A 10}\right) \\
& =\sqrt{\bar{q}} \theta_{\text {div }}^{r}+\sqrt{\bar{q}} \theta_{\text {fin }}^{r}+o(1),
\end{aligned}
$$

where the most divergent term is a total derivative plus a total variation,

$$
-\sqrt{\bar{q}} \theta_{\operatorname{div}}^{r}=2 r \delta(\sqrt{\bar{q}} \bar{F})-\frac{r}{4} \partial_{u}\left(\sqrt{\bar{q}} C^{A B} \delta \bar{q}_{A B}\right) .
$$

\footnotetext{
${ }^{26}$ Note the possible source of confusion: $\theta^{r}$ is the "temporal" component of the flux because it is multiplied by $\xi^{u}$. Similarly for the "radial" component $\theta^{u}$.
} 
This means that it can be renormalized away by a choice of boundary Lagrangian, as shown below in section 9 . The finite contribution to the temporal flux is given by

$$
\begin{aligned}
\sqrt{\bar{q}} \theta_{\text {fin }}^{r}= & \delta\left[\sqrt{\bar{q}}\left(M+D_{C} \bar{U}^{C}\right)\right]+\frac{1}{4} \sqrt{\bar{q}} N^{A B} \delta C_{A B}+\frac{1}{2} \sqrt{\bar{q}}\left(\bar{F} C^{A B}+D^{\langle A} \bar{U}^{B\rangle}\right) \delta \bar{q}_{A B} \\
& +\left(M-\frac{1}{2} D_{C} \bar{U}^{C}-\frac{1}{4} N_{C D} C^{C D}\right) \delta \sqrt{\bar{q}}+o(1) .
\end{aligned}
$$

While for the radial flux, we have

$$
-e^{2 \beta} r^{2} \theta^{u}=r E_{i}^{A} \delta \omega_{A 1}^{i}-r^{2} \delta \omega_{r 10} .
$$

In the case of the radial flux, it is important to appreciate that $r \partial_{r}$ is an $\mathcal{O}(1)$-vector, and that all the vector fields that we are considering contain a term $\rho r \partial_{r}$ with $\rho$ finite at $\mathcal{I}$. This means that the divergent term of the radial flux that must be isolated and renormalized is $r \theta^{u}$. In light of these considerations, using the asymptotic expansion of the radial flux (see eq. (B.19) for its derivation), we can write

$$
\sqrt{\bar{q}} e^{2 \beta} r^{2} \theta^{u}=\sqrt{\bar{q}} \theta_{\mathrm{div}}^{u}+\sqrt{\bar{q}} \theta_{\mathrm{fin}}^{u}+o\left(r^{-1}\right),
$$

where the divergent component is a total derivative

$$
\sqrt{\bar{q}} \theta_{\mathrm{div}}^{u}=\frac{1}{2} \partial_{r}\left(r^{2} \delta \sqrt{\bar{q}}\right)-\frac{1}{4} \partial_{r}\left(r \sqrt{\bar{q}} C^{A B} \delta \bar{q}_{A B}\right),
$$

and the finite contribution is given by

$$
\begin{aligned}
\sqrt{\bar{q}} \theta_{\text {fin }}^{u} & =\frac{1}{4 r} C_{C D} C^{C D} \delta \sqrt{\bar{q}}-\frac{1}{4 r} \sqrt{\bar{q}} C^{A B} \delta C_{A B}-\frac{4}{r} \sqrt{\bar{q}} \delta \bar{\beta} \\
& =-\frac{4}{r} \sqrt{\bar{q}} \delta \mathrm{E}_{\beta}-\frac{1}{4 r} \sqrt{\bar{q}} C^{\langle A}{ }_{C} C^{B\rangle C} \delta \bar{q}_{A B} \hat{=} 0,
\end{aligned}
$$

where in the last equality we used the identity (B.13). The on-shell vanishing of $\theta_{\text {fin }}^{u}$ is necessary to insure that the renormalized symplectic potential $\int_{\Sigma_{u}} \theta_{\text {fin }}^{u} \epsilon_{u}$, associated with the null slice $\Sigma_{u}=\{u=$ cste $\}$, is finite.

The second contribution originating from the field dependence of the vector field, namely

$$
\mathcal{F}_{\delta \xi}:=\int_{S} q_{\delta \xi}
$$

though it is nonvanishing, its field space contraction will not contribute to the flux-balance laws that we will derive in section 8 and it does not contribute any divergent term in the expression for the fluxes.

Finally, the contribution to the Noetherian flux coming from the field-dependent gauge parameter $\lambda_{\xi}$, denoted as

$$
\mathcal{F}_{\xi}^{\lambda}:=\int_{S} q_{\delta \lambda_{\xi}}=r^{2} \int_{S} \sqrt{\bar{q}} \delta \lambda_{\xi}^{01}
$$

will give nonzero contribution to the flux-balance laws and its expression will be provided for each BMSW generator. 
In the following subsections, we provide the asymptotic expressions of the Noetherian fluxes associated to each BMSW generator in (6.2a), (6.2b), (6.2c). To lighten the notation, the three contributions to the Noetherian fluxes will be labelled by the symmetry parameter rather than its bmsw generator. For example, in the case of super-translation, we denote $\mathcal{F}_{T}^{\theta}:=\mathcal{F}_{\xi_{T}}^{\theta}$ and $\mathcal{F}_{\delta \xi_{T}}:=\mathcal{F}_{\delta T}$.

\subsection{Energy Noetherian flux}

Let us consider the super-translation generator $\xi_{T}$. The energy Noetherian flux reads as

$$
\mathcal{F}_{T}=\mathcal{F}_{T}^{\theta}+\mathcal{F}_{T}^{\lambda}+\mathcal{F}_{\delta T}
$$

The first contribution, the simplectic flux (7.2) for $\xi=\xi_{T}$, displays $r$-divergent terms coming from (7.5) and (7.9), and $\mathcal{O}(1)$ terms from (7.6) and (7.10). Collecting these contributions, one obtains that

$$
\begin{aligned}
\mathcal{F}_{T}^{\theta}= & r \int_{S}\left[2 T \delta(\sqrt{\bar{q}} \bar{F})+\frac{1}{2} \bar{\Delta} T \delta \sqrt{\bar{q}}-\frac{1}{4} \sqrt{\bar{q}} T\left(N^{A B} \delta \bar{q}_{A B}\right)\right] \\
& -\int_{S} T \delta\left[\sqrt{\bar{q}}\left(M+\bar{D}_{C} \bar{U}^{C}\right)\right]-\frac{1}{4} \int_{S} \sqrt{\bar{q}} T\left(N^{A B} \delta C_{A B}\right) \\
& -\int_{S}\left[T\left(M-\frac{1}{2} \bar{D}_{A} \bar{U}^{A}-\frac{1}{4} N_{C D} C^{C D}\right)-\frac{1}{2} \bar{U}^{A} \partial_{A} T+\frac{1}{4} \bar{D}_{A}\left(C^{A B} \partial_{B} T\right)\right] \delta \sqrt{\bar{q}} \\
& -\frac{1}{2} \int_{S} \sqrt{\bar{q}}\left(\frac{1}{4} \bar{\Delta} T C^{A B}+T \bar{F} C^{A B}+T D^{\langle A} \bar{U}^{B\rangle}\right) \delta \bar{q}_{A B}+o(1) .
\end{aligned}
$$

The second contribution to the energy Noetherian flux is

$$
\mathcal{F}_{\delta T}=\int_{S} \sqrt{\bar{q}} \delta \bar{q}^{A B} \partial_{B} T \bar{U}_{A}+o(1)
$$

The third and final contribution, namely that one coming from the field-dependent gauge parameter $\lambda$ in (7.12), for $\lambda_{\xi}^{01}=\lambda_{\xi_{T}}^{01}$ (see (5.15c) and (A.7)), reads as

$$
\mathcal{F}_{T}^{\lambda}=\frac{1}{2} \int_{S} \sqrt{\bar{q}}\left\{\frac{1}{2} \delta\left[\bar{D}_{A}\left(C^{A B} \partial_{B} T\right)\right]-\delta\left(\bar{U}^{A} \partial_{A} T\right)\right\}+o(1) .
$$

We emphasize that the divergent part of the energy flux comes uniquely from the symplectic term in the first line of (7.14). For later convenience, let us highlight it and denote it as

$$
\mathcal{F}_{T}^{\text {div }}=r \int_{S}\left[2 T \delta(\sqrt{\bar{q}} \bar{F})+\frac{1}{2} \bar{\Delta} T \delta \sqrt{\bar{q}}-\frac{1}{4} \sqrt{\bar{q}} T\left(N^{A B} \delta \bar{q}_{A B}\right)\right] .
$$

\subsection{Weyl Noetherian flux}

Let us consider the Weyl rescaling generator $\xi_{W}$. The Weyl Noetherian flux is given by

$$
\mathcal{F}_{W}=\mathcal{F}_{W}^{\theta}+\mathcal{F}_{W}^{\lambda}+\mathcal{F}_{\delta W}
$$


We follow the same computational steps as in the previous case of the energy Noetherian flux. For the Weyl Noetherian flux, considering $\xi=\xi_{W}$, its symplectic contribution can be written as

$$
\begin{aligned}
\mathcal{F}_{W}^{\theta}= & u \mathcal{F}_{T=W}^{\theta}-r \int_{S} \sqrt{\bar{q}} W \theta^{u} \\
= & u \mathcal{F}_{T=W}^{\theta}-r^{2} \int_{S} W \delta \sqrt{\bar{q}}+\frac{r}{4} \int_{S} \sqrt{\bar{q}} W C^{A B} \delta \bar{q}_{A B} \\
& -\frac{1}{4} \int_{S} W\left(C_{A B} C^{A B} \delta \sqrt{\bar{q}}-\sqrt{\bar{q}} C^{A B} \delta C_{A B}-16 \sqrt{\bar{q}} \delta \bar{\beta}\right)+o(1) .
\end{aligned}
$$

The second contribution is $\mathcal{F}_{\delta W}=u \mathcal{F}_{\delta T}$, with $T=W$ in (7.15). Finally, the contribution originating from the field-dependent gauge parameter $\lambda$ in (7.12), for $\lambda_{\xi}^{01}=\lambda_{\xi_{W}}^{01}$ (see (5.15c) and (A.8)) takes the form

$$
\mathcal{F}_{W}^{\lambda}=u \mathcal{F}_{T=W}^{\lambda}=\frac{u}{2} \int_{S} \sqrt{\bar{q}}\left\{\frac{1}{2} \delta\left[\bar{D}_{A}\left(C^{A B} \partial_{B} W\right)\right]-\delta\left(\bar{U}^{A} \partial_{A} W\right)\right\}+o(1) .
$$

Also in the Weyl case, the radial divergences come from the Weyl symplectic flux, that in turn come from the divergences in the energy symplectic flux. They are given by the following expression

$$
\begin{aligned}
\mathcal{F}_{W}^{\mathrm{div}}= & u \mathcal{F}_{T=W}^{\mathrm{div}}-r \int_{S} \sqrt{\bar{q}} W \theta_{\mathrm{div}}^{u} \\
= & 2 u r \int_{S}\left[W \delta(\sqrt{\bar{q}} \bar{F})+\frac{1}{4} \bar{\Delta} W \delta \sqrt{\bar{q}}-\frac{1}{8} \sqrt{\bar{q}} W\left(N^{A B} \delta \bar{q}_{A B}\right)\right] \\
& -r^{2} \int_{S} W \delta \sqrt{\bar{q}}+\frac{r}{4} \int_{S} \sqrt{\bar{q}} W C^{A B} \delta \bar{q}_{A B} .
\end{aligned}
$$

\subsection{Momentum Noetherian flux}

Let us consider the $\operatorname{diff}(S)$ generator $\xi_{Y}$. The momentum Noetherian flux is

$$
\mathcal{F}_{Y}=\mathcal{F}_{Y}^{\theta}+\mathcal{F}_{Y}^{\lambda}+\mathcal{F}_{\delta Y}
$$

and it is immediate to see that

$$
\mathcal{F}_{Y}=0
$$

as the sphere diffeomorphism generator $\xi_{Y}$ has only the tangential component $Y^{A} \partial_{A}$.

It is illustrative to complete the comparison with the generalized bms algebra. Let us write the corresponding Noether fluxes in terms of the BMSW ones derived above. By means of the relation (6.6) between generalized BMS and BMSW vectors fields, it is immediate to see that

$$
\mathcal{F}_{T}^{\text {gbms }}=\mathcal{F}_{T}, \quad \mathcal{F}_{Y}^{\text {gbms }}=\mathcal{F}_{W=\frac{1}{2}} \bar{D}_{A} Y^{A}
$$

This highlights how the momentum Noether charge (6.5) is Hamiltonian in the BMSW group, while it is not for the generalized BMS group of [18]. This striking feature is a reflection of the fact that, in the Bondi frame, Weyl super-boost transformations are equivalent to conformal rescalings and the Lie algebra basis $(\tau, Y)$ allows us to filter this 
component of the $\operatorname{diff}(S)$ action out of the momentum charge. On the other hand, the condition $W^{\text {gbms }}=\frac{1}{2} \bar{D}_{A} Y^{A}$ imposed in [18] to keep the scale factor constant (see section 4.3) mixes the Weyl super-boost and the sphere diffeomorphism fields, so that the resulting momentum charge is now a combination of the two and it has non-vanishing flux. In the BMS case, one recovers an Hamiltonian charge only when restricting to area-preserving diffeomorphisms $\bar{D}_{A} Y^{A}=0$, namely to the rotational part of the charge.

\section{Einstein's equations from flux-balance laws}

We are now ready to prove the main result of the paper, namely the remarkable fact that the asymptotic Einstein's equations can be obtained from the flux-balance law (2.20), where the bracket on the r.h.s. is defined by (2.14) in terms of the Noether charges (6.1) and fluxes (7.1). Before doing so, we recall the explicit expressions of the asymptotic Einstein's equations to make their connection with the flux-balance laws manifest.

\subsection{Einstein's equations in Bondi gauge}

It follows from considering the vector field $l=\partial_{r}$ and the one-form $\underline{n}=\mathrm{d} r$ (see also section 3 and figure 1), that the Einstein's equations can be grouped in four sets of equations

$$
\begin{aligned}
G_{\mu \underline{n}}=G_{\mu}{ }^{r} & =0 & \text { (constraints equations) } \\
G_{\mu l}=G_{\mu r}=-e^{2 \beta} G_{\mu}{ }^{u} & =0 & \text { (radial evolution equations) } \\
G_{\langle A B\rangle} & =0 & \text { (propagating equations) } \\
q^{C D} G_{C D} & =0 & \text { ("trivial" equation) }
\end{aligned}
$$

Among the constraints equations, we notably have in $G_{u}{ }^{r}=0$ and $G_{A}{ }^{r}=0$, respectively, the (retarded time) evolution for the Bondi mass and the Bondi angular momentum aspects.

We list the Einstein tensor with mixed indices $G_{\mu}{ }^{\nu}$ obtained in the Bondi gauge. In the following, $\mathrm{E}_{\Phi}$ stands for the asymptotic Einstein's equations for the fields $\left\{\bar{F}, \bar{\beta}, \bar{U}, M, \bar{P}_{A}\right\}$ defined in (2.27), (2.28) and (2.29).

The four radial evolution equations $G_{\mu}{ }^{u}=0$ give

$$
\begin{aligned}
G_{u}{ }^{u} & =-\frac{1}{2 r^{2}} \mathrm{E}_{\bar{F}}-\frac{1}{r^{3}} \bar{D}^{A} \mathrm{E}_{\bar{U}_{A}}+o\left(r^{-3}\right), \\
G_{r}{ }^{u} & =\frac{8}{r^{4}} \mathrm{E}_{\bar{\beta}}+o\left(r^{-4}\right), \\
G_{A}{ }^{u} & =\frac{1}{r^{2}} \mathrm{E}_{\bar{U}_{A}}+o\left(r^{-2}\right) .
\end{aligned}
$$

In addition, we have the constraints equations $G_{\mu}{ }^{r}=0$, with

$$
\begin{aligned}
G_{u}{ }^{r} & =\frac{2}{r^{2}}\left(\mathrm{E}_{M}+\frac{1}{2} \bar{D}^{A} \dot{\mathrm{E}}_{\bar{U}_{A}}\right)+o\left(r^{-2}\right), \\
G_{r}{ }^{r} & =-\frac{1}{2 r^{2}} \mathrm{E}_{\bar{F}}-\frac{1}{r^{3}} \bar{D}^{A} \mathrm{E}_{\bar{U}_{A}}+o\left(r^{-3}\right), \\
G_{A}{ }^{r} & =-\frac{1}{r} \dot{\mathrm{E}}_{\bar{U}_{A}}+\frac{1}{r^{2}}\left(\mathrm{E}_{\bar{P}_{A}}+2 \bar{D}_{A} \dot{\mathrm{E}}_{\bar{\beta}}-2 \bar{F} \mathrm{E}_{\bar{U}_{A}}-\frac{1}{2} \bar{U}_{A} \mathrm{E}_{\bar{F}}\right)+o\left(r^{-2}\right) .
\end{aligned}
$$


The remaining Einstein's equations are

$$
\begin{aligned}
G_{\langle A B\rangle} & =-\frac{1}{2 r} \mathrm{E}_{\bar{F}} C_{A B}+\frac{1}{r^{2}}\left(\dot{E}_{A B}+\cdots\right)+o\left(r^{-2}\right), \\
g^{C D} G_{C D} & =-\frac{1}{r^{2}} \mathrm{E}_{\bar{F}}-\frac{2}{r^{3}}\left(\bar{D}^{A} \mathrm{E}_{\bar{U}_{A}}-8 \dot{\mathrm{E}}_{\bar{\beta}}\right)+o\left(r^{-3}\right) .
\end{aligned}
$$

We also recall that $G_{\langle A B\rangle}=\mathcal{R}_{\langle A B\rangle}$, where the sphere components of the $4 \mathrm{~d}$ Ricci tensor and the $4 \mathrm{~d}$ Ricci scalar are given respectively by

$$
\begin{aligned}
\mathcal{R}_{A B} & =\frac{1}{2} \mathrm{E}_{\bar{F}} \bar{q}_{A B}+\frac{1}{r} \bar{D}^{C} \mathrm{E}_{\bar{U}_{C}} \bar{q}_{A B}+o\left(r^{-1}\right), \\
\mathcal{R} & =2 g^{u r} \mathcal{R}_{u r}+g^{r r} \mathcal{R}_{r r}+2 g^{r A} \mathcal{R}_{r A}+g^{A B} \mathcal{R}_{A B} \\
& =\frac{1}{r^{2}} \mathrm{E}_{\bar{F}}+\frac{1}{r^{3}}\left(2 \bar{D}^{A} \mathrm{E}_{\bar{U}_{A}}-8 \dot{\mathrm{E}}_{\bar{\beta}}\right)+o\left(r^{-3}\right),
\end{aligned}
$$

Two comments are in order here. First, the propagating equations, $G_{\langle A B\rangle}=0$, contain the evolution equation for the symmetric and traceless field $E_{A B}$, that appears in the asymptotic expansion of $q_{A B}$ (see section 2.2 above) and enters the Weyl scalar $\psi_{0}$ (see appendix D). The dots in the $\mathcal{O}\left(r^{-2}\right)$ coefficient stand for additional terms that are not important for the present discussion (see more on this in the Conclusions section 10). Second, in the expressions of the Einstein tensor above, we have set the boundary condition $\partial_{u} \bar{q}_{A B}=0$ in eq. (2.27) to streamline the presentation of the Einstein tensor components. However, the reader can find it useful to know that, without that condition, the leading orders of $G_{\mu}{ }^{\nu}$ change as follows: $G_{u}{ }^{u}=\mathcal{O}\left(r^{-1}\right), G_{r}{ }^{u}=\mathcal{O}\left(r^{-1}\right), G_{u}{ }^{r}=\mathcal{O}(1), G_{A}{ }^{r}=\mathcal{O}(1)$ and $G_{A B}=\mathcal{O}(r)$.

\subsection{Flux-balance laws}

We are now ready to evaluate the flux balance laws (2.15), which can be written as

$$
\delta_{\xi} Q_{\chi}-I_{\chi} \mathcal{F}_{\xi}+\int_{S} \iota_{\xi} \iota_{\chi} L+Q_{\llbracket \xi, \chi \rrbracket}=r^{2} \int_{S} \sqrt{q} e^{2 \beta}\left(\xi^{u} \chi^{\mu} G_{\mu}^{r}-\xi^{r} \chi^{\mu} G_{\mu}^{u}\right)
$$

To make the flux-balance laws manifest, we use the fact that $\chi=\hat{u}:=\partial_{u} \in$ BMSW, and its corresponding variation $\delta_{\hat{u}}$ is a time derivative, thus providing a term $\dot{Q}_{\xi}$ in (8.7). The expression of the Noether charge is given by

$$
Q_{\hat{u}}=\int_{S} \sqrt{\bar{q}} M,
$$

and the expression of the Noetherian flux reads as

$$
\begin{aligned}
\mathcal{F}_{\hat{u}}= & r \int_{S}\left(2 \delta(\sqrt{\bar{q}} \bar{F})-\frac{1}{4} \sqrt{\bar{q}} N^{A B} \delta \bar{q}_{A B}\right) \\
& -\int_{S} \delta\left(\sqrt{\bar{q}}\left(M+\bar{D}_{A} \bar{U}^{A}\right)\right)-\int_{S}\left(M-\frac{1}{2} D_{C} \bar{U}^{C}-\frac{1}{4} N_{C D} C^{C D}\right) \delta \sqrt{\bar{q}} \\
& -\frac{1}{2} \int_{S} \sqrt{\bar{q}}\left(\bar{F} C^{A B}+D^{\langle A} \bar{U}^{B\rangle}\right) \delta \bar{q}_{A B}-\frac{1}{4} \int_{S} \sqrt{q} N^{A B} \delta C_{A B}+o(1) .
\end{aligned}
$$


In our proof, we concentrate on the $(T, W, Y)$ basis $(6.2 \mathrm{a}),(6.2 \mathrm{~b}),(6.2 \mathrm{c})$ which satisfy the Lie algebra (4.22). This allows us to readily identify which residual diffeomorphism transformation relates the associated charge bracket to the (holographically) equivalent asymptotic Einstein's equations. Specifically, we will specialize the pair $(\xi, \chi)$ to $\left(\xi_{T, W, Y}, \partial_{u}\right)$ and the flipped order $\left(\partial_{u}, \xi_{T, W, Y}\right)$. The computation uses the Noether charges derived in section 6 and the Noetherian fluxes obtained in section 7 .

For later convenience, let us write the Lagrangian as

$$
L=\frac{1}{2} \epsilon \mathcal{R},
$$

and use the 4d Ricci scalar in (8.6) to compute the inner products of the Lagrangian form with time translations $\partial_{u}$ and the three bmsw generators

$$
\begin{aligned}
& \int_{S} \iota_{\xi_{T}} \iota_{\hat{u}} L=\frac{1}{4} \int_{S} \sqrt{\bar{q}} \bar{\Delta} T \mathrm{E}_{\bar{F}}+o(1), \\
& \int_{S} \iota_{\xi_{W}} \iota_{\hat{u}} L=-\frac{1}{2} \int_{S} \sqrt{\bar{q}}\left[W\left(r \mathrm{E}_{\bar{F}}+2 \bar{D}^{A} \mathrm{E}_{\bar{U}_{A}}-8 \dot{\mathrm{E}}_{\beta}\right)-\frac{u}{2} \bar{\Delta} W \mathrm{E}_{\bar{F}}\right]+o(1), \\
& \int_{S} \iota_{\xi_{Y}} \iota_{\hat{u}} L=0 .
\end{aligned}
$$

These expressions will be shortly needed to evaluate the brackets (2.14) in the flux-balance relations (2.15).

\subsubsection{Energy flux-balance}

We apply the general expression (8.7) to write the energy flux-balance law, respectively, with $(\xi, \chi)=\left(\partial_{u}, \xi_{T}\right)$ and $(\xi, \chi)=\left(\xi_{T}, \partial_{u}\right)$. They are

$$
\begin{aligned}
& \delta_{\hat{u}} Q_{T}-I_{T} \mathcal{F}_{\hat{u}}+\int_{S} \iota_{\hat{u}} \iota_{\xi} L+Q_{\llbracket \hat{u}, \xi_{T} \rrbracket}=\int_{S} \sqrt{\bar{q}} T\left(2 \mathrm{E}_{M}-\frac{1}{4} \bar{\Delta} \mathrm{E}_{\bar{F}}\right)+o(1), \\
& \delta_{T} Q_{\hat{u}}-I_{\hat{u}} \mathcal{F}_{T}+\int_{S} \iota_{\xi_{T}} \iota_{\hat{u}} L+Q_{\llbracket \xi_{T}, \hat{u} \rrbracket}=\int_{S} \sqrt{\bar{q}} T\left(2 \mathrm{E}_{M}+\bar{D}^{A} \dot{\mathrm{E}}_{\bar{U}_{A}}+\frac{1}{4} \bar{\Delta} \mathrm{E}_{\bar{F}}\right)+o(1) .
\end{aligned}
$$

We first focus on the first expression (8.12a), for which the energy charge bracket

$$
\left\{Q_{\hat{u}}, Q_{\xi_{T}}\right\}:=\delta_{\hat{u}} Q_{T}-I_{T} \mathcal{F}_{\hat{u}}+\int_{S} \iota_{\hat{u}} \iota_{\xi_{T}} L=-Q_{\llbracket \hat{u}, \xi_{T} \rrbracket}
$$

is equivalent to combination of asymptotic Einstein's equations

$$
B:=2 \mathrm{E}_{M}-\frac{1}{4} \bar{\Delta} \mathrm{E}_{\bar{F}}=0 .
$$

We provide the proof by separately computing all the terms in eq. (8.13). By means of eq. (6.3), the variation with respect to time of the energy Noether charge is given by

$$
\delta_{\hat{u}} Q_{T}=\int_{S} \sqrt{\bar{q}} T\left(\dot{M}-\frac{1}{2} \bar{D}_{A} \dot{\bar{U}}^{A}\right) .
$$


We then use the expression of the flux $\mathcal{F}_{\hat{u}}$ in (8.9) and the field variations under supertranslations in (C.1) to compute the field space contraction on $\mathcal{F}_{\hat{u}}$

$$
\begin{aligned}
I_{T} \mathcal{F}_{\hat{u}} & =-\int_{S} \sqrt{q} \delta_{T} M-\frac{1}{4} \int_{S} \sqrt{q} N^{A B} \delta_{T} C_{A B} \\
& =-\int_{S} \sqrt{q} T\left(\dot{M}-\bar{\Delta} \bar{F}-\frac{3}{4} \bar{D}_{A} \bar{D}_{B} N^{A B}+\frac{1}{4} N^{A B} N_{A B}+\frac{1}{2} \bar{D}^{A} \dot{\mathrm{E}}_{\bar{U}_{A}}\right) .
\end{aligned}
$$

Finally, by means of the commutators (4.15), we have $\llbracket \hat{u}, \xi_{T} \rrbracket=0$, and hence $Q_{\llbracket \hat{u}, \xi_{T} \rrbracket}=0$. Therefore, it is straightforward to see that the energy flux-balance law (8.13), after adding up eqs. (8.15), (8.16), and (8.11a), yields the condition (8.14).

From $(8.12 \mathrm{~b})$, we see that flipping the order of the vector fields in the energy charge bracket (8.13), which can be read off from the right-hand side of (8.12b),

$$
\delta_{T} Q_{\hat{u}}-I_{\hat{u}} \mathcal{F}_{T}+\int_{S} \iota_{\xi_{T}} \iota_{\hat{u}} L=Q_{\llbracket \hat{u}, \xi_{T} \rrbracket}
$$

is expected to be equivalent to the following linear combination of asymptotic Einstein's equations

$$
B^{\prime}:=2 \mathrm{E}_{M}+\bar{D}^{A} \dot{\mathrm{E}}_{\bar{U}_{A}}+\frac{1}{4} \bar{\Delta} \mathrm{E}_{\bar{F}}=0 .
$$

We can prove this statement again by evaluating the different terms in (8.17) separately. First, we compute the variation of $Q_{\hat{u}}$ under super-translations

$$
\delta_{T} Q_{\hat{u}}=\int_{S} \sqrt{\bar{q}} T\left(\dot{M}-\bar{\Delta} \bar{F}-\frac{1}{4} \bar{D}_{A} \bar{D}_{B} N^{A B}+\frac{1}{2} \bar{D}_{A} \dot{\mathrm{E}}_{\bar{U}_{A}}\right)
$$

then the field contraction of the energy Noetherian flux

$$
I_{\hat{u}} \mathcal{F}_{T}=-\int_{S} \sqrt{\bar{q}} T\left(\dot{M}+\bar{D}_{A} \dot{\bar{U}}^{A}+\frac{1}{4} N^{A B} N_{A B}-\frac{1}{2} \bar{D}_{A} \dot{\bar{U}}^{A}\right) .
$$

By means of (8.11a) and $Q_{\llbracket \hat{u}, \xi_{T} \rrbracket}=0$, we obtain exactly (8.18).

We will see in a the next subsection how both (8.14) and (8.18) are automatically satisfied by the asymptotic Einstein's equations implied by the Weyl flux-balance law.

\subsubsection{Weyl flux-balance}

In the case of conformal transformations, the flux-balance law $(8.7)$ with $(\xi, \chi)=\left(\partial_{u}, \xi_{W}\right)$ and $(\xi, \chi)=\left(\xi_{W}, \partial_{u}\right)$ is given, respectively, by

$$
\begin{aligned}
\delta_{\hat{u}} Q_{W} & -I_{W} \mathcal{F}_{\hat{u}}+\int_{S} \iota_{\hat{u}} \iota_{\xi_{W}} L+Q_{\llbracket \hat{u}, \xi_{W} \rrbracket} \\
& =\int_{S} \sqrt{\bar{q}} W\left[\frac{r}{2} \mathrm{E}_{\bar{F}}+\bar{D}^{A} \mathrm{E}_{\bar{U}_{A}}+2 u\left(\mathrm{E}_{M}-\frac{1}{8} \Delta \mathrm{E}_{\bar{F}}\right)\right]+o(1), \\
\delta_{W} Q_{\hat{u}} & -I_{\hat{u}} \mathcal{F}_{W}+\int_{S} \iota_{\xi_{W}} \iota_{\hat{u}} L+Q_{\llbracket \xi_{W}, \hat{u} \rrbracket} \\
& =\int_{S} \sqrt{\bar{q}} W\left[-\frac{r}{2} \mathrm{E}_{\bar{F}}-\bar{D}^{A} \mathrm{E}_{\bar{U}_{A}}+2 u\left(\mathrm{E}_{M}+\frac{1}{2} \bar{D}^{A} \dot{\mathrm{E}}_{\bar{U}_{A}}+\frac{1}{8} \Delta \mathrm{E}_{\bar{F}}\right)\right]+o(1) .
\end{aligned}
$$


We focus on the first Weyl charge bracket

$$
\delta_{\hat{u}} Q_{W}-I_{W} \mathcal{F}_{\hat{u}}+\int_{S} \iota_{\hat{u}} \iota \xi_{W} L=-Q_{\llbracket \hat{u}, \xi_{W} \rrbracket},
$$

and show that it is equivalent to the following combination of the asymptotic Einstein's equations

$$
A+u B=0,
$$

with

$$
A:=\frac{r}{2} \mathrm{E}_{\bar{F}}+\bar{D}_{A} \mathrm{E}_{\bar{U}_{A}},
$$

and $B$ given in (8.14), as expected from the r.h.s. of (8.21a).

By means of eq. (6.4), we compute the time variation of the Weyl Noether charge

$$
\delta_{\hat{u}} Q_{W}=4 \int_{S} \sqrt{\bar{q}} W \dot{\bar{\beta}}+u \int_{S} \sqrt{\bar{q}} W\left(\dot{M}-\frac{1}{2} \bar{D}_{A} \dot{\bar{U}}^{A}\right) .
$$

We then use the field variations under the Weyl rescalings in (C.2) to compute the field contraction on $\mathcal{F}_{\hat{u}}$

$$
\begin{aligned}
I_{W} \mathcal{F}_{\hat{u}}= & \int_{S} \sqrt{\bar{q}} W\left(M-\frac{1}{4} \bar{D}_{A} \bar{D}_{B} C^{A B}-\bar{D}_{A} \bar{U}^{A}-\frac{1}{4} N^{A B} C_{A B}+\frac{1}{2} \bar{D}_{A} \mathrm{E}_{\bar{U}_{A}}\right) \\
& -u \int_{S} \sqrt{\bar{q}} W\left(\dot{M}-\bar{\Delta} \bar{F}-\frac{3}{4} \bar{D}_{A} \bar{D}_{B} N^{A B}+\frac{1}{4} N^{A B} N_{A B}+\frac{1}{2} \bar{D}_{A} \dot{\mathrm{E}}_{\bar{U}_{A}}\right) .
\end{aligned}
$$

Finally, by means of the commutators $(4.15)$, we have $\llbracket \hat{u}, \xi_{W} \rrbracket=\xi_{T=W}$, so that

$$
Q_{\llbracket \hat{u}, \xi_{W} \rrbracket}=\int_{S} \sqrt{\bar{q}} W\left(M-\frac{1}{2} \bar{D}_{A} \bar{U}^{A}\right) .
$$

Therefore, upon substituting eqs. (8.25), (8.26), and (8.11b), the Weyl flux-balance law (8.22) yields exactly the condition (8.32).

On the other hand, to expand the flipped version of (8.22), namely

$$
\delta_{W} Q_{\hat{u}}-I_{\hat{u}} \mathcal{F}_{W}+\int_{S} \iota_{\xi_{W}} \iota \hat{u} L=-Q_{\llbracket \xi_{W}, \hat{u} \rrbracket},
$$

we first compute the variation under Weyl transformations of the $Q_{\hat{u}}$ and obtain

$$
\begin{aligned}
\delta_{W} Q_{\hat{u}}= & \int_{S} \sqrt{\bar{q}} W\left(M+\frac{1}{4} \bar{D}_{A} \bar{D}_{B} C^{A B}-\frac{1}{2} \bar{D}_{A} \mathrm{E}_{\bar{U}_{A}}\right) \\
& +u \int_{S} \sqrt{\bar{q}} W\left(\dot{M}-\bar{\Delta} \bar{F}-\frac{1}{4} \bar{D}_{A} \bar{D}_{B} N^{A B}+\frac{1}{2} \bar{D}_{A} \dot{\mathrm{E}}_{\bar{U}_{A}}\right),
\end{aligned}
$$

and the field contraction of the Weyl Noetherian flux

$$
\begin{aligned}
I_{\hat{u}} \mathcal{F}_{W}= & \frac{1}{4} \int_{S} \sqrt{\bar{q}} W C^{A B} N_{A B}+4 \int_{S} \sqrt{\bar{q}} W \dot{\bar{\beta}} \\
& -u \int_{S} \sqrt{\bar{q}} W\left(\dot{M}+\frac{1}{2} \bar{D}_{A} \dot{\bar{U}}^{A}+\frac{1}{4} N^{A B} N_{A B}\right) .
\end{aligned}
$$


Therefore, the flipped Weyl flux-balance law (8.33) yields a condition of the form $A^{\prime}+u B^{\prime}=$ 0 , where

$$
A^{\prime}:=-\frac{r}{2} \mathrm{E}_{\bar{F}}-\bar{D}_{A} \mathrm{E}_{\bar{U}_{A}},
$$

and $B^{\prime}$ given in (8.18), consistently with the r.h.s. of (8.21b).

We thus conclude that the flux-balance laws (8.21) for Weyl rescaling transformations yield two asymptotic Einstein's equations in eq. (2.27), namely $A=-A^{\prime}=0$ implies

$$
\bar{D}_{A} \bar{U}^{A}=-\frac{1}{2} \bar{D}_{A} \bar{D}_{B} C^{A B} \text {. }
$$

and

$$
\bar{F}=\frac{\bar{R}(\bar{q})}{4} .
$$

By plugging (8.32), (8.33) into the condition $B=0$ (or equivalently $B^{\prime}=0$ ), we recover the evolution equation for the Bondi mass $[18,21]$

$$
\dot{M}=\frac{1}{4} \bar{D}_{A} \bar{D}_{B} \dot{C}^{A B}-\frac{1}{8} N^{A B} N_{A B}+\frac{1}{8} \bar{\Delta} \bar{R} .
$$

These equations immediately satisfy the conditions obtained in eqs. (8.14), (8.18) from the energy balance law. Therefore, the asymptotic Einstein's equations $\mathrm{E}_{\bar{F}}=0, \mathrm{E}_{M}=0, \mathrm{E}_{\bar{U}_{A}}=$ 0 are indeed recovered from the energy and the Weyl flux-balance laws.

\subsubsection{Momentum flux-balance}

In the case of sphere diffeomorphisms, the momentum flux-balance law with $(\xi, \chi)=$ $\left(\partial_{u}, \xi_{Y}\right)$ reads $\operatorname{as}^{27}$

$$
\begin{aligned}
\delta_{\hat{u}} Q_{Y} & -I_{Y} \mathcal{F}_{\hat{u}}+\int_{S} \iota_{\hat{u}} \iota_{\xi_{Y}} L+Q_{\llbracket \hat{u}, \xi_{Y} \rrbracket} \\
& =\int_{S} \sqrt{\bar{q}} Y^{A}\left[-r \dot{\mathrm{E}}_{\bar{U}_{A}}+\mathrm{E}_{\bar{P}_{A}}+2 \partial_{A} \dot{\mathrm{E}}_{\bar{\beta}}-2 \bar{F} \mathrm{E}_{\bar{U}_{A}}-\frac{1}{2} \bar{U}_{A} \mathrm{E}_{\bar{F}}\right] .
\end{aligned}
$$

Be aware that the divergent contribution in $G_{A}{ }^{r}$, namely $-r \dot{\mathrm{E}}_{\bar{U}_{A}}$ in the right-hand side of the above equation, is exactly cancelled out by the divergent contributions appearing in $\delta_{\hat{u}} Q_{Y}$ and $I_{Y} \mathcal{F}_{\hat{u}}$ given below. Consistently, in the renormalization procedure carried out in section 9 , it is shown that the Einstein's equation $\mathrm{E}_{\bar{U}_{A}}=0$ needs to be imposed in order to obtain a finite expression for the momentum charge.

With these considerations in mind, we see from (8.35) that on-shell of the asymptotic Einstein's for $\bar{U}^{A}, \bar{F}$ and $M$ just derived, the momentum charge bracket

$$
\delta_{\hat{u}} Q_{Y}-I_{Y} \mathcal{F}_{\hat{u}}+\int_{S} \iota_{\hat{u}} \iota_{\xi_{Y}} L=-Q_{\llbracket \hat{u}, \xi_{Y} \rrbracket}
$$

is expected to be equivalent to the extra Einstein's equations

$$
\mathrm{E}_{\bar{P}_{A}}+2 \partial_{A} \dot{\mathrm{E}}_{\bar{\beta}}=0
$$

as suggested by the r.h.s. of (8.35).

\footnotetext{
${ }^{27}$ The flipped version is trivial; in fact, $\delta_{Y} Q_{\hat{u}}=0, \mathcal{F}_{Y}=0$ and the other terms in the flux-balance give vanishing contribution.
} 
We verify this statement proceeding as above. By means of eq. (6.5), we compute the time variation of the momentum charges

$$
\delta_{\hat{u}} Q_{Y}=-r \int_{S} \sqrt{\bar{q}} Y^{A} \dot{\bar{U}}_{A}+\int_{S} \sqrt{\bar{q}} Y^{A}\left(\dot{\bar{P}}_{A}+2 \partial_{A} \dot{\bar{\beta}}\right) .
$$

We then make use of the field variations under the vector $Y^{A}$ in (C.3) which in particular imply that

$$
\int_{S} \delta_{Y}(\sqrt{\bar{q}} \bar{F})=0, \quad \int_{S} \delta_{Y}(\sqrt{\bar{q}} M)=0, \quad \int_{S} \delta_{Y}\left(\sqrt{\bar{q}} \bar{D}_{A} \bar{U}^{A}\right)=0
$$

to compute the field contraction on $\mathcal{F}_{\hat{u}}$

$$
\begin{aligned}
I_{Y} \mathcal{F}_{\hat{u}}= & \frac{r}{2} \int_{S} \sqrt{\bar{q}} Y_{A} \bar{D}_{B} N^{A B} \\
& +\int_{S} \sqrt{\bar{q}} Y^{A} \partial_{A} M+\int_{S} \sqrt{\bar{q}} Y^{A} C_{A B} \bar{D}^{B} \bar{F} \\
& +\frac{1}{4} \int_{S} \sqrt{\bar{q}} Y^{C}\left[\bar{D}_{B}\left(N^{A B} C_{C A}-C^{A B} N_{C A}\right)-N^{A B} \bar{D}_{C} C_{A B}\right] \\
& +\frac{1}{2} \int_{S} \sqrt{\bar{q}} Y^{A}\left(\bar{D}_{B} \bar{D}^{B} \bar{U}_{A}-\bar{D}_{B} \bar{D}_{A} \bar{U}^{B}\right)+o(1) .
\end{aligned}
$$

In the derivation of the expression above, we have used

$$
\bar{D}_{B} \bar{D}_{A} \bar{U}^{B}=\bar{D}_{A} \bar{D}_{B} \bar{U}^{B}+2 \bar{F} \bar{U}_{A}
$$

which follows from the commutator $\left[\bar{D}_{A}, \bar{D}_{C}\right]$ applied to the vector $\bar{U}^{A}$, and the relation (B.15). Finally, by means of the commutators (4.15), we have $\llbracket \hat{u}, \xi_{Y} \rrbracket=0$, implying that $Q_{\llbracket \hat{u}, \xi_{Y} \rrbracket}=0$.

Therefore, the momentum charge bracket (8.36), on-shell of the asymptotic equations (8.32), which removes the divergent contributions, and (8.33), yields

$$
\begin{aligned}
\dot{\bar{P}}_{A}+2 \partial_{A} \dot{\bar{\beta}}= & \partial_{A} M+C_{A B} \bar{D}^{B} \bar{F} \\
& +\frac{1}{4} \bar{D}_{B}\left(N^{A B} C_{A C}-C^{A B} N_{A C}\right)-\frac{1}{4} N^{B C} \bar{D}_{A} C_{B C} \\
& +\frac{1}{4}\left(\bar{D}_{B} D_{A} \bar{D}_{C} C^{B C}-\bar{D}_{B} D^{B} \bar{D}^{C} C_{A C}\right)
\end{aligned}
$$

which can be rewritten in the form (8.37) as expected. We thus recover the momentum evolution equation $(2.29)$ (see also $[18,21]$ ) in linear combination with the asymptotic Einstein's equation for $\bar{\beta}$ in $(2.27 \mathrm{~b})$. It is straightforward to verify that the reversed version of the momentum balance law (8.36) trivially gives $0=0$. The reader might also appreciate the fact that the combination $\dot{\bar{P}}_{A}+2 \partial_{A} \dot{\bar{\beta}}$ is indeed the (time derivative of the) renormalized BMSW momentum aspect (2.43). Its derivation is provided in the next section.

This completes the derivation of the asymptotic Einstein's equations at null infinity from the flux-balance laws defined by the bracket (2.20) for an open Hamiltonian system. 


\section{Charge and flux renormalization}

We now want to investigate the renormalization procedure for the charges and for the symplectic flux outlined in section 2.3. The limit to infinity is taken by first considering the spacetime boundary $\mathcal{B}=\Sigma_{-} \cup \Delta \cup \Sigma_{+}$, with $\Sigma_{ \pm}, \Delta$ codimension-1 hypersurfaces respectively given by $u=u_{ \pm}, r=r_{\Delta}$, with $u_{+}>u_{-}$and then taking the $\operatorname{limit}^{28} r_{\Delta} \rightarrow+\infty$. The different components of the symplectic potential are given by

$$
\Theta_{ \pm}=\int_{\Sigma_{ \pm}} \theta=\int_{\Sigma_{ \pm}} r^{2} \sqrt{q} e^{2 \beta} \theta^{u} \mathrm{~d} r \mathrm{~d}^{2} \sigma, \quad \Theta_{\Delta}=-\int_{\Delta} \theta=r^{2} \int_{\Delta} \sqrt{q} e^{2 \beta} \theta^{r} \mathrm{~d} u \mathrm{~d}^{2} \sigma
$$

$\Theta_{ \pm}$represents the symplectic potential on different time slices, while $\Theta_{\Delta}$ represents the symplectic potential flux leaking through the boundary. The fact that the Lagrangian variation vanishes on-shell implies the conservation equation

$$
\Theta_{+} \hat{=} \Theta_{-}+\Theta_{\Delta}
$$

We can use the results (7.5), (7.9) to write the divergent part of $\Theta_{\mathcal{B}}$ as

$$
\theta_{\text {div }}=\mathrm{d} \vartheta_{\operatorname{div}}-\frac{r}{2} \delta\left(\sqrt{\bar{q}} \mathrm{E}_{\bar{F}}\right) \mathrm{d} u \mathrm{~d}^{2} \sigma,
$$

where the corner symplectic potential is

$$
\vartheta_{\text {div }}=\left(\frac{r^{2}}{2} \delta(\sqrt{\bar{q}})-\frac{r}{4} \sqrt{\bar{q}} C^{A B} \delta \bar{q}_{A B}\right) \mathrm{d}^{2} \sigma+r \vartheta^{A} \epsilon_{A B} \mathrm{~d} \sigma^{B} \wedge \mathrm{d} u,
$$

and we have introduced the vector valued variational form

$$
\vartheta_{A}:=\frac{1}{2} \sqrt{q} \bar{D}^{B}\left(\delta \bar{q}_{A B}-q_{A B} q^{C D} \delta q_{C D}\right) .
$$

This form appears in the variation of the scalar curvature

$$
\frac{1}{2} \delta(\sqrt{\bar{q}} \bar{R}(\bar{q}))=\partial_{A} \vartheta^{A} .
$$

Therefore, by means of the Einstein's equation $\mathrm{E}_{\bar{F}}=0,{ }^{29}$ we can define the renormalized symplectic potential in the form (2.30) with $\ell_{\text {div }}=0$ on-shell of the boundary condition $\partial_{u} \bar{q}_{A B}=0$, namely

$$
\theta^{R}=\theta-\mathrm{d} \vartheta_{\mathrm{div}} .
$$

This yields the renormalized symplectic 2-form (2.36). According to (2.31) and (2.32), the renormalized charge and flux are given by

$$
\begin{aligned}
& Q_{\xi}^{R}=Q_{\xi}-\int_{S} I_{\xi} \vartheta_{\mathrm{div}}, \\
& \mathcal{F}_{\xi}^{R}=\mathcal{F}_{\xi}-\int_{S} \delta_{\xi} \vartheta_{\mathrm{div}} .
\end{aligned}
$$

\footnotetext{
${ }^{28}$ The limit $u_{ \pm} \rightarrow \pm \infty$ will be considered elsewhere.

${ }^{29}$ Imposition of this equation is not necessary for the renormalization of the potential, but for the energy and Weyl charges and fluxes, as otherwise, according to (2.30), an extra divergent term would appear in the renormalized expressions (2.31), (2.32).
} 


\subsection{Renormalized charges}

We can compute the renormalized charge aspects given by (9.8). In the case of the energy aspect, we use (C.1) and (6.3) to compute

$$
Q_{T}^{R}=Q_{T}-\int_{S} I_{\xi_{T}} \vartheta_{\mathrm{div}}=\int_{S} \sqrt{\bar{q}} T\left(M-\frac{1}{2} \bar{D}_{A} \bar{U}^{A}\right)
$$

In the case of the Weyl aspect, we use (C.2) and (6.4) to compute

$$
Q_{W}^{R}=Q_{W}-\int_{S} I_{\xi_{W}} \vartheta_{\mathrm{div}}=\int_{S} \sqrt{\bar{q}} W\left[4 \bar{\beta}+u\left(M-\frac{1}{2} \bar{D}_{A} \bar{U}^{A}\right)\right] .
$$

Notice that the internal Lorentz contribution to the charge (5.7), namely $\int_{\Sigma} I_{\lambda} \theta$, does not contribute to the charge renormalization term as $\int_{S} I_{\lambda} \vartheta_{\text {div }}=0$. It is also interesting to appreciate that the divergence of the Weyl aspect (6.4) is entirely due to the Lorentz charge contribution (A.8). The renormalization term $\int_{S} I_{\xi_{W}} \vartheta_{\text {div }}$ for the Weyl aspect cancels exactly this internal Lorentz divergence. This highlights again the importance of taking into account also the internal $\mathrm{SL}(2, \mathbb{C})$ contribution to the charges to get finite expressions.

In the case of the momentum aspect, we use (C.3), (6.5) and the Einstein's equation $\mathrm{E}_{\bar{U}_{A}}=0$ to compute

$$
Q_{Y}^{R}=Q_{Y}-\int_{S} I_{\xi_{Y}} \vartheta_{\mathrm{div}}=\int_{S} \sqrt{\bar{q}} Y^{A}\left(\bar{P}_{A}+2 \partial_{A} \bar{\beta}\right)
$$

We thus see how the renormalization procedure removes exactly the radially divergent terms in the Noether charge, but leaves the finite terms unmodified. The renormalized BMS charges $Q_{(T, Y)}^{R-\text { gbms }}=Q_{\left(T, W=\frac{1}{2} \bar{D}_{A} Y^{A}, Y\right)}^{R}$ are given by

$$
Q_{(T, Y)}^{R-\text { gbms }}=\int_{S} \sqrt{\bar{q}}\left[\tau\left(M-\frac{1}{2} \bar{D}_{A} \bar{U}^{A}\right)+Y^{A} \bar{P}_{A}\right],
$$

where $\tau=T+\frac{u}{2} \bar{D}_{A} Y^{A}$.

\subsection{Relation to Barnich-Troessaert charges}

One puzzle we face is that the Noetherian charges that we constructed from the covariant Lagrangian are not the same as the one considered by Barnich-Troessaert [21] or FlanaganNichols [17] and studied further by Compere et al. [18, 51]. Given the emphasis we have put on the Noetherian split's relevance, one has to wonder whether these previous charges are obtainable from a choice of boundary Lagrangian. We now show that this is indeed the case: the BT charges are recovered from our covariant Noether charges after adding a non-covariant boundary Lagrangian.

To that end, let us consider a family of boundary Lagrangians, parametrized by one parameter $\alpha \in \mathbb{R}$,

$$
\ell_{\alpha}:=\sqrt{\bar{q}}\left(M+\frac{\alpha}{8} C^{A B} N_{A B}\right) \mathrm{d} u \mathrm{~d}^{2} \sigma .
$$

This gives the corner symplectic potential

$$
\vartheta_{\alpha}=\frac{\alpha}{8} \sqrt{\bar{q}}\left(C^{A B} \delta C_{A B}\right) \mathrm{d}^{2} \sigma .
$$


Applying the general formula (2.16) and thanks to the identity (B.13), the shift in the charge $Q_{(\xi ; \alpha)}^{R}-Q_{\xi}^{R}$ due to the presence of the boundary Lagrangian is thus given by

$$
\int_{S}\left(i_{\xi} \ell_{\alpha}-I_{\xi} \vartheta_{\alpha}\right)=\int_{S} \sqrt{\bar{q}}\left[\tau M+\frac{\alpha}{8}\left(\dot{\tau}-\frac{1}{2} \bar{D}_{C} Y^{C}\right) C^{A B} C_{A B}+\frac{\alpha}{4} C^{A B} \bar{D}_{A} \partial_{B} \tau\right] .
$$

Applying this to the super translation charge and integrating by part gives

$$
Q_{(T ; \alpha)}^{R}=\int_{S} \sqrt{\bar{q}} T\left(2 M-\frac{1}{2}(1+\alpha) \bar{D}_{A} \bar{U}^{A}\right) .
$$

For the Weyl charge one obtains

$$
Q_{(W ; \alpha)}^{R}=\int_{S} \sqrt{\bar{q}} W\left[4(1-\alpha) \bar{\beta}+u\left(2 M-\frac{1}{2}(1+\alpha) \bar{D}_{A} \bar{U}^{A}\right)\right],
$$

where we have used the asymptotic EEs for $\bar{U}^{A}$ and $\bar{\beta}$. A similar calculation shows that the shifted expression of the renormalized sphere diffeomorphism charge is

$$
Q_{(Y ; \alpha)}^{R}=\int_{S} \sqrt{\bar{q}} Y^{A}\left(\bar{P}_{A}+2(1-\alpha) \partial_{A} \bar{\beta}\right)
$$

The full Noether charge can then be written as

$$
\begin{aligned}
Q_{(T, W, Y ; \alpha)}^{R}=\int_{S} \sqrt{\bar{q}}[(T+u W) & \left(2 M-\frac{1}{2}(1+\alpha) \bar{D}_{A} \bar{U}^{A}\right) \\
& \left.+2(1-\alpha)\left(2 W-\bar{D}_{A} Y^{A}\right) \bar{\beta}+Y^{A} \bar{P}_{A}\right] .
\end{aligned}
$$

We can thus see that the charge considered by Barnich-Troessart [21] or FlanaganNichols [17] is recovered for $\alpha=-1$ once we set $W=\frac{1}{2} \bar{D}_{A} Y^{A}$, namely

$$
Q_{(T, Y)}^{\mathrm{BT}}=Q_{\left(T, W=\frac{1}{2} \bar{D}_{A} Y^{A}, Y ;-1\right)}^{R}=\int_{S} \sqrt{\bar{q}}\left[2 \tau M+Y^{A} \bar{P}_{A}\right],
$$

where $\tau=T+\frac{u}{2} \bar{D}_{A} Y^{A}$.

As pointed out in section 2.1 (and derived in [44]), the non-covariance of the boundary Lagrangian (9.14) introduces an extra contribution, $K_{(\xi, \chi)}$, in the bracket (2.18) in terms of the boundary Lagrangian anomaly. This extra contribution is given in (2.19) and it can be computed using the anomalous transformation terms (that can be read off from (4.41))

$$
\begin{aligned}
\Delta_{\tau} C_{A B} & =-2 \bar{D}_{\langle A} \partial_{B\rangle} \tau \\
\Delta_{\tau} N_{A B} & =-2 \bar{D}_{\langle A} \partial_{B\rangle} \dot{\tau} \\
\Delta_{\tau} M & =\left(\frac{1}{2} \bar{D}_{A} N^{A B}+\partial^{B} \bar{F}\right) \partial_{B} \tau+\frac{1}{4} N^{A B} \bar{D}_{A} \partial_{B} \tau+\frac{1}{4} C^{A B} \bar{D}_{A} \partial_{B} \dot{\tau} .
\end{aligned}
$$

This yields

$$
\begin{aligned}
\Delta_{\tau} \ell_{-1} & =\sqrt{\bar{q}}\left(\Delta_{\tau} M-\frac{1}{8}\left(\Delta_{\tau} C^{A B} N_{A B}+C^{A B} \Delta_{\tau} N_{A B}\right)\right) \mathrm{d} u \wedge \mathrm{d}^{2} \sigma \\
& =\sqrt{\bar{q}}\left[\partial^{A} \bar{F} \partial_{A} \tau+\frac{1}{2} \bar{D}_{A}\left(N^{A B} \partial_{B} \tau\right)+\frac{1}{2} C^{A B} \bar{D}_{A} \partial_{B} \dot{\tau}\right] \mathrm{d} u \wedge \mathrm{d}^{2} \sigma,
\end{aligned}
$$


from which (we use the notation $\iota_{\tau}:=\iota_{\xi_{\tau}}$ )

$$
\begin{aligned}
K_{\left(\tau_{1}, \tau_{2}\right)} & =\int_{S}\left(\iota_{\tau_{1}} \Delta_{\tau_{2}} \ell_{-1}-\iota_{\tau_{2}} \Delta_{\tau_{1}} \ell_{-1}\right) \\
& =\frac{1}{4} \int_{S} \sqrt{\bar{q}}\left[\tau_{1} \partial^{A} \bar{R} \partial_{A} \tau_{2}+\tau_{1} C^{A B} \bar{D}_{A} \partial_{B} \bar{D}_{C} Y_{2}^{C}-1 \leftrightarrow 2\right] .
\end{aligned}
$$

This expression for the cocycle, first proposed in [47], matches exactly the one for the 2-cocycle in the Barnich-Troessaert bracket for the charges (9.21) derived in [51].

This establishes cleanly that the BT charge is obtained from the addition of the Lagrangian $\ell_{-1}$. It is important to note however that $\ell_{-1}$ is not integrable on $\mathcal{I}$ and therefore this polarization does not seem to be accessible if we want agreement between Hamiltonian and Lagrangian formulations. The non-integrability of $\ell_{-1}$ comes from the fact that when $u \rightarrow \infty$ we have that $\mathcal{M} \rightarrow 0$, which means that

$$
\ell_{\alpha} \rightarrow \frac{1}{8}(\alpha-1) N_{\mathrm{vac}}^{A B} C_{A B}^{\mathrm{vac}}
$$

The only value for which this converges to 0 is $\alpha=1$. This means that the BT split of charge and flux cannot accommodate the presence of a non-trivial asymptotic Weyl frame.

\subsection{Renormalized Noetherian fluxes}

We can finally compute the renormalized Noetherian fluxes given by (9.9). In the case of the energy Noetherian flux, by means of the (6.2a), we have

$$
\begin{aligned}
\mathcal{F}_{T}^{R} & =\mathcal{F}_{T}-\int_{S} \delta_{T} \vartheta_{\mathrm{div}} \\
& =\mathcal{F}_{T}+\frac{r}{4} \int_{S} \sqrt{\bar{q}} T N^{A B} \delta \bar{q}_{A B}+\frac{r}{2} \int_{S} \sqrt{\bar{q}} T \bar{D}_{A} \bar{D}_{B} \delta \bar{q}^{A B}+\frac{r}{2} \int_{S} \bar{\Delta} T \delta \sqrt{\bar{q}} \\
& =\mathcal{F}_{T}+\frac{r}{4} \int_{S} \sqrt{\bar{q}} T N^{A B} \delta \bar{q}_{A B}-2 r \int_{S} T \delta(\sqrt{\bar{q}} \bar{F})-\frac{r}{2} \int_{S} \bar{\Delta} T \delta \sqrt{\bar{q}}
\end{aligned}
$$

where we have used (8.33), (B.12a), (B.12b), and the relation

$$
\frac{1}{2} \sqrt{\bar{q}} \bar{D}_{A} \bar{D}_{B} \delta \bar{q}^{A B}=-\frac{1}{2} \delta(\sqrt{\bar{q}} \bar{R}(\bar{q}))-\bar{\Delta} \delta \sqrt{\bar{q}}
$$

which follows from (9.6).

In the case of the Weyl symplectic flux, by means of the (6.2b), similar manipulations for the energy symplectic flux show that we have

$$
\begin{aligned}
\mathcal{F}_{W}^{R}= & \mathcal{F}_{W}-\int_{S} \delta_{W} \vartheta_{\mathrm{div}} \\
= & \mathcal{F}_{W}-\frac{r}{4} \int_{S} C_{A B} \delta \bar{q}^{A B} \delta_{W} \sqrt{\bar{q}}-\frac{r}{4} \int_{S} \sqrt{\bar{q}} \delta_{W} C_{A B} \delta \bar{q}^{A B} \\
& -\frac{r}{4} \int_{S} \sqrt{\bar{q}} C_{A B} \delta \delta_{W} \bar{q}^{A B}-\frac{r^{2}}{2} \int_{S} \delta\left(\delta_{W} \sqrt{\bar{q}}\right) \\
= & \mathcal{F}_{W}-\frac{r}{4} \int_{S} \sqrt{\bar{q}} W C^{A B} \delta \bar{q}_{A B}+r^{2} \int_{S} W \delta \sqrt{\bar{q}} \\
& +\frac{r u}{4} \int_{S} \sqrt{\bar{q}} W N^{A B} \delta \bar{q}_{A B}-2 r u \int_{S} W \delta(\sqrt{\bar{q}} \bar{F})-\frac{r u}{2} \int_{S} \bar{\Delta} W \delta \sqrt{\bar{q}}
\end{aligned}
$$


In the case of the momentum symplectic flux, by means of the (6.2c), it is straightforward to see that

$$
\mathcal{F}_{Y}^{R}=\mathcal{F}_{Y}-\int_{S} \delta_{Y} \vartheta_{\text {div }},=0
$$

where we use the fact that $\mathcal{F}_{Y}=0$ and that $\delta_{Y}$ has no anomaly and therefore it can be replaced by $\mathcal{L}_{Y}$.

We thus see that the renormalization procedure for the charges $Q_{\xi}$ and fluxes $\mathcal{F}_{\xi}$ computed above removes the divergent terms in the Noetherian expressions obtained from (6.1) and (7.1) and does not pick up any new finite term (see the expressions (7.17), (7.21) for the divergent parts of the fluxes). Similarly for $Q_{\hat{u}}^{R}=Q_{\hat{u}}$ and

$$
\mathcal{F}_{\hat{u}}^{R}=\mathcal{F}_{\hat{u}}-\int_{S} \delta_{\hat{u}} \vartheta_{\operatorname{div}}=\mathcal{F}_{\hat{u}}+\frac{r}{4} \int_{S} \sqrt{\bar{q}} N^{A B} \delta \bar{q}_{A B} .
$$

It follows immediately that the derivation of the asymptotic Einstein's equations presented in section 8 goes through in exactly the same way if working at null infinity to begin with and using the renormalized Noetherian quantities for the bracket (2.14). This is no surprise of course, as it represents an explicit check of the invariance property of the bracket (2.20) under the shift (2.16). Finally we can also evaluate the shifted renormalized flux associated with the boundary Lagrangian (9.14). This can be easily done following the procedure outlined so far.

\section{Conclusions}

In this work, we have achieved five interconnected results:

1. We have shown that, under the boundary conditions proposed by $[16,18]$, the asymptotic symmetry group of flat space null infinity is given by the BMSW group, which contains super-translations, Diff(S) transformations, and Weyl super-boosts. We also have shown that the BMS group is obtained as field-dependent reduction of BMSW.

2. Following [44], we have given a generalization of the Barnich-Troessaert bracket and shown that demanding that this bracket provides a representation of the symmetry algebra implies 5 asymptotic Einstein's equations: $\mathrm{E}_{\bar{F}}$ and $D_{A} \mathrm{E}_{\bar{U}^{A}}$ as well as the energy and momenta conservation equation $\mathrm{E}_{M}$ and $\mathrm{E}_{\bar{P}_{A}}+2 \dot{\mathrm{E}}_{\bar{\beta}}$. These results are summarized in table 1 .

3. We have provided the holographic renormalization of the symplectic potential and the BMSW charges. We have shown that the renormalized symplectic potential is finite provided the asymptotic equation $\mathrm{E}_{\bar{\beta}}$ is satisfied. The renormalized charges are also finite provided $\mathrm{E}_{\bar{F}}, \mathrm{E}_{\bar{U}^{A}}$ are satisfied. This result extends the analysis performed by Compere et al. in [18] by including Weyl transformations and the $2 \mathrm{~d}$ metric determinant as a phase space variable.

4. We have shown that we can obtain the Barnich-Troessaert [21] BMS charges from a Noetherian split associated with a non-covariant Lagrangian defined in (9.14). 
5. Finally, we have shown that the vacuum structure of asymptotically flat gravity is labeled by a BMSW group element. This contains a super-translation and a Weyl label similar to the one revealed by Compère and Long [69]. In addition it contains a $\operatorname{Diff}(S)$ label.

These results emphasize the importance of choosing a Noetherian split associated with a choice of Lagrangian. They also generalize to asymptotic infinity the results of [44] obtained at a finite distance for the extended corner symmetry group. In particular, the remarkable connection, through the flux laws (2.15), between Einstein's equations and the canonical representation of the symmetry algebra (be it finite or asymptotic) could be understood as the core element of (local) holography. This suggests a deep connection between finite and asymptotic symmetry algebra (see also [32] for such a connection).

One could extend our results in several ways: first, one could try to relax the condition $\partial_{u} \bar{q}_{A B}=0$ and authorize a time-dependent background metric. Essential elements of this generalizations have been worked out in [21], but in a restricted context that does not allow the full Diff(S) symmetry.

Also, our analysis reveals that the bigger the symmetry group, the more Einstein's equations can be recast as identities of canonical brackets or flux-balance laws. It thus suggests that the maximal symmetry group should be defined as the one that recovers all Einstein's equations, not a subset of them. Our study is still missing the two purely space-like Einstein's equations ${ }^{30} G_{\langle A B\rangle}=0$. This means that we expect the full symmetry group to be bigger than BMSW. We conjecture that the full extension includes dual supertranslations associated to the dual energy charges [110]. One way to reveal these symmetry transformations is to introduce the Immirzi parameter by considering the Einstein-CartanHolst action and repeating the analysis performed here for the dual component of its symplectic potential (5.2). This will be addressed in a forthcoming work.

One issue that needs to be understood better is the nature of the limit $u \rightarrow \pm \infty$ of the Noether charges. In this limit one pushes the sphere $S$ to spacelike and timelike infinities, which are fixed by the symmetry transformations and therefore we do not expect the presence of non-trivial fluxes. The charges that survive this limit are the covariant mass and the covariant momentum which are related to the Weyl tensor. This corresponds to the proposal of [51]. It is not clear however if these covariant charges can be obtained as limits of the Noetherian ones.

At the same time, it would be interesting to understand the connection between the BMSW symmetry algebra and the soft theorems more deeply. Especially, what is the role of the newly revealed Weyl charge in this context, and whether the new $\operatorname{Diff}(S)$ vacua label could be related to a new memory effect associated with the spin memory effect as a vacuum transition $[19,123]$.

And finally, now that we have a bona fide Diff(S) symmetry group and not just a super-Lorentz symmetry acting on the gravitational phase space, one can wonder whether this means that there exists a fluid description of the asymptotic conservation equations that extends what is found at the corner [41].

\footnotetext{
${ }^{30}$ Recall that we obtained five EEs from the charge bracket and two more from the renormalization procedure, while $G_{A}^{A}=0$ follows trivially from these.
} 


\section{Acknowledgments}

We would like to thank Glenn Barnich, Geoffrey Compère, Adrien Fiorucci, Romain Ruzziconi, Anthony Speranza for helpful discussions and insights. R. O. thanks Geoffrey Compère, Ali Seraj, Bernard Whiting for collaborations on related topics over the past years, and is grateful to Constantinos Skordis for his support and advice. We thank A. M. for lifting our spirits. R. O. is funded by the European Structural and Investment Funds (ESIF) and the Czech Ministry of Education, Youth and Sports (MSMT), Project CoGraDSCZ.02.1.01/0.0/ 0.0/15003/0000437. Research at Perimeter Institute is supported in part by the Government of Canada through the Department of Innovation, Science and Economic Development Canada and by the Province of Ontario through the Ministry of Colleges and Universities. This project has received funding from the European Union's Horizon 2020 research and innovation programme under the Marie Sklodowska-Curie grant agreement No. 841923.

\section{A Asymptotic expansions}

In this appendix we collect all asymptotic expansions used to derive the results in the main text.

\section{Metric coefficients:}

$$
\begin{aligned}
q^{A B} & =\bar{q}^{A B}-\frac{1}{r} C^{A B}+\frac{1}{4 r^{2}} \bar{q}^{A B} C_{C D} C^{C D}+\mathcal{O}\left(r^{-2}\right) \\
D_{A} V^{A} & =\frac{1}{\sqrt{q}} \partial_{A}\left(\sqrt{q} V^{A}\right)=\frac{1}{\sqrt{\bar{q}}} \partial_{A}\left(\sqrt{\bar{q}} V^{A}\right)=\bar{D}_{A} V^{A} \\
I^{A B} & =\int_{r}^{\infty} \frac{\mathrm{d} r^{\prime}}{r^{\prime 2}} e^{2 \beta} q^{A B}=\frac{1}{r} \bar{q}^{A B}-\frac{1}{2 r^{2}} C^{A B}+\frac{1}{16 r^{2}} \bar{q}^{A B} C^{C D} C_{C D}+o\left(r^{-3}\right) .
\end{aligned}
$$

Tetrad coefficients:

$$
\begin{aligned}
& E_{A}^{i}=\bar{E}_{A}^{i}+\frac{1}{2 r} C_{A}{ }^{B} \bar{E}_{B}^{i}+\frac{1}{16 r^{2}} \bar{E}_{A}^{i} C_{B C} C^{B C}+o\left(r^{-2}\right), \\
& \hat{E}_{i}^{A}=\bar{E}_{i}^{A}-\frac{1}{2 r} C^{A}{ }_{B} \bar{E}_{i}^{B}+\frac{1}{16 r^{2}} \bar{E}_{i}^{A} C_{B C} C^{B C}+o\left(r^{-2}\right) .
\end{aligned}
$$

\section{Spin connection:}

$$
\omega_{10}=\left[\frac{M}{r^{2}}+\mathcal{O}\left(r^{-2}\right)\right] \mathrm{d} u+\left[-\frac{4 \bar{\beta}}{r^{3}}+o\left(r^{-3}\right)\right] \mathrm{d} r+\left[-\frac{\bar{U}_{A}}{r}+\frac{1}{r^{2}}\left(\bar{P}_{A}+2 \bar{D}_{A} \bar{\beta}\right)\right] \mathrm{d} x^{A} .
$$

\section{Gauge parameters:}

$$
\begin{aligned}
\lambda_{T}^{01} & =\frac{1}{2 r^{2}}\left[\frac{1}{2} \bar{D}_{A}\left(C^{A B} \partial_{B} T\right)-\bar{U}^{A} \partial_{A} T\right]+o\left(r^{-2}\right), \\
\lambda_{W}^{01} & =-W+\frac{u}{2 r^{2}}\left[\frac{1}{2} \bar{D}_{A}\left(C^{A B} \partial_{B} W\right)-\bar{U}^{A} \partial_{A} W\right]+o\left(r^{-2}\right), \\
\lambda_{Y}^{01} & =0 .
\end{aligned}
$$




\section{B Derivation the symplectic flux}

In this appendix, we compute the symplectic flux $\mathcal{F}_{\xi}^{\theta}:=\int_{S} \iota_{\xi} \theta$. We first expand the contraction of the EC symplectic potential (5.2) with a generic vector field $\xi$. We use the convention that $\epsilon_{01 i j}=\epsilon_{i j}$ and the notation $\xi^{a} e_{a}^{I}=\xi^{I}$. We have

$$
\begin{aligned}
\mathcal{F}_{\xi}^{\theta} & =\frac{1}{4} \epsilon_{I J K L} \int_{S}\left(2 i_{\xi} e^{K} e^{L} \wedge \delta \omega^{I J}+e^{K} \wedge e^{L} \iota_{\xi} \delta \omega^{I J}\right) \\
& =\frac{1}{2} \int_{S}\left[r \epsilon_{I J K \ell} \xi^{K} E_{A}^{\ell}\left(\delta \omega_{B}^{I J}\right)+r^{2} \epsilon_{i j} E_{A}^{i} E_{B}^{j}\left(\iota_{\xi} \delta \omega^{01}\right)\right] \epsilon^{A B} \mathrm{~d}^{2} \sigma \\
& =r \int_{S} \epsilon_{i j} E_{A}^{j} \epsilon^{A B}\left[\xi^{i}\left(\delta \omega_{B}^{01}\right)-\xi^{1}\left(\delta \omega_{B}^{0 i}\right)+\xi^{0}\left(\delta \omega_{B}{ }^{1 i}\right)\right] \mathrm{d}^{2} \sigma+r^{2} \int_{S_{\infty}} \sqrt{q}\left(\iota_{\xi} \delta \omega^{01}\right) \mathrm{d}^{2} \sigma \\
& =r \int_{S} \sqrt{q}\left[E^{i A}\left(\xi^{0} \delta \omega_{A 0 i}-\xi^{1} \delta \omega_{A 1 i}-\xi_{i} \delta \omega_{A 10}\right)+r \iota_{\xi} \delta \omega_{10}\right] \mathrm{d}^{2} \sigma
\end{aligned}
$$

In the last equality we have used that $\epsilon_{C A} \epsilon^{A B}=-\sqrt{q} \delta_{C}^{B}$ and $\epsilon_{i j} \epsilon^{A B} E_{A}^{i}=\sqrt{q} E_{j}^{B}$.

Next, we use the identities

$$
\xi^{i} E_{i}^{A}=r\left(\xi^{A}-U^{A} \xi^{u}\right), \quad \xi^{0}=e^{2 \beta} \xi^{u}, \quad \xi^{1}=\xi^{r}+F \xi^{u},
$$

to evaluate the symplectic flux as

$$
\begin{aligned}
\mathcal{F}_{\xi}^{\theta}= & r \int_{S} \sqrt{q} \xi^{u}\left[E_{i}^{A}\left(e^{2 \beta} \delta \omega_{A 0}{ }^{i}-F \delta \omega_{A 1}{ }^{i}\right)+r U^{A} \delta \omega_{A 10}\right] \\
& -r \int_{S} \sqrt{q} \xi^{r}\left(E_{i}^{A} \delta \omega_{A 1}{ }^{i}\right)+r^{2} \int_{S} \sqrt{q}\left(\iota_{\xi} \delta \omega_{10}-\xi^{A} \delta \omega_{A 10}\right) .
\end{aligned}
$$

This can be rewritten as in eq. (7.2), namely

$$
\begin{aligned}
\mathcal{F}_{\xi}^{\theta}= & \int_{S} \sqrt{q}\left(\xi^{r} \theta^{u}-\xi^{u} \theta^{r}\right) \\
= & r \int_{S} \sqrt{q} \xi^{u}\left[E_{i}^{A}\left(e^{2 \beta} \delta \omega_{A 0}^{i}-F \delta \omega_{A 1}^{i}\right)+r\left(\delta \omega_{u 10}+U^{A} \delta \omega_{A 10}\right)\right] \\
& -r \int_{S} \sqrt{q} \xi^{r}\left(E_{i}^{A} \delta \omega_{A 1}{ }^{i}-r \delta \omega_{r 10}\right) .
\end{aligned}
$$

\section{B.1 Asymptotic expansion of the temporal flux}

We want to expand the temporal flux

$$
-\theta^{r}=r\left[E_{i}^{A}\left(e^{2 \beta} \delta \omega_{A 0 i}-F \delta \omega_{A 1 i}\right)+r\left(\delta \omega_{u 10}+U^{A} \delta \omega_{A 10}\right)\right] .
$$

First, we focus on the last two terms

$$
\begin{aligned}
r^{2}\left(\delta \omega_{u 10}+U^{A} \delta \omega_{A 10}\right) & =r^{2} \delta\left(\omega_{u 10}+U^{A} \omega_{A 10}\right)-r^{2} \delta U^{A} \omega_{A 10} \\
& =\delta\left(r^{2} F^{\prime}+2 r^{2} \beta^{\prime} F\right)-r \delta U^{A} P_{A} \\
& =\delta M+o(1)
\end{aligned}
$$

where we have used eq. (A.6) and the asymptotic expansions (2.26). 
Now, we expand two terms of (B.5). They give

$$
\begin{aligned}
e^{2 \beta} \delta \omega_{B 0 i}-F \delta \omega_{B 1 i} & =e^{2 \beta} \delta\left[e^{-2 \beta} E_{i}^{A}\left(F S_{A B}-K_{A B}\right)\right]+F \delta\left(E_{i}^{A} S_{A B}\right) \\
& =\delta F E_{i}^{A} S_{A B}-\delta\left(E_{i}^{A} K_{A B}\right)+2 F \delta\left(E_{i}^{A} S_{A B}\right)-2 \delta \beta E_{i}^{A}\left(F S_{A B}-K_{A B}\right) .
\end{aligned}
$$

We can contract this with $E^{B i}$, using that $\delta E_{i}^{(A} E^{B) i}=\frac{1}{2} \delta q^{A B}$ and denoting $S=q^{A B} S_{A B}$ and $K=q^{A B} K_{A B}$. In particular, we use the following relation

$$
E^{B i} \delta\left[E_{i}^{A} K_{A B}\right]=\frac{1}{2} K_{A B} \delta q^{A B}+q^{A B} \delta K_{A B}=\delta K-\frac{1}{2} K_{A B} \delta q^{A B}
$$

to get

$$
\begin{aligned}
r\left(e^{2 \beta} \delta \omega_{B 0 i}-F \delta \omega_{B 1 i}\right) E^{B i}= & r\left[S \delta F+\frac{1}{2} K_{A B} \delta q^{A B}-\delta K+2 F \delta S-F S_{A B} \delta q^{A B}\right] \\
& -2 r \delta \beta(F S-K) .
\end{aligned}
$$

Therefore, we have

$$
\begin{aligned}
-\theta^{r} & =r\left[E_{i}^{A}\left(e^{2 \beta} \delta \omega_{A 0}{ }^{i}-F \delta \omega_{A 1}{ }^{i}\right)+r\left(\delta \omega_{u 10}+U^{A} \delta \omega_{A 10}\right)\right] \\
& =[\delta M+o(1)]+r\left[\delta(S F-K)+\frac{1}{2} K_{A B} \delta q^{A B}+F \delta S-F S_{A B} \delta q^{A B}\right] .
\end{aligned}
$$

To perform the asymptotic expansion, we need the following expressions

$$
\begin{aligned}
q^{A B} & =\bar{q}^{A B}-\frac{1}{r} C^{A B}+o\left(r^{-1}\right), \\
r K_{A B} & =\left(\frac{1}{2} D_{C} \bar{U}^{C}+\frac{1}{4} N_{C D} C^{C D}\right) \bar{q}_{A B}+\frac{r}{2} N_{\langle A B\rangle}+D_{\langle A} \bar{U}_{B\rangle}+o(1), \\
r K & =D_{C} \bar{U}^{C}+o(1), \\
S_{A B} & =\bar{q}_{A B}+\frac{C_{A B}}{2 r}+o\left(r^{-1}\right), \\
S & =2+o\left(r^{-1}\right),
\end{aligned}
$$

where $\langle A B\rangle$ denote the symmetric traceless component. By adding (B.6) and (B.8), we get the asymptotic expression of the temporal flux

$$
\begin{aligned}
-\theta^{r}= & r\left(2 \delta \bar{F}-\bar{F} \bar{q}_{A B} \delta \bar{q}^{A B}+\frac{1}{4} N_{A B} \delta \bar{q}^{A B}\right)-\frac{1}{4} N_{A B} \delta C^{A B}+\frac{1}{2}\left(\bar{D}_{\langle A} \bar{U}_{B\rangle}+\bar{F} C_{A B}\right) \delta \bar{q}^{A B} \\
& -\left(\frac{1}{2} D_{C} \bar{U}^{C}+\frac{1}{4} N_{C D} C^{C D}+2 M\right) \delta \ln \sqrt{q}-\delta\left(M+D_{C} \bar{U}^{C}\right)+o(1),
\end{aligned}
$$

where we used $\bar{F} \bar{q}_{A B} \delta \bar{C}^{A B}=\bar{F} \delta \bar{q}^{A B} \bar{C}_{A B}$ and $\delta \ln \sqrt{q}=-\frac{1}{2} \bar{q}_{A B} \delta \bar{q}^{A B}$.

To further simplify the above expression, we use the following variations

$$
\begin{aligned}
\delta \bar{q}^{A B} & =-\bar{q}^{A C} \bar{q}^{B D} \delta \bar{q}_{C D}, \\
\delta C^{A B} & =\delta\left(\bar{q}^{A C} \bar{q}^{B D} C_{C D}\right)=C_{C}{ }^{B} \delta \bar{q}^{A C}+C^{A}{ }_{C} \delta \bar{q}^{B C}+\bar{q}^{A C} \bar{q}^{B D} \delta C_{C D},
\end{aligned}
$$


and the properties that a symmetric and traceless $2 \times 2$ matrix $C_{A B}$ obey

$$
\begin{aligned}
C_{A C} C^{C}{ }_{B} & =\frac{1}{2} \bar{q}_{A B} C_{C D} C^{C D} \\
N_{A C} N^{C}{ }_{B} & =\frac{1}{2} \bar{q}_{A B} N_{C D} N^{C D} \\
N_{A C} C^{C}{ }_{B} & =-C_{A C} N^{C}{ }_{B}+\bar{q}_{A B} N_{C D} C^{C D} \\
\dot{N}_{A C} C^{C}{ }_{B} & =-C_{A C} \dot{N}_{B}{ }_{B}+\bar{q}_{A B} \dot{N}_{C D} C^{C D} .
\end{aligned}
$$

Note, in particular, that eq. (B.15) implies

$$
N^{\langle A} C^{B\rangle C} \delta \bar{q}_{A B}=0
$$

This gives us the final expression for the time flux

$$
\begin{aligned}
-\sqrt{\bar{q}} \theta^{r}= & 2 \delta(r \sqrt{\bar{q}} \bar{F})-\frac{1}{4} \partial_{u}\left(r \sqrt{\bar{q}} C^{A B} \delta \bar{q}_{A B}\right) \\
& -\delta\left[\sqrt{\bar{q}}\left(M+D_{C} \bar{U}^{C}\right)\right]-\frac{1}{4} \sqrt{\bar{q}} N^{A B} \delta C_{A B}-\frac{1}{2} \sqrt{\bar{q}}\left(\bar{F} C^{A B}+D^{\langle A} \bar{U}^{B\rangle}\right) \delta \bar{q}_{A B} \\
& -\left(M-\frac{1}{2} D_{C} \bar{U}^{C}-\frac{1}{4} N_{C D} C^{C D}\right) \delta \sqrt{\bar{q}}+o(1)
\end{aligned}
$$

where we have used the asymptotic condition that $\partial_{u} \bar{q}_{A B}=0$ and that $N_{A B}=\partial_{u} C_{A B}$ to rewrite the divergent contributions in the first line as a total time derivative.

\section{B.2 Asymptotic expansion of the radial flux}

Likewise it was done for the temporal flux, we can show that the radial flux (7.7) is given by

$$
\begin{aligned}
\sqrt{\bar{q}} \theta^{u}= & -r \sqrt{\bar{q}}\left(E^{A i} \delta\left(\omega_{1 i}\right)_{A}-r \delta\left(\omega_{10}\right)_{r}\right) \\
= & r \sqrt{\bar{q}} E^{A i} \delta\left(E_{i}^{B} S_{B A}\right)+2 r^{2} \sqrt{\bar{q}} \delta \beta^{\prime} \\
= & r \sqrt{\bar{q}}\left(\delta S-\frac{1}{2} S_{A B} \delta q^{A B}\right)-\frac{4}{r} \sqrt{\bar{q}} \delta \bar{\beta} \\
= & \frac{r}{2} \sqrt{\bar{q}} \bar{q}{ }^{A B} \delta q_{A B}+\frac{1}{2} \sqrt{\bar{q}} \bar{q} A B \delta C^{A B}-\frac{1}{4} \sqrt{\bar{q}} C_{A B} \delta \bar{q}^{A B} \\
& -\frac{1}{2 r} \sqrt{\bar{q}} \bar{q}_{A B} \delta\left(C^{A} C_{C} C^{C B}-\frac{1}{4} \bar{q}^{A B} C_{C D} C^{C D}\right) \\
& +\frac{1}{4 r} \sqrt{\bar{q}} C_{A B} \delta C^{A B}-\frac{4}{r} \sqrt{\bar{q}} \delta \bar{\beta}+o\left(r^{-1}\right) \\
= & r \delta \sqrt{\bar{q}}-\frac{1}{4} \sqrt{\bar{q}} C^{A B} \delta \bar{q} \\
& +\frac{1}{4 r} C_{C D} C^{C D} \delta \sqrt{\bar{q}}-\frac{1}{4 r} \sqrt{\bar{q}} C^{A B} \delta C_{A B}-\frac{4}{r} \sqrt{\bar{q}} \delta \bar{\beta}+o\left(r^{-1}\right),
\end{aligned}
$$

where we used again the properties (B.12), (B.13), and the fact that $\sqrt{\bar{q}} \bar{q}_{A B} \delta \bar{q}^{A B}=-2 \delta \sqrt{\bar{q}}$. 


\section{Variations of fields}

In this appendix, we list the field variations under the bmsw generators of those fields that enter the Noether charges and fluxes. This list of variations is derived from the field variations in section 4.4 and it is useful to perform the computation outlined in section 8 when performing the field variations of the Noether charges and the field contractions on the Noether charges.

Field variations under super-translation, written off-shell of the $\mathrm{E}_{\bar{U}_{A}}$ equation of motion:

$$
\begin{aligned}
\delta_{T} \bar{q}_{A B} & =0 \\
\delta_{T} \bar{F} & =0 \\
\delta_{T} M & =T \dot{M}+\partial^{A} T \partial_{A} \bar{F}+\frac{1}{4} N^{A B} \bar{D}_{A} \bar{D}_{B} T+\frac{1}{2} \partial_{A} T\left(\bar{D}_{B} N^{A B}-\dot{\mathrm{E}}_{\bar{U}_{A}}\right), \\
\delta_{T} C_{A B} & =T N_{A B}-2 \bar{D}_{A} \bar{D}_{B} T+\bar{q}_{A B} \bar{\Delta} T \\
\delta_{T}\left(\bar{D}_{A} \bar{U}^{A}\right) & =\bar{D}_{A} \delta_{T} \bar{U}^{A} .
\end{aligned}
$$

Field variations under the Weyl rescaling, also off-shell of $\mathrm{E}_{\bar{U}_{A}}=0$ :

$$
\begin{aligned}
\delta_{W} \bar{q}_{A B}= & -2 W \bar{q}_{A B}, \\
\delta_{W} \sqrt{\bar{q}}= & -2 W \sqrt{\bar{q}} \\
\delta_{W} \bar{F}= & 2 W \bar{F}+\frac{1}{2} \bar{\Delta} W \\
\delta_{W} M= & W \dot{M}+3 W M+u \partial^{A} W \partial_{A} \bar{F} \\
& +\frac{1}{4} C^{A B} \bar{D}_{A} \partial_{B} W+\frac{u}{2}\left(\bar{D}_{A} N^{A B}-\dot{\mathrm{E}}_{\bar{U}_{B}}\right) \partial_{B} W \\
& +\frac{u}{4} N^{A B} \bar{D}_{A} \bar{D}_{B} W+\frac{1}{2} \mathrm{E}_{\bar{U}_{A}} \partial_{A} W \\
\delta_{W} C_{A B}= & -W C_{A B}+u W N_{A B}+u \bar{\Delta} W \bar{q}_{A B}-2 u \bar{D}_{A} \bar{D}_{B} W, \\
\delta_{W} N_{A B}= & u W \dot{N}_{A B}+\bar{\Delta} W \bar{q}_{A B}-2 \bar{D}_{A} \bar{D}_{B} W \\
\delta_{W}\left(\bar{D}_{A} \bar{U}^{A}\right)= & \bar{D}_{A}\left(\delta_{W} \bar{U}^{A}\right)-2 \bar{U}^{A} \partial_{A} W .
\end{aligned}
$$

Field variations under the sphere diffeomorphism $Y^{A}$

$$
\begin{aligned}
\delta_{Y} \bar{q}_{A B} & =2 \bar{D}_{(A} Y_{B)}, \\
\delta_{Y} \sqrt{\bar{q}} & =\bar{D}_{A} Y^{A} \sqrt{\bar{q}}, \\
\delta_{Y} \bar{F} & =Y^{A} \bar{D}_{A} \bar{F},
\end{aligned}
$$




$$
\begin{aligned}
\delta_{Y} M & =Y^{A} \bar{D}_{A} M \\
\delta_{Y} C_{A B} & =Y^{C} \bar{D}_{C} C_{A B}+2 C_{C(A} \bar{D}_{B)} Y^{C} \\
\delta_{Y}\left(\bar{D}_{A} \bar{U}^{A}\right) & =-\bar{D}_{A} Y^{A} \bar{D}_{C} \bar{U}^{C}+\frac{1}{\sqrt{q}} \partial_{C}\left(\delta_{Y}\left(\sqrt{\bar{q}} \bar{U}^{C}\right)\right) .
\end{aligned}
$$

\section{Weyl scalars in Bondi gauge}

To compute the Weyl scalars, we recall the frame field in eq. (5.9b)

$$
\hat{e}_{0}=e^{-2 \beta}\left(\partial_{u}-F \partial_{r}+U^{A} \partial_{A}\right), \quad \hat{e}_{1}=\partial_{r}, \quad \hat{e}_{2}=\frac{1}{r} \hat{E}_{2}^{A} \partial_{A}, \quad \hat{e}_{3}=\frac{1}{r} \hat{E}_{3}^{A} \partial_{A} .
$$

We also recall that $q^{A B}=\hat{E}_{i}^{A} \hat{E}_{j}^{B} \eta^{i j}=2 \hat{E}_{2}^{(A} \hat{E}_{3}^{B)}$ and $\epsilon^{A B}=\hat{E}_{i}^{A} \hat{E}_{j}^{B} \epsilon^{i j}=2 \hat{E}_{2}^{[A} \hat{E}_{3}^{B]}$.

The five Weyl scalars are defined by contracting the Weyl tensor $W_{\mu \nu \rho \sigma}$ with the frame field above. It is more useful, in order to highlight the covariant feature of these quantities, to introduce the following definitions

$$
\begin{aligned}
\left(\Psi_{0}\right)_{A B} & =-3\left(E_{A B}-\frac{1}{16} C_{A B} C_{C D} C^{C D}\right), & \left(\Psi_{4}\right)_{A B} & =-\frac{1}{2} \dot{N}_{A B}, \\
\left(\Psi_{1}\right)_{A} & =-\mathcal{P}_{A}, & \left(\Psi_{3}\right)_{A} & =-\frac{1}{2}\left(\frac{1}{2} \bar{D}_{A} \bar{R}+\bar{D}_{B} N_{A}^{B}\right), \\
\operatorname{Re} \Psi_{2} & =-\left(M+\frac{1}{8} C^{A B} N_{A B}\right), & \operatorname{Im} \Psi_{2} & =-\epsilon^{A B}\left(\frac{1}{4} \bar{D}_{A} \bar{D}_{C} C_{B}^{C}+\frac{1}{8} C_{A}^{C} N_{C B}\right) .
\end{aligned}
$$

The first column contains the charge aspects $\left(\Psi_{0 A B}, \Psi_{1 A}, \operatorname{Re} \Psi_{2}\right)$ that satisfy an evolution equation on $\mathcal{I}$. The second column contains the components of the Weyl tensor that provide information about the radiative nature of asymptotic infinity. Note that $\left(\Psi_{4}\right)_{A B}$ and $\left(\Psi_{0}\right)_{A B}$ are symmetric and tracefree tensors, $\left(\psi_{3}\right)_{A}$ and $\left(\psi_{1}\right)_{A}$ are vectors, while $\operatorname{Re} \psi_{2}$ and $\operatorname{Im} \psi_{2}$ are scalar quantities. Moreover, it is worth emphasizing that $\left(\Psi_{3}\right)_{A}$ is $M_{A}, \operatorname{Re} \Psi_{2}$ is the covariant mass $\mathcal{M}, \operatorname{Im} \Psi_{2}$ is the covariant dual mass $\tilde{\mathcal{M}}$, and $\left(\Psi_{1}\right)_{A}$ is the covariant momentum $\mathcal{P}_{A}$ introduced in section 4.5 to be those quantities that transform under the BMSW without any quadratic anomaly.

An explicit computation of the leading asymptotic values of the Weyl scalars shows that

$$
\begin{aligned}
\psi_{4} & :=W_{\hat{0} \hat{3} \hat{0} \hat{3}}=\frac{1}{r} \hat{\bar{E}}_{3}^{A} \hat{\bar{E}}_{3}^{B}\left(\Psi_{4}\right)_{A B}+o\left(r^{-1}\right), \\
\psi_{3} & :=W_{\hat{0} \hat{3} \hat{0} \hat{1}}=\frac{1}{r^{2}} \hat{\bar{E}}_{3}^{A}\left(\Psi_{3}\right)_{A}+o\left(r^{-2}\right), \\
\operatorname{Re} \psi_{2} & :=W_{\hat{1} \hat{0} \hat{1} \hat{0}}=\frac{1}{r^{3}} \operatorname{Re} \Psi_{2}+o\left(r^{-3}\right), \\
i \operatorname{Im} \psi_{2} & :=W_{\hat{1} \hat{0} \hat{2} \hat{3}}=\frac{1}{r^{3}} i \operatorname{Im} \Psi_{2}+o\left(r^{-3}\right), \\
\psi_{1} & :=W_{\hat{1} \hat{0} \hat{1} \hat{2}}=\frac{1}{r^{4}} \hat{\bar{E}}_{2}^{A}\left(\bar{\Psi}_{1}\right)_{A}+o\left(r^{-4}\right), \\
\psi_{0} & :=W_{\hat{1} \hat{2} \hat{1} \hat{2}}=\frac{1}{r^{5}} \hat{\bar{E}}_{2}^{A} \hat{\bar{E}}_{2}^{B}\left(\bar{\Psi}_{0}\right)_{A B}+o\left(r^{-5}\right) .
\end{aligned}
$$


Open Access. This article is distributed under the terms of the Creative Commons Attribution License (CC-BY 4.0), which permits any use, distribution and reproduction in any medium, provided the original author(s) and source are credited.

\section{References}

[1] E. Noether, Invariant variation problems, Gott. Nachr. 1918 (1918) 235 [physics/0503066] [INSPIRE].

[2] H. Bondi, Gravitational waves in general Relativity, Nature 186 (1960) 535 [INSPIRE].

[3] H. Bondi, M. G. J. van der Burg and A.W.K. Metzner, Gravitational waves in general relativity. 7. Waves from axisymmetric isolated systems, Proc. Roy. Soc. Lond. A 269 (1962) 21.

[4] R.K. Sachs, On the characteristic initial value problem in gravitational theory, J. Math. Phys. 3 (1962) 908 [InSPIRE].

[5] E.T. Newman and T.W.J. Unti, Behavior of asymptotically flat empty spaces, J. Math. Phys. 3 (1962) 891 [INSPIRE].

[6] T. Mädler and J. Winicour, Bondi-Sachs formalism, Scholarpedia 11 (2016) 33528 [arXiv: 1609.01731] [INSPIRE].

[7] S. Weinberg, Infrared photons and gravitons, Phys. Rev. 140 (1965) B516 [InSPIRE].

[8] D. Christodoulou, Nonlinear nature of gravitation and gravitational wave experiments, Phys. Rev. Lett. 67 (1991) 1486 [INSPIRE].

[9] K.S.T. Braginsky, Gravitational-wave bursts with memory and experimental prospects, Nature 327 (1987) 123.

[10] A. Strominger, On BMS invariance of gravitational scattering, JHEP 07 (2014) 152 [arXiv: 1312.2229] [INSPIRE].

[11] A. Strominger and A. Zhiboedov, Gravitational memory, BMS supertranslations and soft theorems, JHEP 01 (2016) 086 [arXiv:1411.5745] [INSPIRE].

[12] A. Strominger, Lectures on the infrared structure of gravity and gauge theory, arXiv: 1703.05448 [INSPIRE].

[13] G. Compère and A. Fiorucci, Advanced lectures on general relativity, arXiv:1801.07064 [INSPIRE].

[14] F. Cachazo and A. Strominger, Evidence for a new soft graviton theorem, arXiv:1404.4091 [INSPIRE].

[15] S. Pasterski, A. Strominger and A. Zhiboedov, New gravitational memories, JHEP 12 (2016) 053 [arXiv: 1502.06120] [INSPIRE].

[16] M. Campiglia and A. Laddha, Asymptotic symmetries and subleading soft graviton theorem, Phys. Rev. D 90 (2014) 124028 [arXiv:1408.2228] [InSPIRE].

[17] E.E. Flanagan and D.A. Nichols, Conserved charges of the extended Bondi-Metzner-Sachs algebra, Phys. Rev. D 95 (2017) 044002 [arXiv: 1510.03386] [INSPIRE].

[18] G. Compère, A. Fiorucci and R. Ruzziconi, Superboost transitions, refraction memory and super-Lorentz charge algebra, JHEP 11 (2018) 200 [Erratum ibid. 04 (2020) 172] [arXiv: 1810.00377] [INSPIRE]. 
[19] E. Himwich, Z. Mirzaiyan and S. Pasterski, A note on the subleading soft graviton, JHEP 04 (2021) 172 [arXiv: 1902.01840] [INSPIRE].

[20] G. Barnich and C. Troessaert, Symmetries of asymptotically flat 4 dimensional spacetimes at null infinity revisited, Phys. Rev. Lett. 105 (2010) 111103 [arXiv:0909.2617] [InSPIRE].

[21] G. Barnich and C. Troessaert, BMS charge algebra, JHEP 12 (2011) 105 [arXiv: 1106.0213] [INSPIRE].

[22] L. Donnay, S. Pasterski and A. Puhm, Asymptotic symmetries and celestial CFT, JHEP 09 (2020) 176 [arXiv: 2005.08990] [INSPIRE].

[23] D. Kapec, V. Lysov, S. Pasterski and A. Strominger, Semiclassical Virasoro symmetry of the quantum gravity $\mathcal{S}$-matrix, JHEP 08 (2014) 058 [arXiv:1406.3312] [INSPIRE].

[24] D. Kapec, P. Mitra, A.-M. Raclariu and A. Strominger, $2 D$ stress tensor for $4 D$ gravity, Phys. Rev. Lett. 119 (2017) 121601 [arXiv:1609.00282] [INSPIRE].

[25] S. Pasterski, S.-H. Shao and A. Strominger, Flat space amplitudes and conformal symmetry of the celestial sphere, Phys. Rev. D 96 (2017) 065026 [arXiv:1701.00049] [InSPIRE].

[26] L. Donnay, G. Giribet, H.A. Gonzalez and M. Pino, Supertranslations and Superrotations at the black hole horizon, Phys. Rev. Lett. 116 (2016) 091101 [arXiv:1511. 08687] [INSPIRE].

[27] L. Donnay, G. Giribet, H.A. González and M. Pino, Extended symmetries at the black hole horizon, JHEP 09 (2016) 100 [arXiv: 1607.05703] [INSPIRE].

[28] L. Donnay and C. Marteau, Carrollian physics at the black hole horizon, Class. Quant. Grav. 36 (2019) 165002 [arXiv: 1903.09654] [INSPIRE].

[29] F. Hopfmüller and L. Freidel, Gravity degrees of freedom on a null surface, Phys. Rev. D 95 (2017) 104006 [arXiv:1611.03096] [inSPIRE].

[30] W. Wieland, New boundary variables for classical and quantum gravity on a null surface, Class. Quant. Grav. 34 (2017) 215008 [arXiv: 1704.07391] [INSPIRE].

[31] V. Chandrasekaran, E.E. Flanagan and K. Prabhu, Symmetries and charges of general relativity at null boundaries, JHEP 11 (2018) 125 [arXiv: 1807.11499] [INSPIRE].

[32] W. Wieland, Null infinity as an open Hamiltonian system, JHEP 04 (2021) 095 [arXiv: 2012.01889] [INSPIRE].

[33] W. Donnelly and L. Freidel, Local subsystems in gauge theory and gravity, JHEP 09 (2016) 102 [arXiv: 1601.04744$]$ [INSPIRE].

[34] L. Freidel and A. Perez, Quantum gravity at the corner, Universe 4 (2018) 107 [arXiv: 1507.02573] [INSPIRE].

[35] L. Freidel, A. Perez and D. Pranzetti, Loop gravity string, Phys. Rev. D 95 (2017) 106002 [arXiv: 1611.03668] [INSPIRE].

[36] L. Freidel and E.R. Livine, Bubble networks: framed discrete geometry for quantum gravity, Gen. Rel. Grav. 51 (2019) 9 [arXiv:1810.09364] [inSPIRE].

[37] L. Freidel, E.R. Livine and D. Pranzetti, Gravitational edge modes: from Kac-Moody charges to Poincaré networks, Class. Quant. Grav. 36 (2019) 195014 [arXiv:1906.07876] [INSPIRE].

[38] L. Freidel, M. Geiller and D. Pranzetti, Edge modes of gravity. Part I. Corner potentials and charges, JHEP 11 (2020) 026 [arXiv:2006.12527] [INSPIRE]. 
[39] L. Freidel, M. Geiller and D. Pranzetti, Edge modes of gravity. Part II. Corner metric and Lorentz charges, JHEP 11 (2020) 027 [arXiv: 2007.03563] [INSPIRE].

[40] L. Freidel, M. Geiller and D. Pranzetti, Edge modes of gravity. Part III. Corner simplicity constraints, JHEP 01 (2021) 100 [arXiv:2007.12635] [INSPIRE].

[41] W. Donnelly, L. Freidel, S.F. Moosavian and A.J. Speranza, Gravitational edge modes, coadjoint orbits, and hydrodynamics, arXiv:2012.10367 [INSPIRE].

[42] E.R. Livine, Loop quantum gravity boundary dynamics and gauge theory, Class. Quant. Grav. 38 (2021) 135031 [arXiv:2101.07565] [INSPIRE].

[43] Q. Chen and E.R. Livine, Loop quantum gravity's boundary maps, arXiv:2103.08409 [INSPIRE].

[44] L. Freidel, R. Oliveri, D. Pranzetti and S. Speziale, Extended corner symmetry, charge bracket and Einstein's equations, arXiv:2104.12881 [INSPIRE].

[45] C. Troessaert, Hamiltonian surface charges using external sources, J. Math. Phys. 57 (2016) 053507 [arXiv: 1509.09094] [INSPIRE].

[46] R.M. Wald and A. Zoupas, A general definition of 'conserved quantities' in general relativity and other theories of gravity, Phys. Rev. D 61 (2000) 084027 [gr-qc/9911095] [INSPIRE].

[47] V. Chandrasekaran and A.J. Speranza, Anomalies in gravitational charge algebras of null boundaries and black hole entropy, JHEP 01 (2021) 137 [arXiv:2009.10739] [INSPIRE].

[48] G. Barnich and C. Troessaert, Aspects of the BMS/CFT correspondence, JHEP 05 (2010) 062 [arXiv: 1001.1541] [INSPIRE].

[49] G. Barnich and G. Compere, Surface charge algebra in gauge theories and thermodynamic integrability, J. Math. Phys. 49 (2008) 042901 [arXiv:0708.2378] [InSPIRE].

[50] G. Barnich and C. Troessaert, Comments on holographic current algebras and asymptotically flat four dimensional spacetimes at null infinity, JHEP 11 (2013) 003 [arXiv: 1309.0794] [INSPIRE].

[51] G. Compère, A. Fiorucci and R. Ruzziconi, The $\Lambda$-BMS 4 charge algebra, JHEP 10 (2020) 205 [arXiv: 2004.10769] [inSPIRE].

[52] H. Adami, M.M. Sheikh-Jabbari, V. Taghiloo, H. Yavartanoo and C. Zwikel, Symmetries at null boundaries: two and three dimensional gravity cases, JHEP 10 (2020) 107 [arXiv: 2007.12759] [INSPIRE].

[53] R. Ruzziconi and C. Zwikel, Conservation and integrability in lower-dimensional gravity, JHEP 04 (2021) 034 [arXiv:2012.03961] [INSPIRE].

[54] F. Alessio, G. Barnich, L. Ciambelli, P. Mao and R. Ruzziconi, Weyl charges in asymptotically locally AdS s spacetimes, Phys. Rev. D 103 (2021) 046003 [arXiv: 2010.15452] [INSPIRE].

[55] A. Fiorucci and R. Ruzziconi, Charge algebra in Al(A)dS spacetimes, JHEP 05 (2021) 210 [arXiv: 2011.02002] [INSPIRE].

[56] G. Compère, R. Oliveri and A. Seraj, The Poincaré and BMS flux-balance laws with application to binary systems, JHEP 10 (2020) 116 [arXiv:1912.03164] [INSPIRE].

[57] J. D. Kijowski and W. Szczyrba, A canonical structure for classical field theories, Commun. Math. Phys. 46 (1976) 183. 
[58] K. Gawȩdzki, Classical origin of quantum group symmetries in Wess-Zumino-Witten conformal field theory, Commun. Math. Phys. 139 (1991) 201.

[59] C. Crnkovic and E. Witten, Covariant description of canonical formalism in geometrical theories, in Three hundred years of gravitation, S.W. Hawking and W. Israel., Cambridge University Press, Cambridge U.K. (1987), p. 676.

[60] A. Ashtekar, L. Bombelli and O. Reula, The covariant phase space of asymptotically flat gravitational fields, in Mechanics, analysis and geometry: 200 years after lagrange, M. Francaviglia ed., North-Holland Delta Series, hrefhttp://dx.doi.org/10.1016/B978-0-444-88958-4.50021-5Elsevier, Amsterdam The Netherlands (1991), p. 417.

[61] J. Lee and R.M. Wald, Local symmetries and constraints, J. Math. Phys. 31 (1990) 725 [INSPIRE].

[62] M. Campiglia and J. Peraza, Generalized BMS charge algebra, Phys. Rev. D 101 (2020) 104039 [arXiv:2002.06691] [INSPIRE].

[63] L. Ciambelli and R.G. Leigh, Isolated surfaces and symmetries of gravity, arXiv:2104.07643 [INSPIRE].

[64] B. Bonga and E. Poisson, Coulombic contribution to angular momentum flux in general relativity, Phys. Rev. D 99 (2019) 064024 [arXiv: 1808.01288] [INSPIRE].

[65] A. Ashtekar, T. De Lorenzo and N. Khera, Compact binary coalescences: The subtle issue of angular momentum, Phys. Rev. D 101 (2020) 044005 [arXiv:1910.02907] [INSPIRE].

[66] A. Elhashash and D.A. Nichols, Definitions of angular momentum and super angular momentum in asymptotically flat spacetimes: properties and applications to compact-binary mergers, Phys. Rev. D 104 (2021) 024020 [arXiv:2101.12228] [INSPIRE].

[67] P.-N. Chen, M.-T. Wang, Y.-K. Wang and S.-T. Yau, Supertranslation invariance of angular momentum, arXiv:2102.03235 [INSPIRE].

[68] G. Compère and D.A. Nichols, Classical and quantized general-relativistic angular momentum, arXiv:2103.17103 [INSPIRE].

[69] G. Compère and J. Long, Vacua of the gravitational field, JHEP 07 (2016) 137 [arXiv: 1601.04958] [INSPIRE].

[70] A. Ashtekar, Asymptotic quantization of the gravitational field, Phys. Rev. Lett. 46 (1981) 573 [INSPIRE].

[71] A. Ashtekar and M. Streubel, Symplectic geometry of radiative modes and conserved quantities at null infinity, Proc. Roy. Soc. Lond. A 376 (1981) 585.

[72] L. Ciambelli, C. Marteau, A.C. Petkou, P.M. Petropoulos and K. Siampos, Flat holography and Carrollian fluids, JHEP 07 (2018) 165 [arXiv: 1802.06809] [INSPIRE].

[73] L. Ciambelli, R.G. Leigh, C. Marteau and P.M. Petropoulos, Carroll structures, null geometry and conformal isometries, Phys. Rev. D 100 (2019) 046010 [arXiv: 1905.02221] [INSPIRE].

[74] Y. Herfray, Asymptotic shear and the intrinsic conformal geometry of null-infinity, J. Math. Phys. 61 (2020) 072502 [arXiv:2001.01281] [INSPIRE].

[75] Y. Herfray, Tractor geometry of asymptotically flat space-times, arXiv:2103.10405 [INSPIRE]. 
[76] M.P. Reisenberger, The Poisson bracket on free null initial data for gravity, Phys. Rev. Lett. 101 (2008) 211101 [arXiv:0712.2541] [INSPIRE].

[77] M.P. Reisenberger, The symplectic 2-form for gravity in terms of free null initial data, Class. Quant. Grav. 30 (2013) 155022 [arXiv:1211.3880] [INSPIRE].

[78] K. Parattu, S. Chakraborty, B.R. Majhi and T. Padmanabhan, A boundary term for the gravitational action with null boundaries, Gen. Rel. Grav. 48 (2016) 94 [arXiv: 1501.01053] [INSPIRE].

[79] M.P. Reisenberger, The Poisson brackets of free null initial data for vacuum general relativity, Class. Quant. Grav. 35 (2018) 185012 [arXiv:1804.10284] [INSPIRE].

[80] F. Hopfmüller and L. Freidel, Null conservation laws for gravity, Phys. Rev. D 97 (2018) 124029 [arXiv: 1802.06135] [INSPIRE].

[81] R. Oliveri and S. Speziale, Boundary effects in general relativity with tetrad variables, Gen. Rel. Grav. 52 (2020) 83 [arXiv: 1912.01016] [INSPIRE].

[82] M. Campiglia and A. Laddha, New symmetries for the gravitational S-matrix, JHEP 04 (2015) 076 [arXiv: 1502.02318] [INSPIRE].

[83] L. Freidel, E.R. Livine and D. Pranzetti, Kinematical gravitational charge algebra, Phys. Rev. D 101 (2020) 024012 [arXiv: 1910.05642] [INSPIRE].

[84] R.F. Penna, BMS invariance and the membrane paradigm, JHEP 03 (2016) 023 [arXiv:1508.06577] [INSPIRE].

[85] R.F. Penna, Near-horizon BMS symmetries as fluid symmetries, JHEP 10 (2017) 049 [arXiv: 1703. 07382] [INSPIRE].

[86] I. M. Anderson, Introduction to the variational bicomplex, Contemp. Math. 132 (1992) 51.

[87] J. François, Bundle geometry of the connection space, covariant Hamiltonian formalism, the problem of boundaries in gauge theories, and the dressing field method, JHEP 03 (2021) 225 [arXiv: 2010.01597] [INSPIRE].

[88] R.K. Sachs, Gravitational waves in general relativity. 8. Waves in asymptotically flat space-times, Proc. Roy. Soc. Lond. A 270 (1962) 103.

[89] H. Bondi, M.G.J. van der Burg and A.W.K. Metzner, Gravitational waves in general relativity. 7. Waves from axisymmetric isolated systems, Proc. Roy. Soc. Lond. A 269 (1962) 21 [INSPIRE].

[90] G. Barnich and C. Troessaert, Finite BMS transformations, JHEP 03 (2016) 167 [arXiv: 1601.04090] [INSPIRE].

[91] L. Blanchet, Radiative gravitational fields in general relativity II. Asymptotic behaviour at future null infinity, Proc. Roy. Soc. London A 409 (1987) 383.

[92] L. Blanchet, G. Compère, G. Faye, R. Oliveri and A. Seraj, Multipole expansion of gravitational waves: from harmonic to Bondi coordinates, JHEP 02 (2021) 029 [arXiv:2011.10000] [INSPIRE].

[93] S. de Haro, S.N. Solodukhin and K. Skenderis, Holographic reconstruction of space-time and renormalization in the AdS/CFT correspondence, Commun. Math. Phys. 217 (2001) 595 [hep-th/0002230] [INSPIRE].

[94] I. Papadimitriou and K. Skenderis, Thermodynamics of asymptotically locally AdS spacetimes, JHEP 08 (2005) 004 [hep-th/0505190] [INSPIRE]. 
[95] G. Compere and D. Marolf, Setting the boundary free in AdS/CFT, Class. Quant. Grav. 25 (2008) 195014 [arXiv:0805.1902] [INSPIRE].

[96] L. Freidel, F. Hopfmüller and A. Riello, Asymptotic renormalization in flat space: symplectic potential and charges of electromagnetism, JHEP 10 (2019) 126 [arXiv: 1904.04384] [INSPIRE].

[97] A. Ashtekar, L. Bombelli and O. Reula, The covariant phase space of asymptotically flat gravitational fields, in Analysis, geometry and mechanics: 200 years after Lagrange. M. Francaviglia and D. Holm eds., North-Holland, The Netherlands (1991)-

[98] G. Barnich and F. Brandt, Covariant theory of asymptotic symmetries, conservation laws and central charges, Nucl. Phys. B 633 (2002) 3 [hep-th/0111246] [inSPIRE].

[99] M. Rangamani, Gravity and hydrodynamics: lectures on the fluid-gravity correspondence, Class. Quant. Grav. 26 (2009) 224003.

[100] R. Ruzziconi, On the Various Extensions of the BMS Group, Ph.D. thesis, Université libre de Bruxelles, Bruxelles (2020), arXiv:2009.01926 [InSPIRE].

[101] G. Barnich and P.H. Lambert, A note on the newman-unti group and the bms charge algebra in terms of Newman-Penrose coefficients, Adv. Math. Phys. 2012 (2012) 1.

[102] L. Ciambelli and R.G. Leigh, Weyl connections and their role in holography, Phys. Rev. D 101 (2020) 086020 [arXiv: 1905.04339] [INSPIRE].

[103] G. Barnich, P. Mao and R. Ruzziconi, BMS current algebra in the context of the Newman-Penrose formalism, Class. Quant. Grav. 37 (2020) 095010 [arXiv:1910.14588] [INSPIRE].

[104] L. Freidel and D. Pranzetti, Gravity from symmetry, to appear (2021).

[105] S.W. Hawking, M.J. Perry and A. Strominger, Superrotation charge and supertranslation hair on black holes, JHEP 05 (2017) 161 [arXiv:1611.09175] [INSPIRE].

[106] G. Barnich and R. Ruzziconi, Coadjoint representation of the BMS group on celestial Riemann surfaces, JHEP 06 (2021) 079 [arXiv:2103.11253] [inSPIRE].

[107] A. Ashtekar, J. Engle and D. Sloan, Asymptotics and Hamiltonians in a first order formalism, Class. Quant. Grav. 25 (2008) 095020 [arXiv:0802.2527] [INSPIRE].

[108] A. Ashtekar and B. Krishnan, Isolated and dynamical horizons and their applications, Living Rev. Rel. 7 (2004) 10 [gr-qc/0407042] [INSPIRE].

[109] J. Díaz-Polo and D. Pranzetti, Isolated horizons and black hole entropy in loop quantum gravity, SIGMA 8 (2012) 048 [arXiv: 1112.0291] [INSPIRE].

[110] H. Godazgar, M. Godazgar and C.N. Pope, New dual gravitational charges, Phys. Rev. D 99 (2019) 024013 [arXiv: 1812.01641] [INSPIRE].

[111] H. Godazgar, M. Godazgar and M.J. Perry, Hamiltonian derivation of dual gravitational charges, JHEP 09 (2020) 084 [arXiv:2007.07144] [INSPIRE].

[112] R. Oliveri and S. Speziale, A note on dual gravitational charges, JHEP 12 (2020) 079 [arXiv: 2010.01111] [INSPIRE].

[113] E. De Paoli and S. Speziale, A gauge-invariant symplectic potential for tetrad general relativity, JHEP 07 (2018) 040 [arXiv:1804.09685] [INSPIRE]. 
[114] T. Jacobson and A. Mohd, Black hole entropy and Lorentz-diffeomorphism Noether charge, Phys. Rev. D 92 (2015) 124010 [arXiv:1507.01054] [InSPIRE].

[115] K. Prabhu, The first law of black hole mechanics for fields with internal gauge freedom, Class. Quant. Grav. 34 (2017) 035011 [arXiv: 1511.00388] [INSPIRE].

[116] G. Barnich, P. Mao and R. Ruzziconi, Conserved currents in the Cartan formulation of general relativity, in About Various Kinds of Interactions: Workshop in honour of ProfeSSOR Philippe Spindel,June 4-5, Mons, Belgium (2015), arXiv:1611.01777 [INSPIRE].

[117] E. Frodden and D. Hidalgo, Surface charges for gravity and electromagnetism in the first order formalism, Class. Quant. Grav. 35 (2018) 035002 [arXiv:1703.10120] [INSPIRE].

[118] H. Gomes and A. Riello, Unified geometric framework for boundary charges and particle dressings, Phys. Rev. D 98 (2018) 025013 [arXiv:1804.01919] [InSPIRE].

[119] J. Margalef-Bentabol and E.J.S. Villaseñor, Geometric formulation of the covariant phase space methods with boundaries, Phys. Rev. D 103 (2021) 025011 [arXiv:2008.01842] [INSPIRE].

[120] J.F.B. G., J. Margalef-Bentabol, V. Varo and E.J.S. Villaseñor, Covariant phase space for gravity with boundaries: metric vs tetrad formulations, arXiv:2103.06362 [INSPIRE].

[121] E. De Paoli and S. Speziale, Sachs' free data in real connection variables, JHEP 11 (2017) 205 [arXiv: 1707.00667] [inSPIRE].

[122] H. Godazgar, M. Godazgar and M.J. Perry, Asymptotic gravitational charges, Phys. Rev. Lett. 125 (2020) 101301 [arXiv: 2007.01257] [INSPIRE].

[123] G. Compère, Infinite towers of supertranslation and superrotation memories, Phys. Rev. Lett. 123 (2019) 021101 [arXiv: 1904.00280] [INSPIRE]. 